\title{
Torsion-Free Abelian Group Rings III
}

\author{
Ryûki Matsuda*
}

In this paper a ring is a commutative ring with identity. Let $X$ be an indeterminate, $G$ a non-zero torsion-free abelian group (additively written). We consider the group ring $A_{G}[X]$ of $G$ over a ring $A . A_{G}[X]$ is the ring of elements $\sum a_{\alpha} X^{\alpha}, a_{\alpha} \in A, \alpha \in G$ almost all $a_{\alpha}$ are zero. This paper is a continuation of [26]. Let $I$ be any nonempty set, and $\left\{X_{i} ; I\right\}$ a set of indeterminates with the index set of $J$. We may define the group ring $A_{G}\left[X_{i} ; I\right]$ of $G$ over a ring $A$ with the indeterminates $\left\{X_{i} ; I_{j}\right.$. This paper consists of 14 sections. $\S 1$ is on the integral (elements), $\S 2$ is on the Kronecker functions rings, $\S 3$ is on the noetherian, $\S 4$ is on the pseudo-prüfer, $\S 5$ is on the extension $K_{G}[X] / k_{G}[X]$ for fields $K / k, \S 6$ is on the Hilbert, $\S 7$ is on $A_{G}[X]_{s}$ where $S$ is the monics for a totally ordered $G$, $\S 8$ is on the factorial, $\S 9$ is on the chain conditions, $\S 10$ is on the finite characters, $\S 11$ is on the regularly noetherian, $\S 12$ is on the Dedekind, $\$ 13$ is on the rational functions rings, $\S 14$ is on the others. Throughout the paper, $A$ is a ring, $X$ an indeterminate, $G$ a non-zero torsion-free abelian group (additively written), $\left\{X_{i} ; I\right\}$ a set of indeterminates, $D$ an integral domain, $K$ the quotient field of $D, A_{G}\left(X_{i} ; I\right)$ the total quotient ring of $A_{G}\left[X_{i} ; I\right]$. Definitions of terms are those of [26]. We note that $G$ becomes a totally ordered abelian group.

\section{§1. The integral}

Let $\mathfrak{p}^{*}(D)$ be the ordered group of nonzero principal fractional ideals of an integral domain $D$ (cf. [6]).

Proposition 1. Let $D$ be a regularly integrally closed domain, $G_{f}(D)$ the ordered group generated by divisors of $D$ of finite type. Then, $\mathfrak{p}^{*}\left(D_{G}[X]\right)$ is canonically order-isomorphic onto a subgroup of the product ordered group $\mathfrak{p}^{*}\left(K_{G}[X]\right) \times G_{f}(D)$.

Proof. $K$ is the quotient field of $D$. We consider a mapping: $(f / g) D_{G}[X]$ $\mapsto\left((f / g) K_{G}[X], \operatorname{div} A_{f}-\operatorname{div} A_{g}\right)$. That this is a well-defined mapping and a homomorphism follows from [26] $\S 6$, Prop. 1 .

A ring $A$ is said integrally closed if $A$ is integrally closed in the total quotient

Received December 20, 1976.

* Department of Mathematics, Faculty of Science, Ibaraki University, Mito, Japan. 
ring.

Proposition 2. Assume every non-zerodivisor of $A$ is a unit. If $A_{G}[X]$ is integrally closed, $A$ is von Neumann regular.

Proof. Suppose $A$ has a nonzero nilpotent $a . a^{n}=0$ for some $n>0$. Take $0 \neq \alpha \in G . \quad a /(1+X)^{\alpha} \bar{\in} A_{G}[X] . \quad\left(a /\left(1+X^{\alpha}\right)\right)^{n}=0 . \quad A_{G}[X]$ is not integrally closed.

Proposition 3. If $A_{G}[X]$ is integrally closed, then $A$ is integrally closed and the nilradical of $A$ is zero.

Proposition 4. Let $B$ be the total quotient ring of a ring $A . A_{G}[X]$ is integrally closed if and only if $A$ is integrally closed and $B_{G}[X]$ is integrally closed.

Proof. We use [26] $\$ 6$, Th. 6.

Proposition 5. If $A$ is von Neumann regular, then $A_{2}\left[X_{1}, \ldots, X_{n}\right]$ is integrally closed.

Proof. By induction on $n$. Suppose $n=1$ and put $X_{1}=X$. Let $f / g \in A_{Z}(X)$ be integral over $A_{z}[X]$. We may take $f, g \in A[X]$. By [16](3.2), $A[X]$ is bezout. There exist $h, f^{\prime}, g^{\prime}, f_{1}, g_{1} \in A[X]$ such that $f=h f^{\prime}, g=h g^{\prime}, h=f f_{1}+g g_{1}$. It follows $1=f^{\prime} f_{1}+g^{\prime} g_{1}, 1 / g^{\prime}=(f / g) f_{1}+g_{1} .1 / g^{\prime}$ is integral over $A_{z}[X]$. It follows $1 / g^{\prime} \in A_{z}[X], f / g \in A_{z}[X]$. Suppose the proposition is OK till $n-1$. If $\xi \in A_{Z}\left(X_{1}, \ldots, X_{n}\right)$ is integral over $A_{Z}\left[X_{1}, \ldots, X_{n}\right]$, it follows $\xi \in\left(A_{Z}\left(X_{1}, \ldots\right.\right.$, $\left.\left.X_{n-1}\right)\right)_{Z}\left[X_{n}\right]$. By $[26] \S 6$, Th. 6, we have $\xi \in\left(A_{Z}\left[X_{1}, \ldots, X_{n-1}\right]\right)_{Z}\left[X_{n}\right]$.

Proposition 6. If $A$ is von Neumann regular, $A_{G}[X]$ is integrally closed.

Proof. Let $f / g \in A_{G}(X)$ be integral over $A_{G}[X]$.

$$
(f / g)^{n}+(f / g)^{n-1} h_{n-1}+\cdots+h_{0}=0
$$

for $h_{i} \in A_{G}[X]$. Let $H$ be the subgroup of $G$ generated by the powers of $f, g, h_{n-1}$, $\ldots, h_{0} . f / g \in A_{H}(X)$ is integral over $A_{H}[X]$. By Prop. 5, we have $f / g \in A_{H}[X]$.

THEOREM 7. $A_{G}[X]$ is integrally closed if and only if $A$ is integrally closed and of zero nilradical.

Proof. The necessity follows from Prop. 3. The sufficiency. The total quotient ring $B$ of $A$ is von Neumann regular. By Prop. 6, $B_{G}[X]$ is integrally closed. By Prop. 4, $A_{G}[X]$ is integrally closed.

Lemma 8. Let $\mathfrak{a}, \mathfrak{b}$ be fractional ideals $\neq 0$ of $a$ domain $D . \quad$ If $\operatorname{div} \mathfrak{a}=\operatorname{div} \mathfrak{b}$ on $D$, then Div $\mathfrak{a} D_{G}[X]=\operatorname{Div} \mathfrak{b} D_{G}[X]$ on $D_{G}[X]$.

Proof. Assume $\xi \in D_{G}(X), a D_{G}[X] \subset \xi D_{G}[X]$. $\xi=a / g$ for $a \in D, g \in D_{G}[X]$. 
We set $g=\sum b_{\alpha} X^{\alpha} . \quad \mathfrak{a} \subset\left(a / b_{\alpha}\right) D . \quad \mathfrak{b} \subset\left(a / b_{\alpha}\right) D . \quad \mathfrak{b} \subset(a / g) D_{G}[X] . \quad \mathfrak{b} D_{G}[X] \subset \xi D_{G}[X]$. Div $a D_{G}[X] \leq \operatorname{Div} b D_{G}[X]$ on $D_{G}[X]$. Similarly, Div $\mathfrak{a} D_{G}[X] \geq \operatorname{Div} \mathfrak{b} D_{G}[X]$. Hence, Div $a D_{G}[X]=\operatorname{Div} b D_{G}[X]$.

THEOREM 9. $D_{G}[X]$ is regularly integrally closed if and only if $D$ is regularly integrally closed.

Proof. The sufficiency is over in [26] $\S 6$, Th. 4. The necessity. Assume $\operatorname{div} \mathfrak{a}+\operatorname{div}\left(x_{1}, \ldots, x_{n}\right)=\operatorname{div} \mathrm{b}+\operatorname{div}\left(x_{1}, \ldots, x_{n}\right)$ on $D$ for $x_{i} \in K$. $\operatorname{div} \mathrm{a}\left(x_{1}, \ldots, x_{n}\right)=$ $\operatorname{div} \mathfrak{b}\left(x_{1}, \ldots, x_{n}\right)$. By Lem. 8, $\operatorname{Div} \mathfrak{a}\left(x_{1}, \ldots, x_{n}\right) D_{G}[X]=\operatorname{Divb}\left(x_{1}, \ldots, x_{n}\right) D_{G}[X]$ on $D_{G}[X]$. $\quad \operatorname{Div} a D_{G}[X]+\operatorname{Div}\left(x_{1}, \ldots, x_{n}\right)=\operatorname{Div} b D_{G}[X]+\operatorname{Div}\left(x_{1}, \ldots, x_{n}\right)$. Hence, $\operatorname{Div} \mathfrak{a} D_{G}[X]=\operatorname{Div} \mathfrak{b} D_{G}[X]$. Assume $\mathfrak{a} \subset x D, x \in K . \quad \mathfrak{a} D_{G}[X] \subset x D_{G}[X] . \quad \mathfrak{b} D_{G}[X]$ $\subset x D_{G}[X] . \quad \mathfrak{b} \subset x D$. Hence, $\operatorname{div} \mathfrak{a} \leq \operatorname{div} \mathfrak{b}$. Similarly, $\operatorname{div} \mathfrak{a} \geq \operatorname{div} \mathfrak{b}$. Hence, $\operatorname{div} a$ $=\operatorname{div} b$.

Let $B$ be the total quotient ring of $A$. If every $x \in B$ which is almost integral over $A$ belongs to $A, A$ is said completely integrally closed ([13] p. 132).

Proposition 10. If $A_{G}[X]$ is completely integrally closed, then $A$ is completely integrally closed.

Lemma 11. Let $A$ be a von Neumann regular ring, $g$ a non-zerodivisor of $A[X]$. If $1 / g$ is almost integral over $A[X]$, then $1 / g \in A[X]$.

Proof. By the induction on $\operatorname{deg} g=n$. Suppose the proposition is $O K$ till $n-1$. Let $b$ be the coefficient of the highest degree of $g . \quad b A=e A, e^{2}=e$ for $e \in A . \quad A=e A \oplus(1-e) A . \quad g=e g+(1-e) g$. Since $(1-e) /((1-e) g) \quad$ is almost integral over $(1-e) A[X]$ and $\operatorname{deg}(1-e) g$ is less than $n$, we have $(1-e) /((1-e) g)$ $\in(1-e) A[X]$. Since $e /(e g)$ is almost integral over $e . A[X]$ and $e g$ is a monic of $e A[X]$, it follows $e /(e g) \in e A[X]$. Hence $1 / g \in A[X]$.

Lemma 12. Assume $A$ is the total quotient ring of itself. Then $A[X]$ is completely integrally closed if and only if $A$ is von Neumann regular.

Proof. The necessity. The nilradical of $A$ is zero. The sufficiency. Similar with the proof of Prop. 5, using Lem. 11.

Lemma 13. Assume $A$ is von Neumann regular. If $f / g \in A_{Z}(X)$ is almost integral over $A_{Z}[X]$, then $f / g \in A_{Z}[X]$.

Proof. We may take $f, g \in A[X]$. Set $g=a_{0}+a_{1} X+\cdots+a_{n} X^{n}$. We prove the proposition by the induction on $\operatorname{deg} g=n$. Suppose the proposition is OK till $n-1$. If $a_{0}=0, f /\left(a_{1}+\cdots+a_{n} X^{n-1}\right)$ is almost integral over $A_{Z}[X]$; hence $\in A_{Z}[X]$; hence $f / g \in A_{Z}[X]$. Hence, we may suppose $a_{0} \neq 0$. $a_{0} A=e A$, $e^{2}=e$ for $e \in A . \quad((1-e) f) /((1-e) g)$ is almost integral over $(1-e) A_{Z}[X]$. $((1-e) f) /\left((1-e)\left(a_{1}+\cdots+a_{n} X^{n-1}\right)\right)$ is almost integral over $(1-e) A_{z}[X]$; 
hence $\epsilon(1-e) A_{z}[X]$; hence $((1-e) f) /((1-e) g) \in(1-e) A_{z}[X] . \quad(e f) /(e g) \quad$ is almost integral over $e A_{Z}[X]$. There exists non-zerodivisor $g_{1}$ of $e A[X]$ such that $g_{1}((e f) /(e g))^{m}=H_{m} \in e A_{z}[X]$. Then, $H_{m} \in e A[X]$. By Lem. 12, it follows $(e f) /(e g) \in e A[X]$. Hence, $f / g \in A_{Z}[X]$.

Lemma 14. Assume $A$ is its own total quotient ring. Then $A_{z}\left[X_{1}, \ldots, X_{n}\right]$ is completely integrally closed if and only if $A$ is von Neumann regular.

Proof. The necessity. By Prop. 3, the nilradical of $A$ is zero. The sufficiency. By the induction on $n$. By Lem. 13, the proposition is OK for $n=1$. Suppose we are over till $n-1$. Assume $\xi \in A_{Z}\left(X_{1}, \ldots, X_{n}\right)$ is almost integral over $A_{z}\left[X_{1}, \ldots, X_{n}\right]$. By Lem. 13, it follows $\xi \in A_{Z}\left(X_{1}, \ldots, X_{n-1}\right)_{z}\left[X_{n}\right]$. By the hypothesis and $[26] \S 6, T h .8$, it follows $\zeta \in A_{Z}\left[X_{1}, \ldots, X_{n}\right]$.

Proposition 15. Assume $A$ is its own total quotient ring. Then $A_{G}[X]$ is completely integrally closed if and only' if $A$ is von Neumann regular.

Proof. The sufficiency. Assume $f / g \in A_{G}(X)$ be almost integral over $A_{G}[X]$. There exists a non-zerodivisor $h$ of $A_{G}[X]$ such that $h(f / g)^{n}=h_{n} \in A_{G}[X]$. Let $H$ be the subgroup of $G$ generated by the powers of $h, f, g$ and let $K$ be the subgroup of $G$ generated by the powers of $h, f, g, h_{n} . \quad K=Z \alpha_{1} \oplus \cdots \oplus Z \alpha_{m}, H=Z e_{1} \alpha_{1}$ $\oplus \cdots \oplus Z e_{k} \alpha_{k}, 0<e_{i}, e_{i} \mid e_{i+1}$. Then, $k=m . \quad h_{n}$ is integral over $A_{H}[X]$. By Prop. 6, it follows $h_{n} \in A_{H}[X] . \quad f / g \in A_{H}(X)$ is almost integral over $A_{H}[X]$. By Lem. 14, it follows $f / g \in A_{H}[X]$. Hence, $A_{G}[X]$ is completely integrally closed.

THEOREM 16. $A_{G}[X]$ is completely integrally closed if and only if $A$ is completely integrally closed and the nilradical of $A$ is zero.

Proof. The sufficiency. By Prop. $15, B_{G}[X]$ is completely integrally closed, where $B$ is the total quotient ring of $A$. Assume $\xi \in A_{G}(X)$ be almost integral over $A_{G}[X]$. Then, $\xi \in B_{G}[X]$. By [26] 8 , Th. 8, it follows $\xi \in A_{G}[X]$.

The next is on the japanese rings [20] (23.1.1).

Proposition 17. If $D_{G}[X]$ is japanese, then $D$ is japanese.

Proof. Assume $L$ be a finite field extension of $K$. By [24] (4.4), the integral closure of $D_{G}[X]$ in $L_{G}(X)$ is the integral closure of $D_{G}[X]$ in $L_{G}[X]$. Let $I$ be the integral closure of $D$ in $L$. By [26] $\$ 6$, Th. 6, the integral closure of $D_{G}[X]$ in $L_{G}[X]$ is $I_{G}[X]$. $I_{G}[X]=\sum_{1}^{n} D_{G}[X] F_{i}$ for $F_{i} \in I_{G}[X]$. We set $F_{i}=\sum b_{i \alpha} X^{\alpha}$. Then, $I=\sum_{i, \alpha} D b_{i \alpha}$.

RemarK 18. If $D$ is a japanese ring, then $D_{Z}[X]$ is a japanese ring.

Proof. By $[30](9.0 .2), D[X]$ is japanese. Hence, $D_{Z}[X]$ is japanese. 
A noetherian ring $A$ is said $D$-ring if all of its overrings are integrally closed [10]. A subring of the total quotient ring of $A$ containing $A$ is a overring of $A$.

Proposition 19. If $A_{G}[X]$ is a D-ring, then $A$ is a D-ring.

Proof. Let $B$ be an overring of $A$. Then, $B_{G}[X]$ is an overring of $A_{G}[X]$. Hence, $B_{G}[X]$ is integrally closed. By Prop. $3, B$ is integrally closed. Hence, $A$ is a $D$-ring.

Theorem 20. $D_{G}[X]$ is a $D$-domain if and only if $D$ is a ficld and $G \cong Z$.

Proof. The necessity. $D_{G}[X]$ is integrally closed. By $[10]$ Th. $1, D_{G}[X]$ is a $C$-domain. By $\S 3$, Th. $7, D$ is a field and $G \cong Z$. The sufficiency. $D_{Z}[X]$ has the QR-property. Hence, overrings of $D_{z}[X]$ are integrally closed. Hence, $D_{Z}[X]$ is a $D$-domain.

Theorem 21. $A_{G}[X]$ is a D-ring if and only if $A_{G}[X]$ is a C-ring.

Proof. [10] Th. 1 and $\$ 3$, Th. 7.

The next is on the universally japanese rings $[20](0,23.1 .1)$.

Proposition 22. If $A_{\mathrm{G}}[X]$ is universally japanese, then $A$ is universally japanese.

Proof. An $A$-algebra of finite type is an $A_{G}[X]$-algebra of finite type.

Lemma 23. Let $S$ be a ring, $R$ a subring of $S$ such that $R$ is integrally closed in $S, G=Z \oplus Z$ with the lexicographic order. Let $H$ be a monic clement of $S_{G}[X]$ and $G^{\prime} \in S_{G}[X]$ such that $G^{\prime} H \in R_{G}[X]$. Then, $G^{\prime} \in R_{G}[X]$.

Proof. We set anew $X^{(1,0)}=X, X^{(0,1)}=Y$. We may suppose $o_{X}\left(G^{\prime}\right)$ $=o_{Y}\left(G^{\prime}\right)=o_{X}(H)=o_{Y}(H)=0$, where " $o_{X}()$ " is the order as to $X$. Set $G^{\prime}=$ $g_{0}+g_{1} Y+\cdots+g_{n} Y^{n}, H=h_{0}+h_{1} Y+\cdots+h_{m} Y^{m}$ for $g_{i}, h_{j} \in S[X]$. Set $k=$ $\max \left\{\operatorname{deg}_{X} g_{i}, \operatorname{deg}_{X} h_{j} ; l \leq i \leq n, 1 \leq j \leq m\right\}$. Take $l>k . \quad G^{\prime}\left(X, X^{l}\right)$ and $H(X$, $\left.X^{l}\right)$ are elements of $S[X]$ and $H\left(X, X^{l}\right)$ is monic and $G^{\prime}\left(X, X^{\prime}\right) H\left(X, X^{\prime}\right) \in R[X]$. Hence, by [13] (10.4), it follows $G^{\prime}\left(X, X^{l}\right) \in R[X]$. The coefficients of $g_{i}$ are those of $G^{\prime}\left(X, X^{l}\right)$; hence belong to $R . g_{i} \in R[X]$. Hence, $G^{\prime} \in R[X, Y]$.

Proposition 24. Let $S$ be a ring, $R$ a subring such that $R$ is integrally closed in $S, G=Z \oplus \cdots \oplus Z$ with the lexicographic order. Let $H$ be a monic element of $S_{G}[X]$ and $G^{\prime} \in S_{G}[X]$ such that $G^{\prime} H \in R_{G}[X]$. Then, $G^{\prime} \in R_{G}[X]$.

Proof. The induction on $n$. Suppose the proposition is OK till $n-1$. Set $X^{\left(0, \ldots, 0, \frac{1}{i}, \ldots, 0\right)}=X_{i}$. We may suppose $o_{X_{i}}\left(G^{\prime}\right)=o_{X_{i}}(H)=0$. Take a sufficiently large $m$ and substitute $X_{1}^{m}$ to $X_{2}$. Then, the coefficients of $G^{\prime}\left(X_{1}, X_{2}, \ldots\right.$, $\left.X_{n}\right)$ are those of $G^{\prime}\left(X_{1}, X_{1}^{m}, X_{3}, \ldots, X_{n}\right)$ and the coefficients of $H\left(X_{1}, X_{2}, \ldots, X_{n}\right)$ are those of $H\left(X_{1}, X_{1}^{m}, X_{3}, \ldots, X_{n}\right)$ and $H\left(X_{1}, X_{1}^{m}, X_{3}, \ldots, X_{n}\right)$ is monic. By the 
hypothesis, it follows $G^{\prime}\left(X_{1}, X_{1}^{m}, X_{3}, \ldots, X_{n}\right) \in R\left[X_{1}, X_{3}, \ldots, X_{n}\right]$. Hence, $G^{\prime}\left(X_{1}\right.$, $\left.X_{2}, X_{3}, \ldots, X_{n}\right) \in R\left[X_{1}, X_{2}, X_{3}, \ldots, X_{n}\right]$.

Lemma 25. Let $S$ be a ring, $R$ a subring such that $R$ is integrally closed in $S, G$ a totally ordered abelian group $(\neq 0), h$ a monic element of $S_{G}[X]$ and $g \in S_{G}[X]$ such that $g h \in R_{G}[X]$. Assume $G$ be of the torsion-free rank 2. Then, $g \in R_{G}[X]$.

Proof. (i) Let $\{x, y\}$ be real numbers which is rationally independent. Assume $k_{1} x+l_{1} y<\cdots<k_{n} x+l_{n} y$ for $k_{i}, l_{j} \in Z$. Then, there exist natural numbers $p, q$ such that $k_{1} p+l_{1} q<\cdots<k_{n} p+l_{n} q$. (ii) Let $\{x, y\}$ be nationally independent real numbers, $G=Z x+Z y$ with the usual order of real numbers. Then, our Lemma is OK. Set $X^{x}=X, X^{y}=Y$. We may suppose $o_{X}(g)=o_{Y}(g)=o_{X}(h)=o_{Y}(h)=0$. We arrange the powers of $g, h$ as follows: $k_{1} x+l_{1} y<\cdots<k_{n} x+l_{n} y$. Take $p, q$ of (i). Substitute $X \rightarrow Z^{p}, Y \rightarrow Z^{q}$ in $g(X, Y), h(X, Y)(Z$ is an indeterminate). The coefficients of $g$ are those of $g\left(Z^{p}, Z^{q}\right)$. Similar as to $h . h\left(Z^{p}, Z^{q}\right)$ is a monic of $S[Z]$ and $g\left(Z^{p}, Z^{q}\right) h\left(Z^{p}, Z^{q}\right) \in R[Z]$. Hence, it follows $g\left(Z^{p}, Z^{q}\right) \in R[Z]$. Hence, $g(X, Y) \in R[X, Y]$. Hence, $g \in R_{G}[X]$. (iii) We may suppose $G$ is finitely generated. When $G$ has proper isolated subgroup, $G$ is order-isomorphic with $Z \oplus Z$ with the lexicographic order. Our assertion follows from Prop. 24. When $G$ does not have proper isolated subgroups, $G$ is order-isomorphic with one in (ii). Hence, our assertion follows.

TheOREM 26. Let $S$ be a ring, $R$ a subring such that $R$ is integrally closed in $S, G$ a totally ordered abelian group $(\neq 0), h$ a monic of $S_{G}[X]$ and $g \in S_{G}[X]$ such that $g h \in R_{G}[X]$. Then, $g \in R_{G}[X]$.

Proof. We may suppose $G$ is finitely generated. When $G$ does not have proper isolated subgroups, the assertion follows as Lem. 25. (ii). Generally, $G$ is order isomorphic onto $H_{1} \oplus H_{2} \oplus \cdots \oplus H_{k}$ with the lexicographic order, where $H_{i}$ is a subgroup of the reals with the usual order. $H_{i}=Z \pi_{i 1}+\cdots+Z \pi_{i m(i)}$ where $\left\{\pi_{i 1}, \ldots, \pi_{i m(i)}\right\}$ is independent reals $>0$. Set $X^{\pi_{i j}}=X_{i j}$. We may suppose $o_{X_{i j}}(g)$ $=o_{X_{i j}}(h)=0$. When all $m(i)=1$, our assertion is over in Prop. 24. When $m(l)>1$ for some $l$. The powers of $g, h$ are of the form $h_{1}+\cdots+h_{k}\left(h_{i} \in H_{i}\right)$. Consider the set of $l$-components $h_{l}$ of $h_{1}+\cdots+h_{k}$ of powers of $g, h$. We arrange them as follows: $\quad h^{1}<h^{2}<\cdots<h^{a} . \quad h^{1}=\sum b_{1 i} \pi_{l 1}, \ldots, h^{a}=\sum b_{a i} \pi_{l i}$ for $b_{j i} \in Z$. Take natural numbers $p_{1}, \ldots, p_{m(1)}$ such that $\sum b_{1 i} p_{i}<\cdots<\sum b_{a i} p_{i}$. Substitute $X_{i i} \rightarrow X_{i_{1}}$ in $g, h$. By the induction hypothesis on the torsion-free rank of $G$, it follows $g\left(X_{11}, \ldots, X_{1 m(1)} ; \ldots ; X_{l 1}, \ldots, X_{l 1} ; \ldots ; X_{k 1}, \ldots, X_{k m(k)}\right) \in R\left[X_{11}, \ldots, X_{1 m(1)} ; \ldots\right.$; $\left.X_{l 1}, \ldots, X_{l m(l)} ; \ldots ; X_{k 1}, \ldots, X_{k m(k)}\right]$. Hence, $g\left(X_{11}, \ldots, X_{1 m(1)} ; \ldots ; X_{l 1}, \ldots, X_{\operatorname{lm}(l)} ; \ldots ;\right.$ $\left.X_{k 1}, \ldots, X_{k m(k)}\right) \in R\left[X_{11}, \ldots, X_{1 m(1)} ; \ldots ; X_{l 1}, \ldots, X_{l m(l)} ; \ldots ; X_{k 1}, \ldots, X_{k m(k)}\right]$. Hence, $g \in R_{G}[X]$. 


\section{§2. The Kronecker functions rings}

Proposition 1. Let a be a nonzero ideal of a domain $D$. Then, $\left(a D_{G}[X]\right)_{v}$ $=a_{v} D_{G}[X]$.

We take " $v$ " in respective rings.

Proof. By $[26] \S 9$, Prop. 3, it follows $\left(a D_{G}[X]\right)_{v} \supset a_{v}$. Hence, $\left(a D_{G}[X]\right)_{v}$ $\supset \mathfrak{a}_{v} D_{G}[X]$. Conversely, let $\left\{\left(a_{i} / b_{i}\right) D ; I\right\}$ be the set of principal fractional ideals containing a. $a_{v}=\cap\left(a_{i} / b_{i}\right) D$. Then, $\left(\cap\left(a_{i} / b_{i}\right) D\right) D_{G}[X]=\cap\left(a_{i} / b_{i}\right) D_{G}[X]$.

Hence, $a_{v} D_{G}[X]$ is a divisorial ideal containing $a D_{G}[X]$; hence contains the minimal divisorial ideal $\left(a D_{G}[X]\right)_{v}$ containing $\mathfrak{a} D_{G}[X]$.

Proposition 2. If $D_{G}(X)_{\text {loc }}$ is a Dedekind domain, then $D_{b}$ is a Dedekind domain.

The notations are of [26].

Proof. $D_{G}(X)_{\text {loc }}$ is a prüfer domain. By the proof of [26] \$8, Th. 14, $(3) \rightarrow(1), D$ is a prüfer domain. Hence, $D$ is integrally closed. By [26] $\S 8$, Th. 14, it follows $D_{G}(X)_{\text {loc }}=D_{b}$. Hence, $D_{b}$ is a Dedekind domain.

Proposition 3. If $D_{b}$ is noetherian, then $D_{b}$ is a principal ideal domain.

Proof. [26] \&8, Th. 2.

Proposition 4. If $D_{b}$ is a Dedekind domain, $D_{G}(X)_{\text {loc }}$ is also a Dedekind domain.

Proof. By [26] $\$ 4$, Th. 10, $D$ is an almost Dedekind domain; hence, prüfer. By [26] $\$ 8$, Th. 14, it follows $D_{G}(X)_{\text {loc }}=D_{b}$. Hence, $D_{G}(X)_{\text {loc }}$ is a Dedekind domain.

Lemma 5. Let * be a e.a.b. *-operation on a domain $D$. Let $F, G^{\prime} \in$ $K_{G}[X]-\{0\}$. Then, $\left(A_{F G^{\prime}}\right)^{*}=\left(A_{F} A_{G^{\prime}}\right)^{*}$.

Proof. [26] § 8, Lem. 1.

Proposition 6. Let $D$ be a v-domain. Then the followings are equivalent.

(1) $D$ is a prüfer v-multiplication ring.

(2) $D^{v}$ is a quotient ring of $D_{G}[X]$.

Proof. (1) $\rightarrow(2)$. Set $N=\left\{g \in D_{G}[X] ; 1 / g \in D^{v}\right\}$. Let $f / h \in D^{v},\left(A_{f}\right)_{v} \subset$ $\left(A_{h}\right)_{v} . \quad \operatorname{div} A_{h}+\operatorname{div}\left(u_{0}, \ldots, u_{n}\right)=\operatorname{div} D$ for $u_{i} \in K$. Set $U=u_{0}+u_{1} X^{\alpha}+\cdots+u_{n} X^{n \alpha}$ for $0 \neq \alpha \in G$. By Lem. 5 , it follows $\left(A_{h U}\right)_{v}=\left(A_{h} A_{U}\right)_{v}=D$. Hence, $h U \in N$. $\left(A_{f U}\right)_{v}=\left(A_{f} A_{U}\right)_{v} \subset\left(A_{h}\right)_{v}\left(A_{U}\right)_{v}=D$. Hence, $f U \in D_{G}[X]$. Hence, $f / h=(f U) /(h U)$ $\in D_{G}[X]_{N}$. Hence, $D^{v} \subset D_{G}[X]_{N} . \quad(2) \rightarrow(1)$. Then, $D^{v}=D_{G}[X]_{N}$. Consider 
$\operatorname{div}\left(x_{0}, x_{1}, \ldots, x_{n}\right)$ for $x_{i} \in K-\{0\} . \quad x_{i}=a_{i} / b$ for $a_{i}, b \in D$. Take $0 \neq \alpha \in G$. Set $f=a_{0}+a_{1} X^{\alpha}+\cdots+a_{n} X^{n \alpha}$. $a_{0} / f \in D^{\nu}$. Hence, $a_{0} / f=h / g$ for $g \in N . \quad\left(a_{0}\right)=$ $\left(A_{a_{0} g_{v}}=\left(A_{f h}\right)_{v}=\left(A_{f} A_{h}\right)_{v} . \operatorname{div}\left(x_{0}, \ldots, x_{n}\right)+\operatorname{div}\left(b / a_{0}\right) A_{h}=\operatorname{div} D\right.$.

Proposition 7. If there exists a G-powered Kronecker functions ring $D$ on a domain $D$ such that $D$ is a Krull domain, then $D$ is a Krull domain.

Proof. $D_{*} \cap K=D$.

Let $\left\{V_{\lambda} ; A\right\}$ be a family of valuation overrings of $D$ such that $D=\bigcap V_{\lambda}$. Let $L$ be an algebraic field extension of $K$ and let $\left\{W_{\sigma} ; \Sigma\right\}$ be the family of all valuation rings $W$ on $L$ such that $W \cap K \in\left\{V_{2} ; \Lambda\right\}$. Set $J=\cap W_{\sigma}$. Let $D_{*}$ be the $G$-powered Kronecker functions ring with respect to $\left\{X_{i} ; I\right\}$ of the $w$-operation on $D$ induced by $\left\{V_{\lambda} ; \Lambda\right\}$. Let $J_{*}$ be the $G$-powered Kronecker functions ring with respect to $\left\{X_{i} ; I\right\}$ of the $w$-operation on $J$ induced by $\left\{W_{\sigma} ; \Sigma\right.$,

THEOREM 8. $J_{*}$ is the integral closure of $D_{*}$ in $L_{G}\left(X_{i} ; I\right)$.

Proof. By [26] \&8, Th. 5, it follows $J_{*}=\bigcap_{\sigma} W_{\sigma}^{*}$ and $D_{*}=\bigcap_{\lambda} V_{\lambda}^{*}$, where $W_{\sigma}^{*}$, $V_{\lambda}^{*}$ are the trivial extensions of $W_{\sigma}, V_{\lambda}$ to $L_{G}\left(X_{i}^{\sigma} ; I\right), K_{G}\left(X_{i} ; I\right)$ respectively. Let $v_{\lambda}$ be the valuation for $V_{\lambda}$ and $w_{\sigma}$ be the valuation for $W_{\sigma} . \bigcap_{\sigma} W_{\sigma}^{*}$ is integrally closed. Let $f / g \in \cap W_{a}^{*}$ for $f, g \in J_{G}\left[X_{i} ; I\right]$. Let $H$ be the subgroup of $G$ generated by the powers of $f, g . \quad \mathrm{H}=Z \alpha_{1} \oplus \cdots \oplus Z \alpha_{n}$. Set $X_{i}^{\alpha_{i}}=X_{i j}$. Let $w_{\alpha}^{\prime}$ be the trivial extension of $w_{\sigma}$ to $L\left(X_{i j} ; i, j\right)$. $\quad w_{\alpha}^{\prime}(f / g) \geq 0$; hence $f / g$ is integral over $\bigcap_{\lambda} V_{\lambda}\left(X_{i j} ; i, j\right)_{\text {loc }}$ ([13] p. 409,15). Since $V_{\lambda}\left(X_{i j} ; i, j\right)_{\mathrm{loc}} \subset V_{\lambda}^{*}, f / g$ is integral over $\cap V_{\lambda}^{*}$. Hence, $J_{*}$ is the integral closure of $D_{*}$ in $L_{G}\left(X_{i} ; I\right)$.

Next, we state the direct proof of Th. 8 .

Let $f / g \in \cap W_{o}^{*} ; f, g \in J_{G}\left[X_{i} ; I\right]$. It suffices to show that $f / g$ is integral over $\cap V_{\sigma}^{*}$. We may suppose $\Lambda=1$ point: $\left\{V_{\lambda} ; \Lambda\right\}=\{V\}$. (i) When $L / K$ is purely inseparable. Let $w^{*}$ be any extension of $v^{*}$ to $L_{G}\left(X_{i} ; I\right)$. $(f / g)^{p e} \in K_{G}\left(X_{i} ; I\right)$ for some e. $\quad p^{e} w^{*}(f / g)=w^{*}\left((f / g)^{p^{e}}\right)=v^{*}\left((f / g)^{p^{e}}\right)=w_{\sigma}^{*}\left((f / g)^{p^{e}}\right)=p^{e} w_{\sigma}^{*}(f / g) \geq 0$ for any $\sigma$. Hence, $f / g$ is integral over $V^{*}$. (ii) When $L / K$ is finite normal separable. Let $w^{*}$ be any extension of $v^{*}$ to $L_{G}\left(X_{i} ; I\right)$. Let $[L: K]=n$ and $\left\{\tau_{1}, \ldots, \tau_{n}\right\}$ be the Galois group of $L / K$. By [26] $\S 9$, Th. $2, L_{G}\left(X_{i} ; I\right) / K_{G}\left(X_{i} ; I\right)$ is a Galois extension of degree $n$ and its Galois group is $\left\{\tau_{1}^{*}, \ldots, \tau_{n}^{*}\right\}$ where $\tau_{j}^{*}$ is the natural extension of $\tau_{j}$ to $L_{G}\left(X_{i} ; I\right)$. Fix $\sigma \in \Sigma$. $w^{*}=w_{\sigma}^{*} \tau_{j}^{*}$ for some $j$. Hence, $w^{*}(f / g)$ $\geq 0$; hence $f / g$ is integral over $V^{*}$. (iii) When $L / K$ is finite separable. Let $M$ be the minimal normal extension of $K$ containing $L$. Let $u$ be any extension of $v$ to $M . \quad u \mid L=w_{\text {o }}$ for some $\sigma . \quad u^{*}(f / g)=w_{\sigma}^{*}(f / g) \geq 0$. By (ii), $f / g$ is integral over $V^{*}$. (iv) When $L / K$ is finite. Let $M$ be the separable closure of $K$ in $L$. Let $\left\{U_{\alpha} ; \mathbf{a}\right\},\left\{u_{a} ; \mathfrak{a}\right\}$ be the extensions of $V, v$ to $M$ respectively. By (i), $f / g$ is integral over $U_{a}^{*}$. Hence, $f / g$ is integral over $\cap U_{\alpha}^{*}$. By (iii), $\cap U_{\alpha}^{*}$ is integral over $V^{*}$. 
Hence, $f / g$ is integral over $l^{*} *$. (v) When $L / K$ is algebraic. Let $M$ be the extension $K$ (coefficients of $f, g$ ). Let $\left\{U_{\alpha} ; a\right\},\left\{u_{\alpha} ; a\right\}$ be the extensions of $V, v$ to $M$ respectively. $u_{\alpha}=w_{a} \mid M$ for some $\sigma . \quad u_{\alpha}^{*}(f / g)=w_{\alpha}^{*}(f / g) \geq 0$. Hence, by (iv), $f / g$ is integral over $V^{*}$.

\section{\$3. The noetherian}

Proposition 1. Let $(A, \mathrm{in})$ be a local ring such that $A_{G}(X)_{\text {loc }}$ is regular local. Then, $A$ is regular local.

The terminology "local ring" is a ring with only one maximal ideal.

Proof. Let $\mathfrak{a}, \mathfrak{b}$ be ideals of $A$ such that $\mathfrak{a} \subsetneq \mathfrak{b}$. If $\mathfrak{a} A_{G}(X)_{\text {loc }}=\mathfrak{b} A_{G}(X)_{\text {loc }}$, it follows $\mathfrak{a}=\mathfrak{b}$ by [26] $\S 7$, Prop. 2. Hence, $A$ is noetherian. By [26] $\S 7$, Prop. 2, it follows $d=\operatorname{dim} A \leq \operatorname{dim} A_{G}(X)_{\text {loc }} \quad\left(a_{1}, \ldots, a_{d}\right) A$ is im-primary for $a_{i} \in A$. By [26] \$7. Prop. 2, $\left(a_{1}, \ldots, a_{d}\right) A_{G}(X)_{\text {loc }}$ is $\mathfrak{m} A_{G}(X)_{\text {loc }}$-primary. Hence, $d \geq$ $\operatorname{dim} A_{G}(X)_{\text {loc }}$. Hence, $\operatorname{dim} A=\operatorname{dim} A_{G}(X)_{\text {loc }}$ We confer [45] chap. 8, \$11, Th. 25. The vector space (m $\left.A_{G}(X)_{\text {loc }}\right) /\left(\mathrm{m}^{2} A_{G}(X)_{\text {loc }}\right)$ over $A_{G}(X)_{\text {loc }} /\left(\mathrm{m} A_{G}(X)_{\text {loc }}\right)$ is of dimension $d$ and is generated by $\left\{x+m^{2} A_{G}(X)_{\text {loc }} ; x \in m\right\}$. Hence, there exists a basis $\left\{x_{j}+\mathfrak{m}^{2} A_{G}(X)_{\mathrm{loc}} ; x_{j} \in \mathfrak{m}, 1 \leq j \leq d\right\}$. Hence, $\mathfrak{m} A_{G}(X)_{\mathrm{loc}}=\left(x_{1}, \ldots, x_{d}\right) A_{G}$ $(X)_{\text {loc. }}$ Let $x \in \mathfrak{m}$. $x=\sum\left(x_{i} f_{i}\right) / g$ for $f_{i} \in A_{G}[X]$ and $A_{G}[X] \ni g \in \mathfrak{m} A_{G}[X]$. It follows $x \in\left(x_{1}, \ldots, x_{d}\right) A$. Hence, $m=\left(x_{1}, \ldots, x_{d}\right) A$. Hence, $A$ is regular.

Corollary 2. Assume $A_{G}[X]_{\mathfrak{F}}$ is regular for any prime $\mathfrak{P}$ of $A_{G}[X]$. Then, $A_{\mathfrak{p}}$ is regular for any prime $\mathfrak{p}$ of $A$.

Proof. Let $\mathfrak{p}$ be a prime ideal of $A . \quad \mathfrak{p} A_{G}[X]$ is a prime ideal of $A_{G}[X]$. Hence, $A_{G}[X]_{p A_{G}[X]}$ is regular. $A_{G}[X]_{p A_{G}[X]}=\left(A_{p}\right)_{G}[X]_{\mathrm{p}(A p) G[X]}$. By Prop. 1, $A$ is regular.

Proposition 3. If $A_{G}[X]_{\mathfrak{R}}$ is regular for every prime ideal $\mathfrak{P}$ of $A_{\mathfrak{G}}[X]$, then the torsion-free rank of $G$ is finite.

Proof. Prop. 16.

THEOREM 4. $A_{G}[X]_{\mathfrak{B}}$ is regular for every prime ideal $\mathfrak{P}$ of $A_{G}[X]$ if and only if the torsion-free rank of $G$ is finite, $A_{\mu}$ is regular for every prime ideal $\mathfrak{p}$ of $A$, $(G / F)_{p}$ is finite for each $p \in \Omega$.

The notations are of [23].

Proof. Prop. 3 and [23] Th., (4).

If all of overrings of $A$ are noetherian, $A$ is said a $C$-ring [10].

Proposition 5. If $A_{G}[X]$ is a C-ring, then $A$ is also a $C$-ring. 
Proof. Let $B$ be an overring of $A . \quad B_{G}[X]$ is an overring of $A_{G}[X]$. Hence, $B_{G}[X]$ is noetherian. Hence, $B$ is noetherian [9]. Hence, $A$ is a $C$-ring.

Proposition 6. If $A_{G}[X]$ is a $C$-ring, then $\operatorname{dim} A=0$ and $G \cong Z$.

Proof. $A$ is noetherian. $G=Z \alpha_{1} \oplus \cdots \oplus Z \alpha_{n} . \quad$ Set $X^{\alpha_{i}}=X_{i}, A_{Z}\left[X_{1}, \ldots\right.$, $\left.X_{n-1}\right]=B . \quad B_{Z}\left[X_{n}\right]$ is a $C$-ring. Suppose $\operatorname{dim} B>0$. There exist prime ideals $\mathfrak{p} \nsupseteq \mathfrak{q}$ of $B . \quad\left(\mathfrak{p}, 1-X_{n}\right) \supsetneq \mathfrak{p} B_{Z}\left[X_{n}\right] \supsetneq \mathfrak{q} B\left[X_{n}\right]$. We confer [10]. $B_{Z}\left[X_{n}\right]$ is of the restricted dimension $\geq 2$; a contradiction. Hence, the dimension of $B$ is 0 . Hence, it follows $n=1$. Hence, $G \cong Z$ and $\operatorname{dim} A=0$.

TheOREM 7. $A_{G}[X]$ is a $C$-ring if and only if $G \cong Z$ and $A$ is a direct sum of a finite number of fields.

Proof. (1) If $A_{Z}[X]$ is a $C$-ring and $A$ is a primary ring, then $A$ is a field. By Prop. 6, it follows $\operatorname{dim} A=0$. By Prop. 5, $A$ is a $C$-ring. The nilradical $\mathfrak{p}$ of $A$ is a prime ideal. $(\mathfrak{p}, 1-X)$ is a maximal ideal of $A_{Z}[X] . A_{Z}[X]_{(p, 1-X)}$ is not a $Z$-ring; hence without nilpotents. Let $a \in \mathfrak{p} . \quad a g=0$ for $A_{Z}[X] \ni g \bar{\epsilon}$ $(\mathfrak{p}, 1-X) . \quad a s=0$ for $A \ni s \bar{\in} \mathfrak{p}$. Hence, it follows $a=0$. Hence, $\mathfrak{p}=0$. Hence, $A$ is a field. (2) $A_{z}[X]$ is a $C$-ring if and only if $A$ is a direct sum of a finite number of fields. $A$ is of dimension 0 and a $C$-ring. $(0)=\mathfrak{q}_{1} \cap \cdots \cap \mathfrak{q}_{n}, \sqrt{\mathfrak{q}_{i}}=\mathfrak{p}_{i}, \mathfrak{p}_{i} \neq \mathfrak{p}_{j}$ $(i \neq j)$ for primary ideals $\mathfrak{q}_{i}$ of $A . \quad A \cong A / \mathfrak{q}_{1} \oplus \cdots \oplus A / \mathfrak{q}_{n} . \quad A_{Z}[X] \cong\left(A / \mathfrak{q}_{1}\right)_{2}[X]$ $\oplus \cdots \oplus\left(A / \mathfrak{q}_{n}\right)_{z}[X] . \quad\left(A / \mathfrak{q}_{i}\right)_{z}[X]$ is a $C$-ring. By (1), $A / \mathfrak{q}_{i}$ is a field. Conversely, let $A=k_{1} \oplus \cdots \oplus k_{n}$ for fields $k_{i}$. By [10] Th. $1, A_{z}[X]$ is a $C$-ring. (3) The necessity follows from Prop. 6.

Proposition 8. If $A_{G}[X]$ is a Macaulay ring of [29], then so is $A$.

Proof. We may suppose $G=Z$. By [26] $\$$, Prop. 3, $A$ is a locally Macaulay ring of [29]. Suppose $h t \mathrm{~m}<\operatorname{dim} A$ for some maximal ideal $m$ of $A$. There exist maximal chains containing $(1-X)$ of prime ideals of $A_{z}[X]$ such that $\mathfrak{P}_{0} \supsetneq \mathfrak{P}_{1} \supsetneq \ldots \supsetneq \mathfrak{P}_{n}, \mathfrak{Q}_{0} \supsetneq \mathbb{Q}_{1} \supsetneq \ldots \supsetneq \mathfrak{Q}_{m}, n \neq m$. There arise maximal chains in $A_{z}[X]$ of lengths $n+1$ and $m+1$. This is a contradiction.

REMARK 9. Even if $A$ is a Macaulay ring of [29], $A_{Z}[X]$ need not be so.

$A$ Counter Example. Let $L, M$ be fields, $A=L \oplus M$. We confer [29] (25.12).

Lemma 10. If $A$ is noetherian, then $\operatorname{dim} A_{z}\left[X_{1}, \ldots, X_{n}\right]=n+\operatorname{dim} A$.

Proof. $\operatorname{dim} A[X]=1+\operatorname{dim} A$.

Proposition 11. Let A be a Macaulay ring of [29]. Suppose (*) "for every maximal ideal $\mathfrak{M}$ of $A_{z}\left[X_{1}, \ldots, X_{n}\right], \mathfrak{M} \cap A$ is a maximal ideal of $A$ ". Then, $A_{Z}\left[X_{1}, \ldots, X_{n}\right]$ is a Macaulay ring of $[29]$. 
Proof. Let $\mathfrak{M}$ be a maximal ideal of $A_{Z}\left[X_{1}, \ldots, X_{n}\right]$ and let $\mathfrak{M} \cap A=\mathfrak{n}$. By Lem. 10, it follows ht $\mathfrak{M} \geq n+$ ht $\mathrm{n}=n+\operatorname{dim} A=\operatorname{dim} A_{z}\left[X_{1}, \ldots, X_{n}\right] \geq$ ht $\mathfrak{M}$. Hence, ht $\mathfrak{M l}=\operatorname{dim} A_{Z}\left[X_{1}, \ldots, X_{n}\right]$.

Proposition 12. If $A, A_{Z}\left[X_{1}, \ldots, X_{n}\right]$ are Macaulay rings of [29] then (*) holds.

Proof. Suppose (*) does not hold. There exists a maximal ideal $\mathfrak{M}$ of $A_{\mathbf{Z}}\left[X_{1}, \ldots, X_{n}\right]$ such that $\mathfrak{M} \cap A=n$ is not a maximal ideal of $A$. $n+\mathrm{ht} \mathfrak{n}=$ $\operatorname{dim}\left(A_{n}\right)_{2}\left[X_{1}, \ldots, X_{n}\right] \geq$ ht $\mathfrak{M}_{\mathrm{n}} \geq n+h t n A_{\mathrm{n}}=n+$ ht $\mathrm{n} . \quad$ ht $\mathfrak{M}=$ ht $\mathfrak{M}_{\mathrm{n}}=n+$ ht $\mathrm{n}<n+$ $\operatorname{dim} A=\operatorname{dim} A_{Z}\left[X_{1}, \ldots, X_{n}\right]$. Hence, $A_{Z}\left[X_{1}, \ldots, X_{n}\right]$ is not a Macaulay ring of [29].

Proposition 13. $A_{Z}\left[X_{1}, \ldots, X_{n}\right]$ is a Macaulay ring of [29] if and only if $A$ is a Macaulay ring of [29] and (*) holds.

Proposition 14. There exists a maximal ideal $\mathfrak{M l}$ of $D_{Z}\left[X_{1}, \ldots, X_{n}\right]$ such that $\mathfrak{M} \cap D=0$ if and only if $D\left[a^{-1}\right]$ is a field for some $0 \neq a \in D$.

The proof is similar with that of [29] (14.10).

Theorem 15. $A_{G}[X]$ is a Macaulay ring of [29] if and only if $A$ is a Macaulay ring of [29], $G$ is finitely generated, $A$ has no prime ideals $p$ such that (1) $\mathfrak{p}$ is not a maximal ideal, (2) $(A / \mathfrak{p})\left[\bar{a}^{-1}\right]$ is a field for some a $\bar{E} \mathfrak{p}$.

Proof. The necessity. By Prop. 13, $A$ is a Macaulay ring of [29] and (*) holds. Suppose there exist $\mathfrak{p}$ satisfying (1), (2). Apply Prop. 14 to $A / \mathfrak{p}$. There exists a maximal ideal $\mathfrak{M}$ of $A_{\mathfrak{Z}}\left[X_{1}, \ldots, X_{n}\right]$ such that $\mathfrak{M} \cap A=\mathfrak{p}$, a contradiction. The sufficiency. Suppose $(*)$ does not hold. There exists a maximal ideal $\mathfrak{M}^{\prime}$ of $A_{Z}\left[X_{1}, \ldots, X_{n}\right]$ such that $\mathfrak{M}^{\prime} \cap A=\mathfrak{p}$ is not a maximal ideal of $A$. Prop. 14 leads to a contradiction.

If $A_{\mathfrak{p}}$ is noetherian for every prime ideal $\mathfrak{p}$ of $A, A$ is said to be locally noetherian.

Proposition 16. If $A_{G}[X]$ is locally noetherian, the torsion-free rank of $G$ is finite.

Proof. Suppose the torsion-free rank is infinite. Let $\left\{\alpha_{\lambda} ; \Lambda\right\}$ be a maximal independent subset of $G$. Set $H=\sum_{\lambda} Z \alpha_{\lambda}$. Take $\left\{\alpha_{1}, \alpha_{2}, \alpha_{3}, \ldots\right\} \subset\left\{\alpha_{\lambda} ; \Lambda\right\}$. $\left(1-X^{a_{1}}, \ldots, 1-X^{a_{n}}\right) A_{H}[X]=\mathfrak{p}_{n}$ is a prime ideal of $A_{H}[X]$. Take a prime ideal $\mathfrak{P}_{1}$ of $A_{G}[X]$ over $\mathfrak{p}_{1}$. Take a prime ideal $\mathfrak{P}_{2} \supset \mathfrak{P}_{1}$ of $A_{G}[X]$ over $\mathfrak{p}_{2}$. Take $\mathfrak{P}_{1}, \mathfrak{P}_{2}, \mathfrak{P}_{3}, \ldots$ like this. $\bigcup_{1}^{\infty} \mathfrak{P}_{i}=\mathfrak{P}$ is a prime ideal of $A_{G}[X] . A_{G}[X]_{\mathfrak{P}}$ is not noetherian, a contradiction.

THEOREM 17. $A_{G}[X]$ is locally noetherian if and only if $G$ is of finite torsion- 
free rank, $A$ is locally noetherian, $(G / F)_{p}$ is finite for each $p \in \Omega$.

The notations are of [23].

Proof. Prop. 16 and [23] Th., (1).

The next is on the global Gorenstein rings [21] p. 164.

Proposition 18. If $A_{G}[X]$ is a global Gorenstein ring, then $A$ is a global Gorenstein ring.

Proof. $A$ is noetherian. Let $\mathrm{m}$ be a maximal ideal of $A . \quad(\mathrm{m}, 1-X)$ is a maximal ideal of $A_{Z}[X]$. Hence, $A_{Z}[X]_{(\mathrm{m}, 1-X)}$ is a Gorenstein ring. $(1-X) / 1$ is a non-zerodivisor of $A_{Z}[X]_{(\mathrm{m}, 1-X)}$. Hence, $A_{Z}[X]_{(\mathrm{m}, 1-X)} /(1-X) A_{Z}[X]_{(\mathrm{m}, 1-X)}$ is Gorenstein (cf. [21] p. 164, 13). This last ring is isomorphic with $A_{\mathrm{m}}$. Hence, $A$ is a global Gorenstein ring. Next, $G \cong Z \oplus \cdots \oplus Z . \quad A_{G}[X] \cong A_{Z}\left[X_{1}, \ldots, X_{n}\right]$. From the above, $A_{Z}\left[X_{1}, \ldots, X_{n-1}\right]$ is a global Gorenstcin ring. Like this, $A$ is a global Gorenstein ring.

Proposition 19. $A_{G}[X]$ is a global Gorenstein ring if and only if $A$ is a global Gorenstein ring and $G$ is finitely generated.

Proof. [23] Th., (3).

The next is on the $\mathrm{J}-0$ [27] (32.B).

Proposition 20. If $A$ is $J-0$, then $A_{Z}[X]$ is $J-0$.

Proof. There exists $a \in A$ such that $\phi \neq D(a) \subset \operatorname{Spec}(A)$ and $A_{\mathfrak{p}}$ is regular for every $\mathfrak{p} \in D(a)$. Set $D^{\prime}(a)=\left\{\mathfrak{P} \in \operatorname{Spec}\left(A_{Z}[X]\right) ; \mathfrak{P} \bar{\jmath} a\right\} . \quad D^{\prime}(a) \neq \phi . \quad$ Let $\mathfrak{P} \in$ $D^{\prime}(a), \mathfrak{P} \cap A=\mathfrak{p}$. Since $\mathfrak{p} \bar{\ni} a, A_{\mathfrak{p}}$ is regular. $A_{Z}[X]_{\mathfrak{F}} \cong\left(A_{\mathfrak{p}}\right)_{Z}[X]_{\mathfrak{\beta}}$, for a prime ideal $\mathfrak{P}^{\prime}$ of $\left(A_{\mathfrak{p}}\right)_{Z}[X]$. Since $\left(A_{\mathfrak{p}}\right)[X]$ is regular, $\left(A_{\mathfrak{p}}\right)_{Z}[X]_{\mathfrak{p}}$ is regular. Hence, $A_{Z}[X]_{\mathfrak{P}}$ is regular. Hence, $A_{Z}[X]$ is $J-0$.

The next is on the $\mathrm{J}-2$ [27] (32.B).

Proposition 21. If $A_{G}[X]$ is $J-2, A$ is also $J-2$.

Proof. Let $\mathfrak{p}$ be a prime ideal of $A$. Then, there exists a prime ideal $\mathfrak{P}$ of $A_{G}[X]$ such that $A_{G}[X] / \mathfrak{P}=A / \mathfrak{p}$.

Lemma 22. If $A$ is $J-2, A_{Z}[X]$ is also $J-2$.

Proof. A finitely generated $A_{Z}[X]$-algebra is a finitely generated $A$-algebra.

Proposition 23. $A_{G}[X]$ is $J-2$ if and only if $A$ is $J-2$ and $G$ is finitely generated.

Proof. Prop. 21, Lem. 22 and the results of [9]. 


\section{§4. The pseudo-prüfer}

Lemma 1. Let $\pi \in G$ be of ht $(0,0,0, \ldots), K$ a field, $a, b \in K-\{0\}$. Then, $a+b X^{\pi}$ is irreducible in $K_{G}[X]$.

Proof. Similar with that of [24] Lem. 2.2.

Lemma 2. Let $\pi \in G$ be of ht $(0,0,0, \ldots), f \in D_{Z_{\pi}}[X]-D$. If $f$ is irreducible in $D_{Z_{\pi}}[X]$, then $f$ is irreducible in $D_{G}[X]$.

Proof. The proof appears in the proof of Prop. 5.

Proposition 3. Let $D$ be a prüfer domain, $G$ contain $a$ ht $(0,0,0, \ldots)$ element $\pi$. If every irreducible element of $D_{G}[X]$ is also irreducible in $K_{G}[X]$, then $D$ is a bezout domain.

Proof. Suppose $D$ is not bezout. There exist $a, b \in D$ such that $(a, b)$ is not principal. $(a, b)(c, d)=D$ for $c, d \in K$. Let $f=\left(a+b X^{\pi}\right)\left(c+d X^{\pi}\right)$. By [26] $\S 2$, Th. 11, $A_{f}=(a, b)(c, d)=D$. Hence, $f \in D_{G}[X] . f$ is not irreducible in $K_{G}[X]$. Hence, $f$ is not irreducible in $D_{G}[X]$. By Lem. $2, f$ is not irreducible in $D_{Z \pi}[X]$. Let $f=f_{1} \cdots f_{n}$ be the irreducible decomposition of $f$ in $D_{Z \pi}[X] ; n \geq 2$. $f_{i}$ is irreducible in $K_{G}[X]$; hence irreducible in $K_{Z_{\pi}}[X] . f=f_{1} \cdots f_{n}$ is the irreducible decomposition in $K_{Z \pi}[X]$. By Lem. $1, a+b x^{\pi}$ is associated with, say $f_{1}$ in $K_{Z \pi}[X]$.

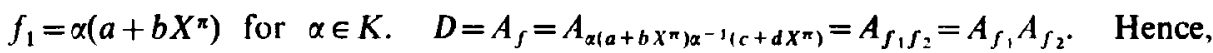
$A_{f_{1}}=D . \quad(\alpha a, \alpha b)=D . \quad(a, b)=\left(\alpha^{-1}\right) ;$ a contradiction.

Proposition 4. Let $D$ be an integrally closed domain, $\pi a$ ht $(0,0,0, \ldots)$ element of $G$. Assume that every prime ideal $\mathfrak{P}$ of $D_{G}[X]$ such that $\mathfrak{P} \cap D=0$ is principal. Then $D$ is a pseudo-bezout domain.

Proof. Let $a, b \in D-\{0\}$. Take a maximal $¥$ among ideals of $D_{G}[X]$ containing $a+b X^{\pi}$ and not intersecting with $D-\{0\} . \quad \mathfrak{P}$ is prime. $\mathfrak{B}=(f)$ for $f \in D_{G}[X] . \quad a+b X^{\pi}=f g$ for $g \in D_{G}[X]$. By Lem. $1, g$ is a unit of $K_{G}[X] . g=$ $d \in D . \quad D_{G}[X] \ni f=a / d+(b / d) X^{\pi}=a^{\prime}+b^{\prime} X^{\pi}$. Since $(f)$ is prime, $\operatorname{GCD}\left(a^{\prime}, b^{\prime}\right)$ is 1. By [26] $\S 6$, Prop. 1 (3), it follows $\operatorname{div}(a, b)=\operatorname{div} A_{a+b X^{\pi}}=\operatorname{div} A_{d\left(a^{\prime}+b^{\prime} X^{\pi}\right)}$ $\operatorname{div}(d)+\operatorname{div}\left(a^{\prime}, b^{\prime}\right)=\operatorname{div}(d)$. Hence, $d=\operatorname{GCD}(a, b)$.

Propositron 5. Let $D$ be a prüfer v-multiplication ring, $\pi a$ ht $(0,0,0, \ldots)$ element of $G$. Assume that every irreducible $f \in D_{G}[X]-D$ in $D_{G}[X]$ such that $\left(A_{f}\right)_{v}=D$ is irreducible in $K_{G}[X]$. Then $D$ is pseudo-bezout.

$K$ is the quotient field of $D$.

Proof. Let $a, b \in D-\{0\} . \operatorname{div}(a, b)+\operatorname{div}\left(x_{0}, \ldots, x_{n}\right)=\operatorname{div} D$ for $x_{i} \in K$. 
Set $f=\left(a+b X^{\pi}\right)\left(x_{0}+x_{1} X^{\pi}+\cdots+x_{n} X^{n \pi}\right)$. By [26] $\$ 6$, Prop. 1, it follows $\left(A_{f}\right)_{v}$ $=\left((a, b)\left(x_{0}, \ldots, x_{n}\right)\right)_{v}=D$. Hence, $f \in D_{G}[X]$ and $f$ is reducible in $D_{G}[X]$. $f$ has not nonunit constant factors in $D_{G}[X]$. Let $f=g h$ be a proper decomposition in $D_{G}[X]$ and let $H$ be the subgroup generated by $\pi$ and the powers of $g, h$. By [24] (2.1), $Z \pi$ is a direct summand of $H . \quad H=Z \pi \oplus Z \alpha_{1} \oplus \cdots \oplus Z \alpha_{m}$. We may suppose $g, h \in D_{z_{\pi}}[X]$. Let $g=g_{1} \cdots g_{k}, h=h_{1} \cdots h_{l}$ be irreducible decompositions in $D_{Z_{\pi}}[X] ; k, l \geq 1 . \quad g_{i}, h_{j}$ are irreducible in $D_{G}[X]$. Since $\left(A_{g_{i}}\right)_{v}=\left(A_{h_{j}}\right)_{v}=D, g_{i}$ and $h_{j}$ are irreducible in $K_{G}[X]$. Since $f=g_{1} \cdots g_{k} h_{1} \cdots h_{l}$ is the irreducible decomposition in $K_{Z_{\pi}}[X], a+b X^{\pi}$ is associated with, say $g_{i}$ in $K_{Z_{\pi}}[X] . \quad\left(a+b x^{\pi}\right)(t / s)$ $=g_{i}$ for $s, t \in D$. Set $(t / s) a=c \in D,(t / s) b=d \in D . \quad \operatorname{div}(a, b)=\operatorname{div}(s / t)+\operatorname{div}(c, d)$ $=\operatorname{div}(s / t)$. Hence, $s / t=\operatorname{GCD}(a, b)$.

The next is on the $(n)^{\prime}([13]$ p. 305).

Proposition 6. If $A_{G}[X]$ is $(n)^{\prime}, A$ is $(n)^{\prime}$.

The next is on the $(n)([13]$ p. 305).

Proposition 7. If $A_{G}[X]$ is $(n)$, then $A$ is $(n)$.

Remark 8. If $A_{G}[X]$ is (2), then $A$ is von Neumann regular.

Proof. If $A$ has nonzero nilpotents, there exists $b \neq 0$ such that $b^{2}=0$. Let $0 \neq \alpha \in G . \quad b\left(1+X^{\alpha}\right)=\left(1+X^{\alpha}\right)^{2} g$ for some $g . \quad b=\left(1+X^{\alpha}\right) g$; this is obviously impossible. Suppose $A$ have nonunit non-zerodivisor a. $a\left(1+X^{x}\right)=a^{2} f+$ $\left(1+X^{\alpha}\right)^{2} g$ for some $f, g . \quad 1+X^{\alpha}=a f+\left(1+X^{\alpha}\right)^{2} g^{\prime}$ for some $g^{\prime}$. This is also impossible.

Proposition 9. $D_{z}[X]$ is (2) if and only if $D$ is a field.

Proposition 10. $D_{G}[X]$ is (2) if and only if $D$ is a field and $G \subset Q$ in the sense of isomorphism.

$Q$ is the rational numbers.

Proof. The necessity. By Remark $8, D=K$ is a field. Let $f, g \in K_{G}[X]$ $-\{0\}$. There exists the GCD $h$ of $f, g$ in $K_{G}[X]$ ([24] Prop. 1.4). $f=h f_{1}$, $g=h g_{1}, f_{1} g_{1}=f_{1}^{2} f^{\prime}+g_{1}^{2} g^{\prime}$. Let $H$ be the subgroup generated by the powers of $f_{1}, g_{1}, f^{\prime}, g^{\prime} . K_{H}[X]$ is factorial. $1=f_{1} f^{\prime \prime}+g_{1} g^{\prime \prime}$ for some $f^{\prime \prime}, g^{\prime \prime}$. Then, $(f, g)=(h)$. Similarly, every finitely generated ideal of $K_{G}[X]$ is principal; especially $K_{G}[X]$ is prüfer. Hence, $G$ is isomorphic into $Q$ ([16]). The sufficiency. Let $f, g \in K_{G}[X]-\{0\}$. There exists the GCD $h$ of $f, g . f=h f_{1}, g=h g_{1}$. There exists $n>0$ such that $f_{1}, g_{1} \in K_{Z}[Y]$, where $Y=X^{1 / n} . \quad 1=f_{1} f^{\prime}+g_{1} g^{\prime}$. It follows $f g=f^{2}\left(g_{1} f^{\prime}\right)+g^{2}\left(f_{1} g^{\prime}\right)$. Hence, $K_{G}[X]$ is (2).

Proposition 11. If $A_{G}[X]$ is $(n)$, then $A$ is von Neumann regular. 
Proof. Suppose $A$ has nonzero nilpotent. Then there exists $0 \neq b \in A$ such that $b^{2}=0$. Take $0 \neq \alpha \in G . \quad b\left(1+X^{\alpha}\right)^{n-1}=\left(1+X^{\alpha}\right)^{n} g$ for some $g . \quad b=$ $\left(1+X^{\alpha}\right) g$; this is impossible. Hence, $A$ has zero nilradical. Next, suppose $A$ has nonunit non-zerodivisor a. $a\left(1+X^{a}\right)^{n-1}=a^{n} f^{\prime}+\left(1+X^{a}\right) g^{\prime}$ for some $f^{\prime}, g^{\prime}$. $\left(1+X^{\alpha}\right)^{n-1}=a^{n-1} f^{\prime}+\left(1+X^{\alpha}\right)^{n} g^{\prime \prime}$ for some $g^{\prime \prime}$. This is impossible.

Proposition 12. Let $A$ be a noetherian ring, $n>1$. Then, $A_{G}[X]$ is $(n)$ if and only if $A$ is von Neumann regular and $G$ is isomorphic into $Q$.

Proof. The necessity. By Prop. $11, A$ is von Neumann regular. $(A / \mathfrak{p})_{G}[X]$ is $(n)$ for a prime ideal $\mathfrak{p}$ of $A$; hence $G$ is isomorphic into $Q$ (Th. 14). The sufficiency. $A \cong k_{1} \oplus \cdots \oplus k_{n}$ for fields $k_{i}$. By Th. $14,\left(k_{i}\right)_{G}[X]$ is $(n)$. Hence, $A_{G}[X]$ is $(n)$.

Proposition 13. If $A_{G}[X]$ is $(n)$, then $G$ is isomorphic into $Q$.

Proof. Th. 14.

Theorem 14. Let $n>1 . D_{G}[X]$ is $(n)$ if and only if $D$ is a field and $G$ is isomorphic into $Q$.

Proof. The case $n=2$ is over in Prop. 10. We may suppose $n>2$. The necessity. By Prop. $11, D=K$ is a field. Let $f, g \in K_{G}[X]-\{0\}$. There exists $\operatorname{GCD}(f, g)=h([24](1.4))$. $f=h f_{1}, g=h g_{1}, f_{1} g_{1}^{n-1}=f_{1}^{n} f^{\prime}+g_{1}^{n} g^{\prime}$. Let $H$ be the subgroup generated by the powers of $f_{1}, g_{1}, f^{\prime}, g^{\prime} . K_{H}[X]$ is factorial. $g_{1}^{n-1}$ $=f_{1}^{n-1} f^{\prime}+g_{1}^{n} g^{\prime \prime}, 1=f_{1}^{n-1} f^{\prime \prime}+g_{1} g^{\prime \prime}$. Hence, $(f, g)=(h)$. Hence, every finitely generated ideal of $K_{G}[X]$ is principal. By [16], $G$ is isomorphic into $Q$. The sufficiency. $D=K$. Let $f, g \in K_{G}[X]-\{0\}$. There exists $\operatorname{GCD}(f, g)=h . f=h f_{1}$, $g=h g_{1}$. There exists $n$ such that $f_{1}, g_{1} \in K_{2}[Y]$, where $Y=X^{1 / n} . \quad 1=\operatorname{GCD}\left(f_{1}^{n}\right.$, $\left.g_{1}^{n}\right) . \quad 1=f_{1}^{n} f^{\prime}+g_{1}^{n} g^{\prime}$. For $0 \leq k \leq n$, it follows $f^{k} g^{n-k}=f^{n} f^{\prime \prime}+g^{n} g^{\prime \prime}$. Hence, $(f, g)^{n}=\left(f^{n}, g^{n}\right)$.

The next is on the $n$-generator property ([13] p. 320$)$.

Proposition 15. If $A_{G}[X]$ has n-generator property, then $A$ has n-generator property.

THEOREM 16. Let $n>0 . D_{G}[X]$ has n-generator property if and only if $D$ is $a$ field and $G$ is isomorphic into $Q$.

Proof. The necessity. Let $\bar{D}$ be the integral closure of $D . \bar{D}_{G}[X]$ is the integral closure of $D_{G}[X]$ [24] (4.4). By [14] Cor. $3, \bar{D}$ is a field and $G$ is isomorphic into $Q$. Hence $D$ is a field.

Proposition 17. Let $n>0$. If $A_{G}[X]$ has n-generator property, then $\operatorname{dim} A$ $=0$ and $G$ is isomorphic into $Q$. 
Proof. Th. 16.

The next is on the GCD-ring [13] p. 76.

Proposition 18. If $A_{G}[X]$ is a GCD-ring, then $A$ is also a GCD-ring.

Proof. Similar with that of Prop. 20.

Remark 19. Even if $A$ is a GCD-ring, $A_{Z}[X]$ need not be a GCD-ring.

A Counter Example. $A=Z /(4) . \quad f=(1+X)(3+X), g=(1+X)^{2} \in A_{Z}[X]$. Suppose there exist GCD $h$ of $f, g . h \in(1+X) \cap(3+X)$. As seen in Remark 21, $h$ is of the form either $(1+X)(3+X) f^{\prime}$ or $(1+X)\left\{(3+X) f^{\prime}+2\right\}$. Suppose $h=$ $(1+X)(3+X) f^{\prime} . \quad(1+X)^{2}=(1+X)(3+X) f^{\prime} f_{1} . \quad 1+X=(3+X) f^{\prime} f_{1} ; \quad$ a contradiction. The rest is similar.

The next is on the LCM-ring [13] p. 76.

Proposition 20. If $A_{G}[X]$ is a LCM-ring, then $A$ is a LCM-ring.

Proof. Let $\varphi$ be the canonical homomorphism of $A_{G}[X]$ to $A$. Let $a, b \in A$ and $f$ the LCM of $a, b$ in $A_{G}[X]$. Then, $\varphi(f)$ is the LCM of $a, b$ in $A$.

ReMARK 21. Even if $A$ is a LCM-ring, $A_{Z}[X]$ need not be a LCM-ring.

A Counter Example. $\quad A=Z /(4) . \quad f \in(1+X) \cap(3+X)$ is associated with either $(1+X)(3+X) g$ or $(3+X)\{(1+X) g+2\}$. Suppose there exist $h$ such that $(1+X) \cap(3+X)=(h)$. Then, we see a contradiction.

\section{§5. The extension $K_{G}[X] / k_{c}[X]$}

Let $K / k$ be a field extension. We concern in this section with the properties in the extension $K_{G}[X] / k_{G}[X]$.

Proposition 1. Let $K / k$ be fields, a an ideal of $k_{G}[X]$. Then, $a K_{G}[X]$ $\cap k_{G}[X]=\mathfrak{a}$.

Proof. Let $\left\{u_{\lambda} ; \Lambda\right\}$ be a linear basis of $K / k$; let $u_{\lambda_{0}}=1 . \quad\left\{u_{\lambda} ; \Lambda\right\}$ is linearly independent over $k_{G}[X]$. Every element of $K_{G}[X]$ is uniquely expressed as $\sum f_{\lambda} u_{\lambda}$, $f_{\lambda} \in k_{G}[X]$, where almost all $f_{\lambda}$ are zero. $\mathbf{a} K_{G}[X]=\sum \mathbf{a} u_{\lambda}$. Let $f \in \mathfrak{a} K_{G}[X] \cap$ $k_{G}[X] . \quad f=\sum f_{\lambda} u_{\lambda}$ for $f_{\lambda} \in \mathfrak{a} . \quad\left(f_{\lambda_{0}}-f\right)+\sum_{\lambda \neq \lambda_{0}} f_{\lambda} u_{\lambda}=0$. Hence, $f=f_{\lambda_{0}} \in \mathfrak{a}$.

Proposition 2. Let $K / k$ be fields, $\mathfrak{a}, \mathfrak{b}$ ideals of $k_{G}[X]$. Then, $(\mathfrak{a} \cap \mathfrak{b}) K_{G}[X]$ $=a K_{G}[X] \cap b K_{G}[X]$.

Proof. Let $F \in \mathfrak{a} K_{G}[X] \cap \mathfrak{b} K_{G}[X]$. Under the notations of the proof of Prop. 1, we set $F=\sum f_{\lambda} u_{\lambda}, f_{\lambda} \in \mathfrak{a}, F=\sum g_{\lambda} u_{\lambda}, g_{\lambda} \in \mathbf{b}$. Since $\left\{u_{\lambda} ; \Lambda\right\}$ is lintearly independent over $k_{G}[X]$, it follows $f_{\lambda}=g_{\lambda} \in \mathfrak{a} \cap \mathfrak{b}$. Hence, $F \in(\mathfrak{a} \cap \mathfrak{b}) K_{G}[X]$. 
Proposition 3. Let $\mathfrak{a}, \mathfrak{b}$ ideals of $k_{G}[X]$. Then,

$$
(\mathfrak{a}: \mathfrak{b})_{k_{G}[X]} K_{G}[X]=\left(\mathfrak{a} K_{G}[X]: \mathfrak{b} K_{G}[X]\right)_{K_{G}[X]} \text {. }
$$

Proof. Let $F \in\left(\mathfrak{a} K_{G}[X]: \mathfrak{b} K_{G}[X]\right)_{K_{G}[X]}$. With the notations of Prop. 1, $F=\sum f_{\lambda} u_{\lambda}, f_{\lambda} \in k_{G}[X]$. Let $g \in \mathfrak{b} . \quad F g=\sum g_{\lambda} u_{\lambda}$ for $g_{\lambda} \in \mathfrak{a}$. Since $\sum f_{\lambda} g u_{\lambda}=$ $\sum g_{\lambda} u_{\lambda}$, it follows $f_{\lambda} g=g_{\lambda}$. Hence, $f_{\lambda} \in(\mathfrak{a}: \mathfrak{b})_{k_{G}[X]} . \quad F=\sum f_{\lambda} u_{\lambda} \in(\mathfrak{a}: \mathfrak{b})_{k_{G}[X]} K_{G}[X]$.

Proposition 4. Assume $\left\{t_{\lambda} ; 1\right\}$ be algebraically independent subset of $K$ over $k$ and $K=k\left(t_{\lambda} ; \Lambda\right), \mathfrak{p}$ a prime ideal of $k_{G}[X]$. Then, (1) $p K_{G}[X]$ is a prime ideal of $K_{G}[X]$. (2) $\left\{t_{\lambda} ; \Lambda\right\}$ is algebraically independent over the quotient field of $k_{G}[X] / p$. (3) The quotient field of $K_{G}[X] / p K_{G}[X]$ is the field obtained by adjoining $\left\{t_{\lambda} ; \Lambda\right\}$ to the quotient field of $k_{G}[X] / p$.

Proof. We set $k_{G}[X]=R, K_{G}[X]=S, p S=p^{e}$. (1) Let $F^{\prime} G^{\prime} \in \mathfrak{p}^{e}$ for $F^{\prime}$, $G^{\prime} \in S$. There exists a finite subset $\left\{t_{1}, \ldots, t_{n}\right\} \subset\left\{t_{\lambda} ; A\right\}$ such that $F^{\prime}, G^{\prime} \in k\left(t_{1}, \ldots\right.$, $\left.t_{n}\right)_{G}[X]$. By Prop. 1, we may suppose $\Lambda$ is one point; $\left\{t_{\lambda} ; \Lambda\right\}=\{t\}$. We may suppose $F^{\prime}, G^{\prime} \in k[t]_{G}[X] . \quad F^{\prime}=\sum f_{j} t^{j}, G^{\prime}=\sum g_{j} t^{j}$ for $f_{j}, g_{j} \in R$. Suppose $F^{\prime}$ $\bar{\epsilon} \mathfrak{p}^{e}, G^{\prime} \bar{\epsilon} \mathfrak{p}^{e}$. Set $\min \left\{j ; f_{j} \bar{\epsilon} \mathfrak{p}\right\}=\alpha, \min \left\{j ; g_{j} \bar{E} \mathfrak{p}\right\}=\beta$. Take a linear base $\left\{u_{\lambda} ; A\right\}$ containing $\left\{1, t, t^{2}, \ldots\right\}$ of $K / k . \quad F^{\prime} G^{\prime}$ is uniquely written as the form $\sum h_{\lambda} u_{\lambda}, h_{\lambda} \in \mathfrak{p}$. Then, $f_{\alpha} g_{\beta} \in \mathfrak{p}$. Hence, $f_{\alpha} \in \mathfrak{p}$ or $g_{\beta} \in \mathfrak{p} ;$ a contradiction. (2) Let $F(\mathfrak{p}), F\left(p^{e}\right)$ the quotient fields of $R / p, S / p^{e}$ respectively. Suppose $\left\{t_{\lambda} ; \Lambda\right\}$ is algebraically dependent over $F(p)$. There is a finite subset $\left\{t_{1}, \ldots, t_{n}\right\} \subset\left\{t_{\lambda} ; \Lambda\right\}$ such that $\left\{t_{1}, \ldots, t_{n}\right\}$ is algebraically dependent over $F(\mathfrak{p})$. Set $K^{\prime}=k\left(t_{1}, \ldots, t_{n}\right)$, $S^{\prime}=K_{G}^{\prime}[X]$. Then, $p S^{\prime}$ is a prime ideal of $S^{\prime}$. Hence, we may suppose $\Lambda$ is one point; $\left\{t_{\lambda} ; \Lambda\right\}=\{t\}$. Let $f_{m} t^{m}+\cdots+\bar{f}_{0}=0\left(f_{j} \in R, \bar{f}_{m} \neq 0\right)$ be the minimal equation of $t$ over $F(\mathfrak{p}) . \quad f_{m} t^{m}+f_{m-1} t^{m-1}+\cdots+f_{0} \in \mathfrak{p}^{e}$. If we set like $\left\{u_{\lambda} ; \Lambda\right\} \supset\left\{1, t, t^{2}, \ldots\right\}$ in the proof of Prop. 1, it follows $f_{j} \in \mathfrak{p}$. A contradiction. (3) is immediate.

Proposition 5. In Prop. 4, we have transc. $\operatorname{deg}_{k} F(\mathfrak{p})=\operatorname{transc} . \operatorname{deg}_{K} F\left(p^{e}\right)$.

The notations are of in the proof of Prop. 4.

Proof. By Prop. $4,\{K, F(p)\}$ is free over $k$. There is a transcendence basis of $F(\mathfrak{p}) / k$ of the form $\left\{X^{\beta}+\mathfrak{p} ; \beta \in \mathfrak{B}\right\}$. Then $\left\{X^{\beta}+\mathfrak{p}^{\mathfrak{e}} ; \beta \in \mathfrak{B}\right\}$ is transcendental over $K$. Then, $\left\{X^{\beta}+\mathfrak{p}^{e} ; \beta \in \mathfrak{B}\right\}$ is a transcendence basis of $F\left(\mathfrak{p}^{e}\right) / K$.

Proposition 6. Assume $K / k$ be purely-transcendental extension fields. Let $\mathfrak{q}$ be a p-primary ideal of $k_{G}[X]$. Then, $\mathfrak{q} K_{G}[X]$ is a $\mathfrak{p} K_{G}[X]$-primary ideal of $K_{G}[X]$.

Proof. By Prop. 4, it follows $\sqrt{\mathfrak{q} K_{G}[X]}=\mathfrak{p} K_{G}[X]$. We may suppose $K=k(t)$. Let $F^{\prime} G^{\prime} \in q^{e}$ for $F^{\prime}, G^{\prime} \in S$ and $G^{\prime} \bar{\in} q^{e}$. We may suppose $F^{\prime}, G^{\prime}$ $\in k[t]_{G}[X]$. By [29] (6.15), $\mathfrak{q} R[t]$ is primary in $R[t]$. Hence, $F^{\prime n} \in \mathfrak{q}^{e}$ for some $n$. 
Proposition 7. Let $K$ be an extension field of a field $k, \mathfrak{p}$ a prime ideal of $k_{G}[X]$. Then, (1) $\mathfrak{p} K_{G}[X]$ is primary in $K_{G}[X]$ if and only if every zerodivisor of $\left(k_{G}[X] / p\right) \otimes_{k} K$ is nilpotent; (2) $p K_{G}[X]$ is prime in $K_{G}[X]$ if and only if $\left(k_{G}[X] / \mathfrak{p}\right) \otimes_{k} K$ is a domain; (3) $\sqrt{\mathfrak{p} K_{G}[X]}=\mathfrak{p} K_{G}[X]$ if and only if the nilradical of $\left(k_{G}[X] / \mathfrak{p}\right) \otimes_{k} K$ is zero.

Proof. (1) Adjoin $\otimes_{k} K$ to the exact sequence $0 \rightarrow \mathfrak{p} \rightarrow k_{G}[X] \rightarrow k_{G}[X] / \mathfrak{p} \rightarrow 0$. (2) $K_{G}[X] / \mathfrak{p} K_{G}[X] \cong\left(k_{G}[X] / \mathfrak{p}\right) \otimes_{k} K$.

Proposition 8. Let $K / k$ be fields, $\mathfrak{p}$ a prime ideal of $k_{G}[X]$. (1) If $K / k$ is a separable extension ([44] p. 113, Def. 2), then $\sqrt{\mathfrak{p} K_{G}[X]}=\mathfrak{p} K_{G}[X]$. (2) If the quotient field of $k_{G}[X] / p$ is separable over $k$, then $\sqrt{p K_{G}[X]}=p K_{G}[X]$. (3) If $k$ is perfect, then $\sqrt{\mathfrak{p} K_{G}[X]}=\mathfrak{p} K_{G}[X]$.

Proof. (1) By [44] p. 195, Th. 39, the nilradical of $F(p) \otimes_{k} K$ is zero. Since $(R / \mathfrak{p}) \otimes K \subset F(\mathfrak{p}) \otimes K$, the nilradical of $(R / \mathfrak{p}) \otimes K$ is zero. Hence, the nilradical of $S / p^{e}$ is zero.

Proposition 9. Let $k$ be a field, $\mathfrak{p}$ a prime ideal of $k_{G}[X]$. Assume $\sqrt{\mathfrak{p} K_{G}[X]}=\mathfrak{p} K_{G}[X]$ for every extension field $K$ of $k$. Then, $F(\mathfrak{p})$ is a separable extension of $k$.

Proof. We may suppose the characteristic of $k$ be $p>0$. Set $K=k^{1 / p}$. $p^{e}=p K_{G}[X]$ is a primary ideal of $K_{G}[X]$ (Prop. 13). Hence, $p^{e}$ is a prime ideal of $K_{G}[X] . \quad K$ and $k_{G}[X] / \mathfrak{p}$ are linearly disjoint over $k$ in $K_{G}[X] / p^{e} . \quad F\left(p^{e}\right)$ is a common extension of $K, F(\mathfrak{p}) ; K$ and $F(\mathfrak{p})$ are linearly disjoint over $k$ in $F\left(\mathfrak{p}^{e}\right)$. Hence, $F(\mathfrak{p})$ is separable over $k$.

Lemмa 10. Let $\mathfrak{q}$ be a primary ideal of $k_{G}[X]$ and $\sqrt{\mathfrak{q}}=\mathfrak{p}$. Assume $G$ be finitely generated and $\mathfrak{P}$ a prime ideal of $q K_{G}[X]=q^{e}$ in $K_{G}[X]$. Then, $\mathfrak{P} \cap k_{\boldsymbol{G}}[X]=\mathfrak{p}$.

Proof. $\quad q^{e}: \mathfrak{P} \geq q^{e}$. Hence, $q^{e}: \mathfrak{P}^{c e} \supsetneq q^{e} \quad$ where $\mathfrak{P}^{c}=\mathfrak{P} \cap k_{G}[X] . \quad$ By Prop. 3, $\mathfrak{q}^{e}: \mathfrak{P}^{c e}=(\mathfrak{q}: \mathfrak{P} c)^{e}$. Hence, $\left(\mathfrak{q}: \mathfrak{P}^{c}\right)^{e} \mathfrak{q}^{e}$. By Prop. 1, $\mathfrak{q}: \mathfrak{P} \mathfrak{q} \mathfrak{q}$. Hence, $\mathfrak{P} c=\mathfrak{p}$.

Proposition 11. Let $K$ be a purely-inseparable field extension of $k, q$ a primary ideal of $k_{G}[X], G$ finitely generated. Then, $\mathfrak{q} K_{G}[X]$ is a primary ideal of $K_{G}[X]$.

Proof. $K_{G}[X]$ is noetherian. Let $\mathfrak{P}, \mathfrak{P}^{\prime}$ be prime ideals of $\mathrm{q} K_{G}[X]$.

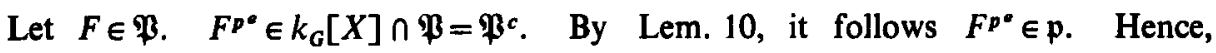

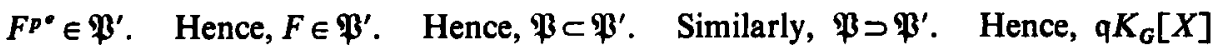
has only one prime ideal. 
LemMa 12. Assume $H$ be a subgroup of $G, q$ a primary ideal of $k_{G}[X]$. Then, $q \cap k_{H}[X]$ is a primary ideal of $k_{H}[X]$.

Proposition 13. Let $K / k$ be a purely-inseparable field extension, q $a$ primary ideal of $k_{G}[X]$. Then, $\mathfrak{q} K_{G}[X]=q^{e}$ is a primary ideal of $K_{G}[X]$.

Proof. Let $F^{\prime} G^{\prime} \in q^{e}$ for $F^{\prime}, G^{\prime} \in K_{G}[X], G^{\prime} \in q^{e} . \quad F^{\prime} G^{\prime}=\sum f_{i} F_{i}$ for $f_{i} \in \mathfrak{q}$, $F_{i} \in K_{G}[X]$. Let $H$ be the subgroup generated by the powers of $F^{\prime}, G^{\prime}, f_{1}, \ldots, f_{m}$, $F_{1}, \ldots, F_{m}$. By Lem. 12, $q \cap k_{H}[X]$ is a primary ideal of $k_{H}[X]$. By Prop. 11, $\left(q \cap k_{H}[X]\right) K_{H}[X]$ is a primary ideal of $K_{H}[X]$. Since $F^{\prime}, G^{\prime} \in K_{H}[X], F^{\prime} G^{\prime}$ $\epsilon\left(q \cap k_{H}[X]\right) K_{H}[X], G^{\prime} \bar{\epsilon}\left(q \cap k_{H}[X]\right) K_{H}[X]$, it follows $F^{\prime n} \in\left(q \cap k_{H}[X]\right) K_{H}[X]$ for some $n$. $F^{\prime n} \in q^{e}$. Hence, $q^{e}$ is primary.

Proposition 14. Let $\mathfrak{p}$ be a prime ideal of $k_{G}[X]$. Assume $k$ is quasimaximally algebraic in $F(p)$. Then, $\mathfrak{p} K_{G}[X]$ is primary for every extension $K / k$.

The terminology "quasi-maximally algebraic" means that every separably algebraic element of $F(p)$ over $k$ is contained in $k$ ([45] p. 227).

Proof. Reduce to the case where $G$ is finitely generated. $k_{G}[X]=k_{z}\left[X_{1}, \ldots\right.$, $\left.X_{n}\right]$. $k$ is q. m.a. in the quotient field of $k\left[X_{1}, \ldots, X_{n}\right] /\left(p \cap k\left[X_{1}, \ldots, X_{n}\right]\right)$. By [45] V 11, Th. 38, $\left(p \cap k\left[X_{1}, \ldots, X_{n}\right]\right) K\left[X_{1}, \ldots, X_{n}\right]$ is a primary ideal of $K\left[X_{1}, \ldots\right.$, $\left.X_{n}\right]$. Hence, $p K_{Z}\left[X_{1}, \ldots, X_{n}\right]$ is a primary ideal of $K_{Z}\left[X_{1}, \ldots, X_{n}\right]$.

Proposition 15. Let $\mathfrak{p}$ be a prime ideal of $k_{G}[X]$. Assume $\mathfrak{p}^{e}=\mathfrak{p} K_{G}[X]$ is primary in $K_{G}[X]$ for every extension $K / k$. Then $k$ is quasi-maximally algebraic in $F(\mathfrak{p})$.

Proof. Let $\alpha \in F(\mathfrak{p})$ be separably algebraic over $k$. Let $G(T)=T^{q}+a_{q-1} T^{q-1}$ $+\cdots+a_{0}$ be the minimal polynomial of $\alpha$ over $k$. Let $c_{1}^{\prime}, \ldots, c_{q}^{\prime}$ be the roots of $G(T)$. Set $K=k\left(c_{1}^{\prime}, \ldots, c_{q}^{\prime}\right) . \quad \alpha=\bar{A} / \bar{B}$ where $\bar{A}, \bar{B} \in k_{G}[X] / \mathfrak{p}$ for $A, B \in k_{G}[X] . A^{q}+$ $a_{q-1} A^{q-1} B+\cdots+a_{0} B^{q} \in \mathfrak{p} \subset \mathfrak{p}^{e} . \quad \sqrt{\mathfrak{p}^{e}}=\mathfrak{P}$ is a prime ideal of $K_{G}[X] . \quad\left(A-c_{1}^{\prime} B\right)$ $\cdots\left(A-c_{q}^{\prime} B\right) \in \mathfrak{p}^{e} \subset \mathfrak{P}$. Hence, $A-c_{i_{0}}^{\prime} B \in \mathfrak{P}$ for some $i_{0}$. For every automorphism $\sigma$ of $K / k$, we have $\mathfrak{P}^{\sigma}=\mathfrak{P}$. Hence, $A-c_{i}^{\prime} B \in \mathfrak{P}$ for every $i$. If $q \geq 2$, it follows $\left(c_{2}^{\prime}-c_{1}^{\prime}\right) B \in \mathfrak{P}$. Since $\mathfrak{P} \overline{c_{2}^{\prime}} \boldsymbol{c}_{2}^{\prime}-c_{1}^{\prime}$ (Prop. 1), it follows $B \in \mathfrak{P}$. $B^{m} \in \mathfrak{P}^{\mathrm{e}}$ for some $m . \quad B^{m} \in \mathfrak{p}^{e} \cap k_{G}[X]=\mathfrak{p}$ (Prop. 1). Hence, $B \in \mathfrak{p} ;$ a contradiction. Hence, $q=1$. Hence, $\alpha \in k$.

Proposition 16. Let $\mathfrak{p}$ be a prime ideal of $k_{G}[X]$. Assume $F(\mathfrak{p})$ be regular over $k$. Then $\mathfrak{p} K_{G}[X]$ is prime in $K_{G}[X]$ for every extension $K / k$.

Proof. Reduce to the case where $G$ is finitely generated. $k_{G}[X]=k_{z}\left[X_{1}, \ldots\right.$, $\left.X_{n}\right]$. Next, reduce to the fact that $\left(\mathfrak{p} \cap k\left[X_{1}, \ldots, X_{n}\right]\right) K\left[X_{1}, \ldots, X_{n}\right]$ is prime in $K\left[X_{1}, \ldots, X_{n}\right]$ (cf. [45] V 11, Th. 39). 
Proposition 17. Let $\mathfrak{p}$ be a prime ideal of $k_{\mathrm{G}}[X]$. Assume $\mathfrak{y}^{\mathfrak{e}}=\mathfrak{p} K_{\mathrm{G}}[X]$ be prime in $K_{G}[X]$ for every extension $K / k$. Then, $F(\mathfrak{p})$ is a regular extension of $k$.

Proof. By Prop. 9, $F(\mathfrak{p})$ is a separable extension over $k$. By Prop. 15, $k$ is quasi-maximally algebraic in $F(\mathfrak{p})$.

\section{§6. The Hilbert}

Proposition 1. If $A_{G}[X]$ is Hilbert, then $A$ is also Hilbert.

Proof. $A$ is a homomorphic image of $A_{G}[X]$.

Proposition 2. Assume $\mathfrak{M} \cap A$ is a maximal ideal of $A$ for every maximal ideal $\mathfrak{M}$ of $A_{G}[X]$. Then, $A$ is a Hilbert ring.

Proof. Let $\mathfrak{p}$ be a prime ideal of $A$ and $\mathfrak{p} \bar{\ni} a \in A$. Take $0 \neq \alpha \in G$. There exists a maximal ideal $\mathfrak{M}$ of $A_{\mathfrak{G}}[X]$ containing $\left(\mathfrak{p}, 1+a X^{\alpha}\right) A_{\mathfrak{G}}[X]$. $\mathfrak{M} \ni a$. The maximal ideal $\mathfrak{M} \cap A$ of $A$ does not contain $a$ and contains $\mathfrak{p}$. Hence, $\mathfrak{p}$ is an inter section of maximal ideals.

Proposition 3. Let $A$ be a Hilbert ring. Assume $M \cap A$ is maximal in $A$ for every free $G$ and for every maximal ideal $M$ of $A_{G}[X]$. Then $M \cap A$ is maximal in $A$ for any $G$ and for any maximal ideal $M$ of $A_{G}[X]$.

Proof: Consider any $G$. Let $\left\{\alpha_{\lambda} ; \Lambda\right\}$ be a maximal independent subset of $G$. Set $H=\sum_{\lambda} Z \alpha_{\lambda}$. Then $A_{\mathrm{G}}[X]$ is integral over $A_{I I}[X]$.

Proposition 4. Let $A$ be a Hilbert ring. Let $M$ be a maximal ideal of $A_{\mathrm{Z}}[X]$. Then, $A \cap M$ is a maximal ideal of $A$.

Proof. Let $D$ be a Hilbert domain. Let $M$ be a maximal ideal of $D_{z}[X]$ such that $D \cap M=0$. Set $x=\bar{X} \in D_{z}[X] / M, y=\bar{X}^{-1} \in D_{z}[X] / M . \quad D[x, y]$ is a field which is algebraic over $D$. If $D$ has zero pseudoradical, then $D[x, y]$ has also zero pseudoradical. This is impossible. Hence, $D$ has nonzero pseudoradical; that is to say, $D$ is a $G$-domain. Since $D$ is Hilbert, $D$ is a field.

Remark 5. Assume $D$ be a Hilbert domain, $G$ be free, $M$ a maximal ideal of $D_{G}[X]$. Then, $D \cap M$ need not be maximal in $D$.

A Counter Example. $X_{1}, X_{2}, \ldots ; T$ indeterminates. Let $\left\{\varphi_{1}, \varphi_{2}, \varphi_{3}, \ldots\right\}$ be the set of units of $Z[T]_{(T)}$. Define a homomorphism $Z\left[X_{1}, X_{2}, \ldots\right] \rightarrow Z[T]_{(T)}$ by $X_{i} \mapsto \varphi_{i}$. Extend the homomorphism to $\theta: Z_{Z}\left[X_{1}, X_{2}, \ldots\right] \rightarrow Z[T]_{(T)}$. Similar with Example $7 ; \theta$ is a surjection. Set $M=\theta^{-1}\left(T Z[T]_{(T)}\right) . \quad M \cap Z=0$. We set $D=Z, G=\underbrace{Z \oplus Z \oplus \cdots}_{N_{0}}$. 
Example 6. Let $k$ be a field. Then $k_{Q}[X]$ is Hilbert.

Proof Set $Y=X^{1} . \quad k_{Z}[Y]$ is 1-dimensional. $k_{Q}[X]$ is integral over $k_{Z}[Y]$. Hence, $\operatorname{dim} k_{Q}[X]=1$. The Jacobson radical of $k_{Q}[X]$ is zero $([26] \S 3$, Th. 3).

EXample 7. Assume $A$ be a countable ring, $G=\underbrace{(\oplus)}_{N_{0}-Z}$. Then, $A_{G}[X]$ is not Hilbert.

Proof. At first, let $k$ be a countable field, $X_{1}, X_{2}, \ldots ; T$ a set of indeterminates. Let $\left\{\varphi_{1}, \varphi_{2}, \varphi_{3}, \ldots\right\}$ be the set of units of $k[T]_{(T)}$. We define a homomorphism $\theta: k_{Z}\left[X_{1}, X_{2}, \ldots\right] \rightarrow k[T]_{(T)}$ by $X_{i} \mapsto \varphi_{i} . \quad 1-T=\varphi_{i}$ for some $i . \quad \theta\left(1-X_{i}\right)$ $=T$. Hence, $\theta$ is a surjection. Set $\operatorname{ker}(\theta)=\mathfrak{P} . \quad k_{z}\left[X_{1}, X_{2}, \ldots\right] / \mathfrak{B} \cong k[T]_{(T)}$. $J$-rad. $\left(k_{Z}\left[X_{1}, X_{2}, \ldots\right] / \mathfrak{P}\right) \neq 0 . \quad k_{Z}\left[X_{1}, X_{2}, \ldots\right]$ is not Hilbert. Suppose $A_{G}[X]$ be Hilbert. Let $p$ be a maximal ideal of $A$. Then, $(A / \mathfrak{p})_{Z}\left[X_{1}, X_{2}, \ldots\right] \cong A_{G}[X] /$ $\mathfrak{p} A_{G}[X]$ is also a Hilbert ring; a contradiction.

ThEOREM 8. Assume $G$ be finitely generated, $a$ an ideal of $A$. Then, a is a $G$-ideal of $A$ if and only if $\mathfrak{M} \cap A=a$ for some maximal ideal $\mathfrak{M}$ of $A_{\mathrm{G}}[X]$.

Proof. (1) For every $G$-ideal $\mathfrak{P}$ of $A_{Z}[X], A \cap \mathfrak{B}$ is a $G$-ideal of $A$. Set $A[X] \cap \mathfrak{P}=\mathfrak{P}_{0}$. Denote the total quotient ring by $q(\quad) . \quad q\left(A_{Z}[X] / \mathfrak{P}\right)=\left(A_{Z}[X] /\right.$ $\mathfrak{P})\left[\xi_{1}, \ldots, \xi_{m}\right]$ for $\xi_{i} \in q\left(A_{\mathbf{Z}}[X] / \mathfrak{P}\right) . \quad A_{z}[X] / \mathfrak{P}=\left(A[X] / \mathfrak{P}_{0}\right)\left[\overline{X^{-1}}\right]$, where $\overline{X^{-1}}$ $=X^{-1}+\mathfrak{P}$. Hence, $\mathfrak{P}_{0}$ is a $G$-ideal of $A[X]$. By $[13]$ p. $389,15, A \cap \mathfrak{P}=A \cap \mathfrak{P}_{0}$ is a $G$-ideal of $A$. (2) Let $D$ be a $G$-domain. Then, there exists a maximal ideal $\mathfrak{M}$ of $D_{z}[X]$ such that $\mathfrak{M} \cap D=0 . q(D)=K=D\left[u^{-1}\right]$ for $u \in D$. There exists a homomorphism $\theta: D_{z}[X] \rightarrow K, X \mapsto u^{-1}$. Set $\mathfrak{M}=\operatorname{ker}(\theta)$. (3) Let $\mathfrak{p}$ be a $G$-ideal of $A$. Then, there exists a maximal ideal $\mathfrak{M}$ of $A_{Z}[X]$ such that $\mathfrak{M} \cap A=p$. $A_{G}[X] \cong A_{Z}\left[X_{1}, \ldots, X_{n}\right]$. The assertion follows from (3), (1).

Proposition 9. Let $R$ be a ring. Assume $M \cap R$ be a $G$-ideal of $R$ for every free $G$ and for every maximal $M$ of $R_{G}[X]$. Then, $M \cap R$ is a $G$-ideal of $R$ for any $G$ and for every maximal $M$ of $R_{G}[X]$.

Proof. Consider any $G$. Let $\left\{\alpha_{\lambda} ; A\right\}$ be a maximal independent subset of G. Set $H=\sum_{\lambda} Z \alpha_{\lambda}$. Let $M$ be a maximal ideal of $R_{G}[X] . \quad R_{H}[X] \cap M$ is a maximal ideal of $\hat{R}_{H}[X]$. Hence, $\left(R_{H}[X] \cap M\right) \cap R$ is a $G$-ideal of $R$.

Remark 10. Assume $G$ be free and $M$ a maximal ideal of $A_{G}[X]$. Then, $M \cap A$ need not be a $G$-ideal of $A$.

\section{A Counter Example. Remark 5.}

Proposition 11. Let $A$ be a ring. Assume every $G$-ideal of $A$ is a contraction of a maximal ideal of $A_{G}[X]$ for every free $G$. Then, every $G$-ideal of $A$ is a contraction of a maximal ideal of $A_{G}[X]$ for any $G$. 
Proor. Let $\left\{\alpha_{\lambda} ; \Lambda\right\}$ be a maximal independent subset of $G$ and let $H=$ $\sum_{\lambda} Z \alpha_{\lambda}$. Let $I$ be a $G$-ideal of $A$. There exists a maximal ideal $M^{\prime}$ of $A_{H}[X]$ such that $M^{\prime} \cap A=I$. Since $A_{G}[X]$ is integral over $A_{H}[X]$, the assertion is $\mathrm{OK}$.

Proposition 12. Assume $G$ be free. Then every G-ideal $\mathfrak{p}$ of $A$ is a contraction of a maximal ideal of $A_{G}[X]$.

Proof. $G=\oplus Z \alpha_{\lambda}$. Fix $\lambda_{0} \in \Lambda$. Set $X^{\alpha_{\lambda}}=X_{\lambda}$. By Th. 8, there exists a maximal ideal $M_{0}^{\lambda}$ of $A_{Z}\left[X_{\lambda_{0}}\right]$ such that $M_{0} \cap A=$ p. Set $B=A_{Z}\left[X_{\lambda_{0}}\right] . A_{G}[X]$ $=B_{Z}\left[X_{\dot{\lambda}} ; \lambda \in \Lambda-\left\{\lambda_{0}\right\}\right]$. Take a maximal ideal $M \supset M_{0}$ of $A_{G}[X]$. Then, $M \cap A$ $=\mathfrak{p}$.

Proposition 13. Assume $G$ be finitely generated. Let $M$ be a maximal ideal of $K_{G}[X]$. Then, $K_{G}[X] / M$ is algebraic over $K$.

Remark 14. Assume $G$ be free, $M$ a maximal ideal of $k_{G}[X]$. Then, $k_{G}[X] /$ $M$ need not be algebraic over $k$.

A Counter Example. $Q(T)-\{0\}=\left\{\varphi_{1}, \varphi_{2}, \varphi_{3}, \ldots\right\}$. Define a homomorphism $\theta: Q_{z}\left[X_{1}, X_{2}, \ldots\right] \rightarrow Q(T)$ by $X_{i} \mapsto \varphi_{i}$. Set $M=\operatorname{ker}(\theta)$. We set $G=$

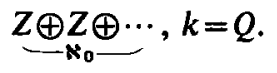

Proposition 15. Let $A$ be a ring. Assume $\mathfrak{P} \cap A$ be a $G$-ideal of $A$ for every free $G$ and for every $G$-ideal $\mathfrak{P}$ of $A_{G}[X]$. Then, $\mathfrak{P} \cap A$ is a $G$-ideal of $A$ for any $G$ and for every $G$-ideal $\mathfrak{P}$ of $A_{G}[X]$.

Proof. (1) Let $E$ be a $G$-domain, $D$ a subdomain such that $E$ is integral over $D$ Then, $D$ is also a $G$-domain. There exists $0 \neq b \in E$ such that $b$ is contained in every nonzero prime ideal $\mathfrak{P}$ of $E . \quad b^{n}+b^{n-1} a_{1}+\cdots+a_{n}=0$ for $a_{i} \in D$. We may suppose $a_{n} \neq 0$. Let $\mathfrak{p}$ be a nonzero prime ideal of $D$. There exists a prime ideal $\mathfrak{P}$ of $E$ over $\mathfrak{p}$. Then, $a_{n} \in \mathfrak{P} \cap D=\mathfrak{p}$. Hence, $D$ is a $G$-domain. (2) Take a maximal independent subset $\left\{\alpha_{\lambda} ; \Lambda\right\}$ of $G$. Set $H=\sum_{\lambda} Z \alpha_{\lambda}$ and let $\mathfrak{P}$ be a $G$-ideal of $A_{G}[X]$. $\mathfrak{P} \cap A_{H}[X]=\mathfrak{P}^{\prime}$ is a $G$-ideal of $A_{H}[X]^{\lambda}$ by (1). Hence, $\mathfrak{P}^{\prime} \cap A=\mathfrak{P} \cap A$ is a $G$-ideal of $A$.

Proposition 16. Let $R$ be a ring. Assume $J$-rad. $(R /(\mathfrak{P} \cap R))=0$ imply $\mathrm{J}$-rad. $\left(R_{G}[X] / \mathfrak{P}\right)=0$ for every free $G$ and for every prime $\mathfrak{P}$ of $R_{G}[X]$. Then, $J$-rad. $(R / \mathfrak{P} \cap R)=0$ implies $J$-rad. $\left(R_{G}[X] / \mathfrak{P}\right)=0$ for any $G$ and for every prime $\mathfrak{P}$ of $R_{G}[X]$.

Proof. (1) If a domain $E$ is integral over a subdomain $D$ and if $\mathrm{J}-\mathrm{rad} . D$ is zero, then J-rad. $E$ is zero. (2) Consider a maximal independent subset $\left\{\alpha_{\lambda} ; \Lambda\right\}$ of $G$ and set $H=\sum_{\lambda} Z \alpha_{\lambda}$ and consider $R_{H}[X]$. The assertion follows from (1).

Propositon 17. Assume $G$ be finitely generated. Let $\mathfrak{B}$ be a prime ideal 
of $R_{G}[X]$. Then, if $J$-rad. $(R /(\mathfrak{P} \cap R))$ is zero, then $J-\operatorname{rad} .\left(R_{G}[X] / \mathfrak{P}\right)$ is zero.

Proof. We may suppose $G=Z$. Let $D$ be a domain, $\mathfrak{P}$ a prime ideal of $D[X]$ such that $\mathfrak{P} \cap D=0$. Assume $\mathrm{J}$-ad. $D$ be zero. We will show J-rad. $\left(D_{Z}[X] /\right.$ $\mathfrak{P})$ is zero. Suppose $\mathrm{J}$-rad. $\left(D_{Z}[X] / \mathfrak{P}\right) \neq 0$. There exists $g \bar{\in} \mathfrak{P}$ such that every maximal ideal of $D_{z}[X]$ containing $\mathfrak{P}$ contains $g$. We may suppose $o(g)=0$. By [26] $\S 3$, Th. 3, it follows $\mathfrak{P} \neq 0$. Set $n=\min \{\operatorname{deg} f ; 0 \neq f \in \mathfrak{P}, o(f)=0\}$ and take $0 \neq f \in \mathfrak{P}, o(f)=0$ such that $\operatorname{deg}(f)=n$. We introduce the order to $G$. $f=a_{0}$ $+a_{1} X+\cdots+a_{n} X^{n} . f$ is irreducible in $K[X]$. There exists $0 \neq b \in D$ such that $b \in(f, g) D[X]$. Let $m$ be a maximal ideal of $D$. Suppose $m \bar{\jmath} a_{0} a_{n}$. Suppose $m D_{z}[X]+\mathfrak{P}=D_{z}[X] . \quad 1=p(X)+m(X)$ for $p(X) \in \mathfrak{P}, m(X) \in m D_{z}[X] . \quad a_{n}^{i} p(X)$ $\in f(X) D_{z}[X]$ for some $i$. $a_{n}^{i}=f(X) h(X)+a_{n}^{i} m(X)$ for some $h(X) \in D_{z}[X]$. This is a contradiction. Hence, there exists a maximal ideal $\mathfrak{M}$ of $D_{z}[X]$ containing $m D_{z}[X]+\mathfrak{B} . \quad b \in \mathfrak{M} \cap D=\mathfrak{m}$. Hence, every maximal ideal of $D$ contains $a_{0} a_{n} b$.

Remark 18. Assume $G$ is free, $\mathfrak{P}$ a prime ideal of $A_{G}[X]$ and J-rad. ( $A$ / $(\mathfrak{P} \cap A))$ is zero. Then, J-rad. $\left(A_{G}[X] / \mathfrak{P}\right)$ need not be zero.

A Counter Example. $\quad A=k=$ countable fields, $G=\underline{Z \oplus_{N_{0}} \oplus \cdots}$. We confer the proof of Example 7.

\section{§7. $\boldsymbol{A}_{\mathbf{G}}[\mathbf{X}]_{\text {monics }}$}

Let $G$ be a totally ordered abelian group $(\neq 0)$. In this section we concern with the quotient ring $A_{G}[X]_{\text {monics. }}$.

Proposition 1. Let $D$ be a Dedekind domain, $S$ the set of monics of $D_{G}[X]$, where $G$ is a totally ordered abelian group $\neq 0$. If $D_{G}[X]_{s}$ is factorial, then $D$ is also factorial.

Proof. (1) Every element of $D$ is decomposed into a product of irreducible elements. (2) Every irreducible element $p$ of $D$ is an irreducible element of $D_{G}[X]_{s}$. (3) If $a, b \in D-\{0\}$ are associated in $D_{G}[X]_{s}$, then they are associated in $D$. (4) $D$ is factorial.

Proposition 2. Assume $G$ be totally ordered. Then $D_{G}[X]_{\text {monics }}$ is factorial if and only if $D$ is factorial.

Proof. The necessity. $D$ satisfies the maximal condition for principal ideals. The rest is similar with Prop. 1. The sufficiency. Similar with the proof of Prop. 12.

Proposition 3. Assume D be a Krull domain, G totally ordered of type 
$(0,0,0, \ldots)$. Then, canonically $C(D) \cong C\left(D_{G}[X]_{\text {monics }}\right)$.

Proof. By [24] Prop. 3.3, $D_{G}[X]$ is a Krull domain. By [24] Prop. 5.3, canonically $C(D) \cong C\left(D_{G}[X]\right)$. By Nagata theorem [28], we have the canonical onto homomorphism: $C(D) \rightarrow C\left(D_{G}[X]_{\text {monics }}\right)$. Let $\operatorname{cl}(\mathfrak{p}) \mapsto 0 . \quad \mathfrak{p} D_{G}[X]_{s}=$ $(f / g) D_{G}[X]_{s}$, where $S$ is the monics. Let $a$ be the coefficient of $f$ of the highest degree. Then, $\mathfrak{p}=a D$. Hence, $\mathrm{cl}(p)=0$.

Proposition 4. Assume $G$ be totally ordered abelian group $\neq 0, S$ the monics of $D_{G}[X]$ and assume $D_{G}[X]_{s}$ be a Krull domain. Then, $D$ is a Krull domain and canonically $C(D) \subseteq C\left(D_{G}[X]_{S}\right)$.

Proof. $\quad D_{G}[X]$ is a flat $D$-module ([26] $\$ 9$, Prop. 1). $D_{G}[X]_{S}$ is a faithfully flat $D$-module. Hence, $D$ is a Krull domain ([12] Prop. 6.10). There exists the canonical homomorphism: $C(D) \rightarrow C\left(D_{G}[X]_{s}\right)$. Let $\mathfrak{a}$ be a divisorial ideal of $D$ such that $a D_{G}[X]_{s}=f D_{G}[X]_{s}$ is principal. We may suppose $f \in D_{G}[X]$. Let $c$ be the coefficient of $f$ of the highest degree. Then, $\mathfrak{a}=c D$.

Lemma 5. Let $f, g \in D_{G}[X]$. Assume either the leading coefficient or the last coefficient of $g$ is a unit of $D$ and $g$ divide $f$ in $K_{G}[X]$. Then, $g$ divides $f$ in $D_{G}[X]$.

Proposition 6. Let $D$ be a Dedekind domain which is not a field. Assume $G$ be totally ordered of type $(0,0,0, \ldots)$. Let $S$ be the monics of $D_{G}[X]$. Then, every element of $C\left(D_{G}[X]_{S}\right)$ is of the form $\mathrm{cl}(\mathfrak{P})$, where $\mathfrak{B}$ is a proper prime ideal of $D_{G}[X]_{s}$.

Proof. Let $0 \neq a$ be a nonunit of $D, \pi \in G$ be of ht $(0,0,0, \ldots)$. Suppose $f g \in\left(1+a X^{\pi}\right) D_{G}[X]$ for $f, g \in D_{G}[X]$. By $\S 4$, Lem. $1,1+a X^{\pi}$ divides $f$ or $g$ in $K_{G}[X]$. By Lem. 5, $1+a X^{\pi}$ divides $f$ or $g$ in $D_{G}[X]$. Hence, $\left(1+a X^{\pi}\right)$ is a prime ideal of $D_{G}[X] . \quad \mathfrak{P}=\left(1+a X^{\pi}\right) D_{G}[X]_{s}$ is a proper prime ideal of $D_{G}[X]_{S}$ and $\operatorname{cl}(\mathfrak{P})=0$. Next, let $\operatorname{cl}(\mathfrak{H})$ be a nonzero element of $C\left(D_{G}[X]_{s}\right)$. By Prop. 3, there exists an ideal $a$ of $D$ such that $\operatorname{cl}\left(a D_{G}[X]_{s}\right)=\operatorname{cl}\left(\mathfrak{P I}^{-1}\right)$. There are $a, b$ such that $\mathfrak{a}=(a, b) . \quad \mathfrak{p}=\left(a+b X^{\pi}\right) K_{G}[X] \cap D_{G}[X]$ is a prime ideal of $D_{G}[X]$. By [26] §6, Prop. 10, $\mathfrak{p}=\left(a+b X^{\pi}\right) \mathfrak{a}^{-1} D_{G}[X] . \quad \operatorname{cl}\left(\mathfrak{p} D_{G}[X]_{s}\right)=\operatorname{cl}\left(\mathfrak{a}^{-1} D_{G}[X]_{s}\right)=$ $-\operatorname{cl}\left(\mathfrak{H}^{-1}\right)=\operatorname{cl}(\mathfrak{A})$.

Next, let $D$ be a local principal ideal domain, $G=Z \oplus \cdots \oplus Z$ with the lexicographic order, $S$ the monics of $D_{G}[X]$. Set $X^{\left(0, \ldots, 0, \frac{1}{i}, \ldots, 0\right)}=X_{i}(i \leq k-1)$, $X^{(0, \ldots, 0,1)}=Y$ and set $D_{G}[X]_{s}=D_{1}$. Generally, if $a, b$ of a domain do not have common divisors without units, we say $a, b$ are relatively prime.

Lemma 7. $F, G \in D\left[X_{1}, \ldots, X_{k-1}, Y\right]-\{0\} . \quad$ If $F, G$ are relatively prime in $D\left[X_{1}, \ldots, X_{k-1}, Y\right]$, then they are relatively prime in $D_{1}$. 
Lemma 8. Suppose $D_{1}$ is a PID for $k=1,2,3, \ldots, k-1$. Let $F, G \in D\left[X_{1}\right.$, $\left.\ldots, X_{k-1}, Y\right]$. Then, $(F, G) D_{1}$ is principal.

Proof. We express $X_{1}, \ldots, X_{k-1}$ by $X$.

$$
\begin{aligned}
& F=f_{0}+f_{1} Y+\cdots+f_{n} Y^{n}, \\
& G=g_{0}+g_{1} Y+\cdots+g_{m} Y^{m} ;
\end{aligned}
$$

$f_{i}, g_{j} \in D[X] ; f_{n} \neq 0 \neq g_{m}$. We prove by the induction on $n+m$. Suppose the proposition is $\mathrm{OK}$ till $\sigma-1$. Let $n+m=\sigma$. Suppose $(F, G) D_{1}$ is not principal. At first, $F \neq 0 \neq G$. Next, $f_{0} \neq 0$. Similarly, $g_{0} \neq 0$. Let $H$ be the GCD of $F, G$ in $D[X, Y] . \quad F=H F_{1}, G=H G_{1}$. By Lem. $7, F_{1}, G_{1}$ are relatively prime in $D_{1}$. $\left(F_{1}, G_{1}\right) D_{1}$ is not principal. $Y$-degree of $F_{1}$ is $n, Y$-degree of $G_{1}=m$.

$$
\begin{aligned}
& F_{1}=f_{0}^{1}+f_{1}^{1} Y+\cdots+f_{n}^{1} Y^{n}, \\
& G_{1}=g_{0}^{1}+g_{1}^{1} Y+\cdots+g_{m}^{1} Y^{m} ;
\end{aligned}
$$

$f_{i}^{1}, g_{j}^{1} \in D[X] . \quad f_{0}^{1} \neq 0 \neq g_{0}^{1}$. There exists a maximal ideal $\mathfrak{M} \supset\left(F_{1}, G_{1}\right) D_{1}$. Take an irreducible factor in $D[X, Y]$ of $F_{1}$ belonging to $\mathfrak{M}$. Say that $F_{2}$. Take an irreducible factor $G_{2}$ in $D[X, Y]$ of $G_{1}$ belonging to $\mathfrak{M}$. Let $n^{\prime}$ be the $Y$-degree of $F_{2}, m^{\prime}$ the $Y$-degree of $G_{2} ; n^{\prime} \leq n . \quad m^{\prime} \leq m$. If $n^{\prime}<n,\left(F_{2}, G_{2}\right) D_{1}$ is principal. $D_{1}=\left(F_{2}, G_{2}\right) D_{1} \in \mathfrak{M}$; a contradiction. Hence, $n^{\prime}=n$. Similarly, $m^{\prime}=m$.

$$
\begin{aligned}
& F_{2}=f_{0}^{2}+f_{1}^{2} Y+\cdots+f_{n}^{2} Y^{n}, \\
& G_{2}=g_{0}^{2}+g_{1}^{2} Y+\cdots+g_{m}^{2} Y^{m} ;
\end{aligned}
$$

$f_{i}^{2}, g_{j}^{2} \in D[X]$. We may suppose $n \leq m$. Suppose $G_{2} f_{n}^{2}-F_{2} g_{m}^{2} Y^{m-n}=0$. Then, $n=m, G_{2} f_{n}^{2}=F_{2} g_{n}^{2} . \quad F_{2}$ is associated with $f^{\prime} \in D[X]$ in $D[X, Y], G_{2}$ is associated with $g^{\prime} \in D[X]$ in $D[X, Y]$. Hence, $\left(F_{2}, G_{2}\right) D_{1}$ is principal, a contradiction. Hence, $G_{2} f_{n}^{2}-F_{2} g_{m}^{2} Y^{m-n} \neq 0$. Take an irreducible factor $H$ in $D[X, Y]$ of $G_{2} f_{n}^{2}$ $-F_{2} g_{m}^{2} Y^{m-n}$ belonging to $\mathfrak{M}$. $Y$-degree of $H$ is $\leq m-1$. Hence, $\left(F_{2}, H\right) D_{1}$ is principal. $\left(F_{2}, H\right) D_{1}=F_{2} D_{1}$. Hence, $F_{2}$ divides $f_{n}^{2}$ in $D_{1}$. Hence, $F_{2}$ is associated with $f^{\prime} \in D[X]$ in $D[X, Y]$. Suppose $g_{m}^{2} / f^{\prime} \in D[X]$. $g_{m}^{2}=f^{\prime} f^{\prime \prime}$ for $f^{\prime \prime} \in D[X]$. $Y$-degree of $G_{2}-f^{\prime} f^{\prime \prime} Y^{m}$ is $\leq m-1$. Hence, $\left(F_{2}, G_{2}-f^{\prime} f^{\prime \prime} Y^{m}\right) D_{1}$ is principal. $\left(F_{2}, G_{2}-f^{\prime} f^{\prime \prime} Y^{\prime \prime}\right) D_{1}=F_{2} D_{1}$; a contradiction. Hence, $g_{m}^{2} / f^{\prime} \bar{\epsilon} D[X]$. By Lem. 7, $1=f^{\prime}\left(f^{\prime \prime \prime} / h\right)+g_{m}^{2}\left(g^{\prime} / h\right)$ for $f^{\prime \prime \prime}, g^{\prime}, h \in D[X], h \in S . \quad G_{2} g^{\prime}+f^{\prime} f^{\prime \prime \prime} Y^{m} \in S . \quad$ A contradiction.

Proposition 9. In the above, $D_{G}[X]_{S}$ is a PID for every $k<\infty$.

Proof. Lem. 8.

Theorem 10. Let $D$ be a semilocal $P I D, G=Z \oplus \cdots \oplus Z$ with the lexicographic order, $S$ the monics of $D_{G}[X]$. Then, $D_{G}[X]_{S}$ is a PID. 
Proof. Similar with that of Prop. 9.

Proposition 11. Let $D$ be a domain. If $D_{G}[X]_{\text {monics }}$ is a PID for every finitely generated totally ordered $G$, then, $D_{G}[X]_{\text {monics }}$ is a PID for any totally ordered $G$.

Proof. Set $D_{1}=D_{G}[X]_{\text {monics. }}$ We show first that $D_{1}$ is bezout. Let $f, g \in D_{G}[X]$. Let $H$ be the ordered subgroup of $G$ generated by the powers of $f, g . \quad(f, g) D_{H}[X]_{\text {monics }}=h D_{H}[X]_{\text {monics }}$ for some $h \in D_{H}[X]$. Then, $(f, g) D_{1}$ $=h D_{1}$. Next, we show that $D_{1}$ is noetherian. Suppose $D_{1}$ is not noetherian. There exists a prime ideal $\mathfrak{P}$ of $D_{1}$ which is not finitely generated. Since $D_{z}[X]_{\text {monlcs }}$ is PID, $D$ satisfies the maximal condition on principal ideals. Hence, $D$ satisfies the maximal condition on principal ideals. Take an irreducible element $f \in \mathfrak{P}$. We may suppose $f \in D_{G}[X] . \quad f D_{1} \subsetneq \mathfrak{P}$. There is an irreducible element $g$ of $D_{1}$ such that $f D_{1} \bar{\exists} g \in \mathfrak{B} \cap D_{G}[X] . \quad(f, g) D_{1}=h D_{1}$ for some $h \in D_{G}[X] . \quad D_{1}=h D_{1}$ $=(f, g) D_{1} \subset \mathfrak{P}$. A contradiction.

Proposition 12. Let $G$ be totally ordered. Assume $D_{G}[X]_{\text {monics }}$ is a PID for every local PID D. Then, $D_{G}[X]_{\text {monics }}$ is a PID for any semi-local PID $D$.

Proof. Let $D$ be a semi-local PID. Set $D_{G}[X]_{\text {monics }}=D_{1}$. (1) At first, $D_{1}$ satisfies the maximal condition on principal ideals. (2) Next, $D_{1}$ is a factorial domain. Proof. Let $f$ be an irreducible element of $D_{1}$. We may suppose $f$ $\in D_{G}[X]$. Let $g h \in f D_{1}$ for $g, h \in D_{1}$. May suppose $g, h \in D_{G}[X]$. Then, $g$ or $h$ is divided by $f$. (3) $D_{1}$ is a bezout domain. Proof. Let $f, g \in D_{G}[X]$. May suppose $f, g \neq 0$. May suppose $f, g$ are relatively prime in $D_{1}$. Let $p_{1} D, \ldots, p_{n} D$ be the set of maximal ideals of $D . \quad\left(D_{p_{i}}\right)_{G}[X]_{\text {monics }}$ is a PID. Set $S_{i}=\left\{f \in D_{G}[X]\right.$; $p_{i}$ does not divide the terminal coefficient of $\left.f\right\} . \quad\left(D_{p_{i}}\right)_{G}[X]_{\text {monics }}=D_{G}[X]_{s_{i}}$. Since $f, g$ are relatively prime in $D_{G}[X]_{s_{i}},(f, g) D_{G}[X]_{s_{i}}=D_{G}[X]_{s_{i}} \quad f f_{i}+g g_{i}=h_{i}$ for $f_{i}, g_{i} \in D_{G}[X], h_{i} \in S_{i}$. May suppose the terminal coefficient of $h_{i}$ is divided by $p_{1}, p_{2}, \ldots, p_{i-1}, p_{i+1}, \ldots, p_{n}$. May suppose $\operatorname{deg} h_{1}=\operatorname{deg} h_{2}=\cdots=\operatorname{deg} h_{n}$. The terminal coefficient of $\sum h_{i}$ is a unit of $D$. $(f, g) D_{1}=D_{1}$. (4) $D_{1}$ is noetherian. Suppose $D_{1}$ is not noetherian. There exists a prime ideal $\mathfrak{P}$ of $D_{1}$ which is not finitely generated. Let $f \in D_{G}[X] \cap \mathfrak{P}$ be an irreducible element of $D_{1} . \quad f D_{1} \subsetneq \mathfrak{P}$. There exists an irreducible element $g$ of $D_{1}$ such that $f D_{1} \bar{\ni} g \in \mathfrak{P} \cap D_{G}[X] . \quad(f, g) D_{1}$ $=h D_{1} . \quad D_{1}=(f, g) D_{1} \subset \mathfrak{P}$; a contradiction. (5) $D_{1}$ is a PID.

EXAmple 13. $G=Z \oplus Z$ with the lexicographic order. Then, $Z_{G}[X]_{\text {monics }}$ is 1-dimensional.

Proof. $(1,0)<(0,1) . \quad X^{(1,0)}=X, X^{(0,1)}=Y$. Let $S^{\prime}$ be the monics of $Z[X]$. By $[13](40.6), D=Z[X]_{s^{\prime}}$ is a Dedekind domain. Let $S^{\prime \prime}$ be the monics 
of $D[Y] . \quad D[Y]_{s^{\prime \prime}}$ is also Dedekind. $D[Y]_{S^{\prime \prime}} \cong Z_{G}[X]_{\text {monics }}$.

Proposition 14. Let $D$ be a Dedekind domain with infinitely many maximal ideals. $\quad G=Z \oplus \cdots \oplus Z$ with the lexicographic order. Then, $D_{G}[X]_{\text {monics }}$ is 1-dimensional.

Proof. $\quad G(m)=Z \oplus \cdots \oplus Z$. Let $S(m)$ be the monics of $D_{G(m)}[X]$ and set $D_{G(m)}[X]_{S(m)}=D(m) . \quad B y[13](40.6), D(1)$ is 1-dimensional. Suppose $D\left(n^{\prime}\right)$ is 1-dimensional for $n^{\prime}<n . \quad D(n)=D_{G(n)}[X]_{S(n)}=D(n-1)[X]_{\text {monies }}$.

EXAMPLE 15. $G=\underbrace{Z \oplus \cdots}_{X_{0}}$ with the lexicographic order. Then, $Z_{G}[X]_{\text {monies }}$ is 1-dimensional.

Proof. Let $\mathfrak{P}$ be a ht 1 prime of $Z_{G}[X]_{\text {monics. }} \mathfrak{P}=f Z_{G}[X]_{\text {monies }}$ for $f \in Z_{G}[X]$. There exists $n$ such that $f \in Z_{H}[X]$ for $Z \oplus Z \oplus \cdots \oplus Z \oplus 0 \oplus 0 \oplus \cdots=H$. Set $0 \oplus \cdots \oplus 0 \oplus Z \oplus Z \oplus \cdots=K$ and set $Z_{H}[X]_{\text {monics }}=D$. By Prop. $14, D$ is 1dimensional. $f D=\mathfrak{p}$ is a maximal ideal of $D$. Set $D / \mathfrak{p}=k . \quad Z_{G}[X]_{\text {monics }} / \mathfrak{P}$ $=D_{K}[X]_{\text {monics }} / \mathfrak{p} D_{K}[X]_{\text {monics }}=k_{K}[X]_{\text {monies }}=$ field. Hence, $\mathfrak{P}$ is maximal.

EXAMPLe 16. Let $D$ be a Dedekind domain with infinitely many maximal ideals, $\Lambda$ a totally ordered set $\neq \phi, G_{\lambda}=Z, G=\underset{\lambda}{\oplus} G_{\lambda}$ with the lexicographic order. Then $D_{G}[X]_{\text {monics }}$ is 1-dimensional.

Proof. Let $\mathfrak{P}$ be a proper prime of $D_{G}[X]_{\text {monics. }}$ Take $D_{G}[X] \ni f \bar{E} \mathfrak{P}$. Take $\mathfrak{P} \cap D_{G}[X] \ni g \neq 0 . \quad f, g \in D_{G},[X]$ for a finite $\Lambda^{\prime} \subset \Lambda$ and $G^{\prime}=\oplus G_{\lambda}$. By Prop. 14, $\mathfrak{p}=\mathfrak{B} \cap D_{G^{\prime}}[X]_{\text {monics }}$ is a maximal ideal of $D_{G},[X]_{\text {monics. }} . \hat{f}_{1}-1 \in \mathfrak{p}$ for $f_{1} \in D_{G} \cdot[X]_{\text {monies }}$. Hence, $\mathfrak{P}$ is maximal.

Proposition 17. Let $D$ be a Dedekind domain with infinitely many maximal ideals. Assume $D_{G}[X]_{\text {monies }}$ is 1-dimensional for every finitely generated totally ordered $G$. Then, $D_{G}[X]_{\text {monies }}$ is 1-dimensional for any totally ordered G.

Proof. Let $\mathfrak{P}$ be a proper prime of $D_{G}[X]_{\text {monics. }}$ Take $\mathfrak{P} f \in D_{G}[X]$.

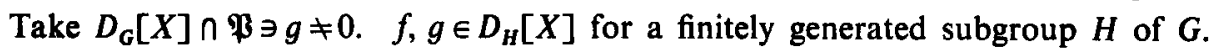
$\mathfrak{B} \cap D_{H}[X]_{\text {monics }}=\mathfrak{p}$ is a maximal ideal of $D_{H}[X]_{\text {monics }} f f_{1}-1 \in \mathfrak{p}$ for $f_{1} \in$ $D_{H}[X]_{\text {monics. }}$ Hence, $\mathfrak{P}$ is maximal.

Remark 18. Let $D$ be a Dedekind domain with class group cyclic of prime order. Assume every proper overring of $D_{G}[X]_{\text {monics }}$ is bezout for every finitely generated totally ordered $G$. Then every proper overring of $D_{G}[X]_{\text {monics }}$ is bezout for any totally ordered $G$. 


\section{§8. The factorial}

At first, on the HFD [43].

Proposition 1. If $D_{G}[X]$ is a HFD, then $D$ is a HFD.

Next, on the euclidean rings [41] Def. 1.

Proposition 2. $A_{G}[X]$ is a euclidean ring if and only if $G \cong Z$ and $A$ is a finite direct sum of fields.

Proof. The sufficiency. $\left(K_{i}\right)_{z}[X]$ is euclidean for fields $K_{i}$. Then, $\left(K_{1}\right)_{z}[X] \oplus \cdots \oplus\left(K_{n}\right)_{Z}[X]$ is also euclidean ([41] Prop. 6).

Proposition 3. Let $L / K$ be fields such that $K$ is algebraically closed in $L$. Then, every irreducible element of $K_{G}[X]$ is also irreducible in $L_{G}[X]$.

Proof. (1) $K\left(X_{1}, \ldots, X_{n}\right)$ is algebraically closed in $L\left(X_{1}, \ldots, X_{n}\right)$. (2) Every irreducible element of $K[X]$ is irreducible in $L[X]$ ([13] p. 410, 18). (3) Let $f$ be an irreducible element of $K\left[X_{1}, \ldots, X_{n}\right]$ such that $X_{n}$-degree of $f$ is $>0$. Then, $f$ is an irreducible element of $K\left(X_{1}, \ldots, X_{n-1}\right)\left[X_{n}\right]$. (4) If $f, g \in K\left[X_{1}, \ldots\right.$, $\left.X_{n}\right]$ are associated in $L\left[X_{1}, \ldots, X_{n}\right]$, then they are associated in $K\left[X_{1}, \ldots, X_{n}\right]$. (5) Every irreducible element $f=f\left(X_{1}, \ldots, X_{n}\right)$ of $K\left[X_{1}, \ldots, X_{n}\right]$ is irreducible in $L\left[X_{1}, \ldots, X_{n}\right]$. Suppose $f$ is properly decomposed in $L\left[X_{1}, \ldots, X_{n}\right]$ as $f=F G$. Suppose there exist $i$ such that $\operatorname{deg}_{X_{i}} F>0<\operatorname{deg}_{x_{i}} G$. May suppose $i=n . \quad f$ is an irreducible element of $K\left(X_{1}, \ldots, X_{n-1}\right)\left[X_{n}\right]$ by (3). Since $K\left(X_{1}, \ldots, X_{n-1}\right)$ is algebraically closed in $L\left(X_{1}, \ldots, X_{n-1}\right)((1)), f$ is an irreducible element of $L\left(X_{1}, \ldots\right.$, $\left.X_{n-1}\right)\left[X_{n}\right]((2))$. A contradiction. Hence, may suppose $F \in L\left[X_{1}, \ldots, X_{k}\right], G$ $\in L\left[X_{k+1}, X_{k+2}, \ldots, X_{n}\right] . \quad f=\sum h\left(e_{1}, \ldots, e_{n-k}\right) X_{k+1}^{e+1} \cdots X_{n}^{e_{n-k}}, h(\quad) \in K\left[X_{1}, \ldots, X_{k}\right]$. $b\left(e_{1}, \ldots, e_{n-k}\right) F=h\left(e_{1}, \ldots, e_{n-k}\right)$ for $b() \in L$. Hence, any two nonzero $h\left(e_{1}, \ldots\right.$, $\left.e_{n-k}\right), h\left(e_{1}^{\prime}, \ldots, e_{n-k}^{\prime}\right)$ are associated in $L\left[X_{1}, \ldots, X_{k}\right]$. By (4), they are associated in $K\left[X_{1}, \ldots, X_{k}\right] . \quad f$ is divided in $K\left[X_{1}, \ldots, X_{n}\right]$ by $h\left(e_{1}, \ldots, e_{n-k}\right)$. This is a contradiction. (6) Every irreducible element $f$ of $K_{G}[X]$ is irreducible in $L_{G}[X]$. Suppose $f=F G$ in $L_{G}[X]$ properly. Let $H$ be the subgroup generated by the powers of $f, F, G . \quad H=Z \alpha_{1} \oplus \cdots \oplus Z \alpha_{n} . \quad X^{\alpha_{i}}=X_{i} . \quad$ May suppose $\operatorname{ord}_{x_{i}} f=\operatorname{ord}_{x_{i}} F=$ ord $_{x_{i}} G=0 . \quad f$ is an irreducible element of $K\left[X_{1}, \ldots, X_{n}\right]$. By (5), $f$ is irreducible in $L\left[X_{1}, \ldots, X_{n}\right]$. A contradiction.

Proposition 4. Let $L / K$ be fields such that $K$ is algebraically closed in $L$. Then every prime element $f$ of $K_{G}[X]$ is a prime element of $L_{G}[X]$.

Proof. By Prop. $3, f$ is an irreducible clement of $L_{G}[X]$. Let $F G=f H$ for $F, G, H \in L_{G}[X]$. Let $G^{\prime}$ be the subgroup generated by the powers of $f, F, G$, 
$H$. Since $L_{G^{\prime}}[X]$ is factorial, $F \in f L_{G},[X]$ or $G \in f L_{G},[X]$. Hence, $f$ is prime in $L_{G}[X]$.

The next is on the Gaussian [13] p. 351, 7.

Proposition 5. If $A_{G}[X]$ is Gaussian, then $A$ is Gaussian.

Proposition 6. $D_{G}[X]$ is a Gaussian domain if and only if $D$ is a field and $G$ is isomorphic into $Q$.

Proof. [13] p. 351, 7(d) and [16].

Remark 7. Let $\left(A,{ }^{\prime}\right)$ be a local ring, then $A_{Z}[X]$ need not be Gaussian.

A Counter Example. $k=Z /(2), A=k[u], u^{2}=0 . \quad B=A_{Z}[X] . \quad B[Y] \ni$ $f=g=1+X+u Y . \quad A_{f g}=\left(1+X^{2}\right) B . \quad A_{f} A_{g}=\left(1+X^{2}, u+u X\right) B . \quad$ If $B$ is Gaussian, $u+u X \in\left(1+X^{2}\right) A_{z}[X] . \quad u+u X=\left(1+X^{2}\right)\left(a_{l} X^{l}+a_{l+1} X^{l+1}+\cdots+a_{h} X^{h}\right), a_{i}$ $\in A$. This is obviously imposaible.

Proposition 8. If $A_{G}[X]$ is Gaussian, $A$ is von Neumann regular and $G$ is isomorphic into $Q$.

Proof. Suppose $A$ has nonzero nilpotents. There exists $u \neq 0$ such that $u^{2}=0$. Take $0 \neq x \in G . \quad\left(A_{G}[X]\right)[Y] \ni f=1+X^{\alpha}+u Y, g=1+X^{\alpha}-u Y$. Then, $A_{f}=\left(1+X^{\alpha}, u\right)=A_{g}, A_{f} A_{g}=\left(\left(1+X^{\alpha}\right)^{2}, u+u X^{\alpha}\right), A_{f g}=\left(1+X^{\alpha}\right)^{2} . \quad u+u X^{\alpha}=(1+$ $\left.2 X^{\alpha}+X^{2 \alpha}\right) h$ for $h \in A_{G}[X]$. This is obviously impossible. Hence, $A$ is of zero nilradical. Let $\mathfrak{p}$ be a prime ideal of $A$. By Prop. $6, \mathfrak{p}$ is maximal and $G \hookrightarrow Q$, because $(A / \mathfrak{p})_{\mathrm{G}}[X]$ is Gaussian.

Theorem 9. $A_{6}[X]$ is Gaussian if and only if $A$ is von Neumann regular and $G$ is isomorphic into $Q$.

Proof. The sufficiency. At first, $\operatorname{dim} A=0$. Let $\mathfrak{M}$ be a maximal ideal of $A_{G}[X]$. Set $\mathfrak{M} \cap A=\mathfrak{p} . \quad \mathfrak{p}$ is a maximal ideal of $A$. By [13] p. 113,29, $A_{\mathfrak{p}}$ is a field. By Prop. 6, $\left(A_{\mathfrak{p}}\right)_{G}[X]$ is Gaussian. $\mathfrak{M N}^{\prime}=\{f / s ; f \in \mathfrak{M}, s \in A \backslash \mathfrak{p}\}$ is a maximal ideal of $\left(A_{\mathfrak{p}}\right)_{G}[X]$. By [13] p. 351, 7, $\left(A_{\mathfrak{p}}\right)_{G}[X]_{\mathfrak{M}^{\prime}}$ is Gaussian. $\left(A_{\mathfrak{p}}\right)_{G}[X]_{\mathfrak{M}^{\prime}}$ $\cong A_{G}[X]_{\Re}$. Hence, $A_{G}[X]$ is Gaussian.

If each element of a domain $D$ is a finite product of irreducible elements, $D$ is said to be atomic.

Proposition 10. If $D_{G}[X]$ is atomic, then $G$ is of type $(0,0,0, \ldots)$.

Proof. Let $0 \neq \alpha \in G$ and let $p$ be a prime number such that $\alpha$ is divided by every $p^{n}$. We will derive a contradiction. Let $1-X^{\alpha}=f_{1} \cdots f_{n}$ be an irreducible decomposition. Let $H$ be the subgroup generated by $\alpha$ and the powers of $f_{1}, \ldots, f_{n}$. $H=Z \pi \oplus Z \alpha_{1} \oplus \cdots \oplus Z \alpha_{k}, m \pi=\alpha$. Hence, may suppose $f_{i} \in D_{Z \pi}[X]$. May sup- 
pose $o\left(f_{i}\right)=0 . \quad 1-X^{\pi}$ divides some $f_{i}$ in $K_{Z_{\pi}}[X]$. By $\S 7$, Lem. 5, $1-X^{\pi}$ divides $f_{i}$ in $D_{Z_{\pi}}[X]$. $f_{i}=\left(1-X^{\pi}\right) g .1-X^{\pi}$ is reducible in $D_{G}[X]$. A contradiction. The rest is similar.

The next is on the Fossum's locally factorial [12].

Proposition 11. If $D_{G}[X]$ is locally factorial of Fossum, then $D$ is factorial.

Proposition 12. If $D_{G}[X]$ is Fossum's locally factorial, then $G$ is of type $(0,0,0, \ldots)$.

Proof. When $D$ is not a field. Take nonunit $0 \neq b$ of $D . D_{G}[X]_{b}$ $=\left(D_{b}\right)_{G}[X]$. By [15], $G$ is of type $(0,0,0, \ldots)$. When $D=K$ is a field. Let $0 \neq \alpha$ $\in G$ and $p$ a prime number. Suppose $p^{n+1} \alpha_{n+1}=\alpha$ is solvable for all $n$. $g=$ $1-X^{\alpha}+X^{2 \alpha}$. $\quad\left(1-X^{\alpha}\right) \subset\left(1-X^{\alpha / p}\right) \subset\left(1-X^{\alpha / p^{2}}\right) \subset \cdots$. Since $K_{G}[X]_{g}$ is factorial, $1-X^{\alpha / p^{n+1}}=\left(1-X^{\alpha / p^{n}}\right)\left(f / g^{m}\right)$ for some $n$ and $f \in K_{G}[X] . \quad g^{m}=(1+$ $\left.X^{\alpha / p^{n+1}}+X^{2 \alpha / p^{n+1}}+\cdots+X^{(p-1) \alpha / p^{n+1}}\right) f . \quad g, 1-X^{\alpha}$ have a nonunit common divisor. This is obviously impossible. The rest is similar.

Theorem 13. If $D_{G}[X]$ is Fossum's locally factorial, then $D_{G}[X]$ is factorial.

Proof. Prop. 11, Prop. 12, [15].

Proposition 14. Assume $D_{\mathrm{m}}$ is factorial for every maximal ideal $\mathrm{m}$ of $D, G$ be of type $(0,0,0, \ldots)$. Then, $D_{G}[X]_{\mathfrak{M}}$ is factorial for every maximal ideal $\mathfrak{M}$ of $D_{G}[X]$.

Proof. Set $\mathfrak{M} \cap D=\mathfrak{p}, D_{G}[X] \mid \mathfrak{M}=T$. Since $D_{\mathfrak{p}}$ is factorial, $\left(D_{\mathfrak{p}}\right)_{\mathfrak{G}}[X]$ is factorial. Hence, $\left(D_{\mathfrak{p}}\right)_{G}[X]_{T}$ is factorial. Hence, $D_{G}[X]_{\mathfrak{M}}$ is factorial.

Proposition 15. Assume $D_{G}[X]_{\mathbb{D}}$ be factorial for every maximal ideal IN of $D_{G}[X]$. Then, $D_{p}$ is factorial for every prime ideal $p$ of $D$.

Proof. Let $p$ be a prime ideal of $D . D_{G}[X]_{p D_{G}[X]}$ is factorial. $D_{G}[X]_{p D_{G}[X]}$ $=\left(D_{p}\right)_{G}(X)_{\text {loc }}$. By $\$ 13$, Prop. $4, D_{p}$ is factorial.

Proposition 16. (1) If $D$ is a factorial local domain, then $D_{G}(X)_{\text {loc }}$ is factorial. (2) Let $p$ be a prime ideal of $D$. Then, $D_{p}$ is factorial if and only if $D_{G}[X]_{p D_{G}[X]}$ is factorial.

Proof. (1) By $\$ 7$, Prop. 2, $D_{G}[X]_{\text {monics }}$ is factorial (G becomes totally ordered). $D_{G}(X)_{\text {loc }}$ is a quotient of $D_{G}[X]_{\text {monics. }}$ Hence, $D_{G}(X)_{\text {loc }}$ is factorial. (2) The necessity. By (1), $\left(D_{p}\right)_{G}[X]_{p(D p)_{G}[X]}$ is factorial. The sufficiency. §13, Prop. 4. 
Remark 17. Assume $Q_{G}[X]_{M}$ be factorial for every maximal ideal $M$ of $Q_{G}[X]$, then $G$ need not be of type $(0,0,0, \ldots)$.

A Counter Example. $G$ in $\$ 10$, Remark 3.

\section{\$9. The chain conditions}

At first, on the saturated chain conditions ([13] p. 383).

Proposition 1. If $A_{G}[X]$ satisfies the saturated chain condition, $A$ satisfies the saturated chain condition.

Proposition 2. $A_{G}[X]$ satisfies the saturated chain condition if and only if $(A / \mathfrak{p})_{G}[X]$ satisfies the saturated chain condition for every minimal prime ideal $\mathfrak{p}$ of $A$.

The next is on the catenary ([20] p. 23).

Proposition 3. If $A_{G}[X]$ is catenary, then $A$ is catenary.

The next is on the chain condition for prime ideals in noetherian domains [45] p. 326.

Proposition 4. If the chain condition for prime ideals in noetherian $D_{G}[X]$ holds, then so is in $D$.

Proof. Similar with that of Prop. 7.

The next is on the H-domain ([38]).

Remark 5. Even if $D_{G}[X]$ is an H-domain, $D$ need not be an H-domain.

A Counter Example. Take a non-H-domain $D$. $G=G_{1} \oplus G_{2} \oplus G_{3} \oplus \cdots$, $G_{i}=Z$. $\operatorname{dim} D_{G}[X]=\infty$. Let $\mathfrak{P}$ be a ht 1 prime of $D_{G}[X]$ and set $\mathfrak{p}=D \cap \mathfrak{P}$. When $\mathfrak{p} \neq 0 . \quad D_{G}[X] / \mathfrak{P} \cong(D / \mathfrak{p})_{G}[X] . \quad \operatorname{dim} D_{G}[X]=\operatorname{dim} D_{G}[X] / \mathfrak{P}+1 . \quad$ Next, the case $D \cap \mathfrak{P}=0$. Take an irreducible element $f$ of $K_{G}[X]$ belonging to $\mathfrak{P}$. Let $h \in D_{G}[X] \cap f K_{G}[X]$. Then, $D_{G}[X] \cap f K_{G}[X]=\mathfrak{P}$. Set $X^{\left(0, \ldots 0, \frac{1}{i}, \ldots\right)}=X_{i}$. $D_{G}[X]=D_{Z}\left[X_{1}, X_{2}, \ldots\right]$. May suppose $f \in D\left[X_{1}, \ldots, X_{n}\right]$. Set $\left(\mathfrak{P}, 1-X_{n+1}\right.$, $\left.1-X_{n+2}, \ldots, 1-X_{n+m}\right)=\mathfrak{P}_{m}$. Then, $\mathfrak{P}_{m}$ is a prime ideal of $D_{z}\left[X_{1}, X_{2}, \ldots\right]$. Hence, $\operatorname{dim} D_{G}[X] / \mathfrak{P}=\infty . \operatorname{dim} D_{G}[X]=\operatorname{dim} D_{G}[X] / \mathfrak{P}+1$. Hence, $D_{G}[X]$ is an $\mathrm{H}$ domain.

The next is on the equidimensional $[20](0,16.1 .4)$.

Remark 6. Even if $A_{G}[X]$ is equidimensional, $A$ need not be equidimensional.

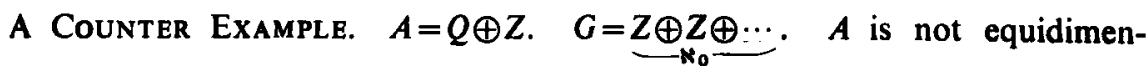


sional. Let $\mathfrak{B}$ be a minimal prime ideal of $A_{G}[X] . \quad \mathfrak{p}=\mathfrak{B} \cap A$ is a minimal prime of $A . \quad \mathfrak{P}=\mathfrak{p} A_{G}[X] . \quad \operatorname{dim} A_{G}[X] / \mathfrak{p} A_{G}[X]=\infty$. Hence, $A_{G}[X]$ is equidimensional.

The next is on the biequidimensional $[20](0,16.1 .4)$.

Proposition 7. If $A_{G}[X]$ is noetherian and biequidimensional, then so is $A$.

Proof. We may suppose $G=Z$. $\infty>n=\operatorname{dim} A_{Z}[X]$. Suppose $A$ is not biequidimensional. There exist two maximal chains of prime ideals containing $1-X: \mathfrak{P}_{0} \supsetneq \mathfrak{P}_{1} \supsetneq \cdots \supsetneqq \mathfrak{P}_{k}, \mathfrak{Q}_{0} \supsetneq \mathbb{Q}_{1} \supsetneq \cdots \supsetneq \mathfrak{Q}_{1}$ such that $k \neq 1$. Set $\mathfrak{P}_{k} \cap A=\mathfrak{p}$. If $\mathfrak{P}_{k}$ is a minimal prime of $A_{z}[X], \mathfrak{P}_{k}=\mathfrak{p} A_{z}[X]$. It follows $\mathfrak{p} \ni 1$; a contradiction. Hence, there exists a prime ideal $\mathfrak{P}_{k+1} \subset \mathfrak{P}_{k}$. By the altitude theorem of Krull ([29] (9.3)), ht $\mathfrak{P}_{k} \leq 1$. Hence, $\operatorname{dim} A_{Z}[X]=k+1$. Similarly, $\operatorname{dim} A_{Z}[X]=l+1$. Hence, $k+1=l+1$; a contradiction.

Proposition 8. If $A_{G}[X]$ satisfies the chain condition for primes of [29], then so does $A$.

The next is on the dimension formula [45].

Proposition 9. Consider the proposition, "if $D_{G}\left[X_{1}, \ldots, X_{n}\right]$ satisfies the chain condition for primes of [45] for every $n$, then $D$ satisfies the dimension formula". If the proposition holds for $G=Z$, then the proposition holds generally.

Proof. Prop. 4.

Proposition 10. If the dimension formula for noetherian $D_{G}[X]$ holds, then also holds for $D$.

Proof. (1) For every noetherian local domain $(D, \mathfrak{p})$, ht $\left(\mathfrak{p} D_{\mathbf{z}}[X]\right)=\mathrm{ht} \mathfrak{p}$. (2) Let $\mathfrak{p}$ be a prime ideal in a noetherian domain $D$. Then $h t\left(\mathfrak{p} D_{Z}[X]\right)=h t p$. (3) Proof of Prop. 10. By (2).

THEOREM 11. The dimension formula for noetherian $D_{G}[X]$ holds if and only if it holds for the noetherian $D$.

Proof. The sufficiency. We may suppose $G=Z$.

The next is on the taut [39].

REMARK 12. Even if $A_{Z}[X]$ is taut, $A$ need not be taut.

A Counter E xample. $A=Q \oplus Z$.

Remark 13. Assume $\operatorname{dim} k_{G}[X]=1$. Then, $k_{G}[X]$ need not be finitely generated ring extension over $k$. 
A Counter Example. $G=Q$.

Proposition 14. If $\operatorname{dim} k_{G}[X]=1, G$ is of type $(0,0,0, \ldots)$, then $k_{G}[X]$ is finitely generated ring extension over $k$.

Proof. $\left(1-X^{\alpha} ; \alpha \neq 0\right)$ is a prime ideal of $k_{G}[X]$. Let $\pi$ be of ht $(0,0,0, \ldots)$. $1-X^{\pi}$ is a prime element of $k_{G}[X]([24](2.2))$. Hence, $\left(1-X^{\alpha} ; \alpha \neq 0\right)=\left(1-X^{\pi}\right)$. Hence, $G=Z \pi . \quad k_{G}[X]=k\left[X^{\pi}, X^{-\pi}\right]$.

Proposition 15. $k_{G}[X]$ is 1-dimensional if and only if $G \hookrightarrow Q$.

Proposition 16. Let $A$ be noetherian. Then, $\operatorname{dim} A_{G}[X]=\operatorname{dim} A+$ torsionfree rank of $G$.

Proof. Confer $\$ 3$, Lem. 10.

\section{§10. The finite characters}

If $D_{\mathrm{p}}$ is a Krull domain for every prime ideal $p$ of $D, D$ is said to be an almost Krull domain.

Proposition 1. If $D_{G}[X]$ is almost Krull, then so is $D$.

Proposition 2. If $D$ is almost Krull, $G$ of the type $(0,0,0, \ldots)$, then $D_{G}[X]$ is almost Krull.

Proof. Let $\mathfrak{P}$ be a prime ideal of $D_{G}[X], \mathfrak{P} \cap D=\mathfrak{p}, D_{G}[X] \mid \mathfrak{P}=M$. $\left(D_{p}\right)_{G}[X]$ is a Krull domain $([24](3.3))$. Hence, $D_{G}[X]_{\mathfrak{R}}=\left(D_{\mathrm{p}}\right)_{G}[X]_{M}$ is a Krull domain.

RemarK 3. Even if $Q_{G}[X]$ is almost Krull, $G$ need not be of type $(0,0,0, \ldots)$.

A Counter Example. $G=\left\{b / 2^{a} ; a, b \in Z\right\}$. If $\mathfrak{P}$ is a nonzero prime of $Q_{G}[X], Q_{G}[X]_{\mathfrak{B}}$ is a regular local ring.

The next is on the generalized Krull domain ([13] p. 524).

Proposition 4. If $D$ is a generalized Krull domain, $G$ of type $(0,0,0, \ldots)$, then $D_{G}[X]$ is a generalized Krull domain.

Proof. Let $\left\{v_{\lambda} ; A\right\}$ be a family of valuations on $K$ defining the generalized Krullness of $D$. Let $v_{\lambda}^{\prime}$ be the trivial extension of $v_{\lambda}$ to $K_{G}(X)$. Let $\left\{f_{\sigma} ; \Sigma\right\}$ be a complete representatives of mutually non-associated irreducible elements of $K_{G}[X]$. Let $w_{\sigma}^{\prime}$ be the $f_{\sigma}$-adic valuation on $K_{G}(X)$. Then, $\left\{v_{\alpha}^{\prime}, w_{\sigma}^{\prime} ; \Lambda, \Sigma\right\}$ defines the generalized Krullness of $D_{G}[X]$.

Proposition 5. If $D_{G}[X]$ is a generalized Krull domain, then so is $D$. 
Remark 6. Even if $D_{G}[X]$ is a generalized Krull domain, $G$ need not be of the type $(0,0,0, \ldots)$.

A Counter Example. $D=K=Z /(p) . \quad G=\left\{a / p^{n} ; a, n \in Z\right\}$. Let $\left\{f_{\lambda} ; \Lambda\right\}$ be the complete representatives of mutually non-associated irreducible elements of $K_{Z}[X]$. Let $v_{\lambda}$ be the $f_{\lambda}$-adic valuation on $K(X)$. We extend $v_{\lambda}$ to $V_{\lambda}$ of $K_{G}(X)$ canonically. $\left\{V_{\lambda} ; \Lambda\right\}$ determines the generalized Krullness of $D_{G}[X]$.

Proposition 7. Assume the characteristic of $D$ is zero. Then, $D_{G}[X]$ is a generalized Krull domain if and only if $D$ is a generalized Krull domain and $G$ is of the type $(0,0,0, \ldots)$.

Proof. The necessity. $K_{G}[X]$ is also a generalized Krull domain ([13] (43.6)). Let $p_{1}<p_{2}<p_{3}<\cdots$ be a sequence of prime numbers and $0 \neq \alpha \in G$ such that $p_{i}$ divides $\alpha$. Let $\left\{v_{\lambda} ; \Lambda\right\}$ be the defining family of valuations of $K_{G}[X]$. The number $n$ of the $v_{\lambda}$ such that $v_{\lambda}\left(1-X^{\alpha}\right)>0$ is finite. It is impossible that $v_{\lambda}\left(1-X^{\alpha / p_{1}}\right)>0, v_{\lambda}\left(1+X^{\alpha / p_{1}}+X^{2 \alpha / p_{1}}+\cdots+X^{\left(p_{1}-1\right) \alpha / p_{1}}\right)>0$. Hence, the number of the $v_{\lambda}$ such that $v_{\lambda}\left(1-X^{\alpha / p_{1}}\right)>0$ is at most $n-1$. Like this, we see that $1-X^{\alpha /\left(p_{1} \cdots p_{n}\right)}$ is a unit of $K_{G}[X]$. This is a contradiction. The rest is similar.

The next is on the Krull type ([13] p. 537).

Proposition 8. If $D$ is Krull type, $G$ of type $(0,0,0, \ldots)$, then $D_{G}[X]$ is of Krull type.

Proposition 9. If $D_{G}[X]$ is Krull type, then $D$ is Krull type.

Proof. Let $\left\{v_{\lambda} ; \Lambda\right\}$ be the defining family of valuations on $D_{G}[X]$. Then, $\left\{v_{\lambda} \mid K ; A\right\}$ defines the Krull type of $D$.

Remark 10 . Even if $D$ is Krull type, $D_{G}[X]$ need not be Krull type.

A Counter Example. $D=Z$ or $D=Q . \quad G=Q$. Suppose $D_{G}[X]$ is Krull type. $\left\{v_{\lambda} ; A\right\}$ is the defining family. The number of the $v_{\lambda}$ such that $v_{\lambda}(1-X)>0$ is finite $n$. The number of the $v_{\lambda}$ such that $v_{\lambda}\left(1-X^{1 / 2}\right)>0$ is at most $n-1$. Like this, the number of the $v_{\lambda}$ such that $v_{\lambda}\left(1-X^{1 / 2^{n}}\right)$ is zero. Hence, $1-X^{1 / 2^{n}}$ is a unit. This is impossible.

Proposition 11. Assume the characteristic of $D$ is 0 . Then, $D_{G}[X]$ is Krull type if and only if $D$ is Krull type and $G$ is of type $(0,0,0, \ldots)$.

Proof. Prop. 8, Prop. 9, [13] p. 537.

The next is on the finite character of rational type [6] $\$ 1$, exerc. 28 ).

Proposition 12. If $D_{G}[X]$ is of finite character of rational type, then so is 
D.

Proposition 13. If $D$ is of finite character of rational type, $G$ of type $(0,0,0, \ldots)$, then $D_{G}[X]$ is of finite character of rational type.

Proposition 14. Let the characteristic of $D$ is zero. Then, $D_{G}[X]$ is of finite character of rational type if and only if $D$ is of finite character of rational type and $G$ is of type $(0,0,0, \ldots)$.

Proof. The necessity. By Prop. 12, $D$ is of finite character of rational type. $K_{G}[X]$ is also of finite character of rational type ([13] (43.6)). The rest is similar with that of Prop. 7.

The next is on the finite real character [13] p. 524 .

Proposition 15. If $D_{G}[X]$ is of finite real character, then so is $D$.

Proposition 16. If $D$ is of finite real character and $G$ of type $(0,0,0, \ldots)$, then $D_{G}[X]$ is of finite real character.

Proposition 17. Let the characteristic is zero. Then, $D_{G}[X]$ is of finite real character if and only if so is $D$ and $G$ be of type $(0,0,0, .$.$) .$

Proof. The sufficiency follows from Prop. 16.

The next is on the $K$ domains ([35] DEF. 1.1).

Proposition 18. If $D_{G}[X]$ is a $K$ domain, then so is $D$.

Proof. Let $\left\{v_{\lambda}^{\prime} ; \Lambda\right\}$ be the defining family of valuations of $D_{G}[X]$. Set $v_{\lambda}^{\prime} \mid K=v_{\lambda}$. We will show that the center $P(v)$ of $v=v_{\lambda}^{\prime} \mid K$ on $D$ is divisorial. Set $v_{\lambda}^{\prime}=v^{\prime} . \quad P\left(v^{\prime}\right)=\bigcap_{M}\left(f_{\mu} / g_{\mu}\right) D_{G}[X]$. Take $0 \neq a \in P(v) . \quad a=\left(f_{\mu} / g_{\mu}\right) h_{\mu}$ for some $h_{\mu}$. $P\left(v^{\prime}\right)=\bigcap_{M}\left(a / h_{\mu}\right) D_{G}^{M}[X] . \quad h_{\mu}=\sum b_{\mu \alpha} X^{\alpha}$. Then, $P(v)=P\left(v^{\prime}\right) \cap D=\underset{0 \neq b_{\mu \alpha}}{\bigcap}\left(a / b_{\mu \alpha}\right) D$.

Proposition 19. Assume $D$ be a $K$ domain, $G$ of type $(0,0,0, \ldots)$. Then, $D_{G}[X]$ is a $K$ domain.

Proof. Let $\left\{v_{\lambda} ; \Lambda\right\}$ be the defining family of valuations of $D$. Let $w_{\lambda}$ be the trivial extension of $v_{\lambda}$ to $K_{G}(X)$. Let $\left\{f_{\sigma} ; \Sigma\right\}$ be the complete representatives of mutually non-associated irreducible elements of $K_{G}[X]$ and let $u_{\sigma}$ be the $f_{\sigma}$-adic valuation. Let $W_{\lambda}, U_{\sigma}$ be the valuation rings of $w_{\lambda}, u_{\sigma}$ respectively. Then, we see that $\bigcap_{\lambda_{0} \neq \lambda} W_{\lambda} \cap U_{\sigma} \not \subset W_{\lambda_{0}}, \bigcap_{\lambda} W_{\lambda} \bigcap_{\sigma 0 \neq \sigma} U_{\sigma} \not \subset U_{\sigma_{0}}$. By [35] Prop. 1.7, $D_{G}[X]$ is a $\mathrm{K}$ domain.

EXAmple 20. $Q_{Q}[X]$ is not a $\mathrm{K}$ domain.

Proof. If $Q_{Q}[X]$ is a $\mathrm{K}$ domain, $Q_{Q}[X]$ becomes a Dedekind domain ([33], [34] p. 433). This is impossible. 
The next is on the finite character ([13] p. 524).

Proposition 21. If $D_{G}[X]$ is of finite character, then so is $D$.

Proposition 22. If $D$ is of finite character, $G$ of type $(0,0,0, \ldots)$, then $D_{G}[X]$ is of finite character.

Proposition 23. Assume the characteristic of $D$ is zero. Then, $D_{G}[X]$ is of finite character if and only if so is $D$ and $G$ is of type $(0,0,0, \ldots)$.

Proof. Prop. 22, Prop. 21, [13] (43.6).

Proposition 24. Assume the characteristic of $D$ is $p>0, D_{G}[X]$ be of finite character, $G \ni \alpha \neq 0, q$ a prime number $\neq p$. Then,

(1) An infinite number of prime numbers do not divide $\alpha$.

(2) $q^{n}$ does not divide a for some $n$.

Proof. A little modification of the proof of Prop. 7.

The next is on the Krull ring [22].

Proposition 25. If $A_{G}[X]$ is a Krull ring, then $A$ is a Krull ring or the total quotient ring of $A$ is $A$.

Remark 26. Even if $A$ is a Krull ring, $A_{Z}[X]$ need not be a Krull ring.

A Counter Example. $A=Z \oplus Z /(4)$.

\section{\$11. The r-noetherian}

Lemma 1. Assume $G$ be finitely generated and $G \supset\left\{\alpha_{1}, \ldots, \alpha_{n+1}\right\}$ is in-

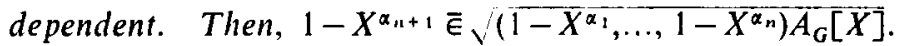

Proof. There exist $m_{1}>0$ and a ht $(0,0,0, \ldots)$ element $\beta_{1}$ such that $m_{1} \beta_{1}$ $=\alpha_{1} . \quad\left\{\beta_{1}, \alpha_{2}, \ldots, \alpha_{n+1}\right\}$ is independent. $\left(1-X^{\alpha_{1}}, 1-X^{\alpha_{2}}, \ldots, 1-X^{\alpha_{n}}\right) \subset\left(1-X^{\beta_{1}}\right.$, $\left.1-X^{\alpha_{2}}, \ldots, 1-X^{\alpha_{n}}\right)$. By $[24](2.1), Z \beta_{1}$ is a direct summand of $G=H_{1} ; G=$ $Z \beta_{1} \oplus H_{2}$. Next, $\alpha_{2}=m_{21} \beta_{1}+m_{22} \beta_{2}, \beta_{2}$ is a ht $(0,0,0, \ldots)$ element of $H_{2}, m_{i j}$ $\in Z . \quad\left\{\beta_{1}, \beta_{2}, \alpha_{3}, \ldots, \alpha_{n+1}\right\}$ is independent. $\left(1-X^{\beta_{1}}, 1-X^{a_{2}}, \ldots, 1-X^{\alpha_{n}}\right) \subset(1-$ $\left.X^{\beta_{1}}, 1-X^{\beta_{2}}, 1-X^{\alpha_{3}}, \ldots, 1-X^{\alpha_{n}}\right) . \quad G=Z \beta_{1} \oplus Z \beta_{2} \oplus H_{3} . \quad$ Next, $\quad \alpha_{3}=m_{31} \beta_{1}+$ $m_{32} \beta_{2}+m_{33} \beta_{3}, \beta_{3}$ is a ht $(0,0,0, \ldots)$ element of $H_{3}$. $\left\{\beta_{1}, \beta_{2}, \beta_{3}, \alpha_{4}, \ldots, \alpha_{n+1}\right\}$ is independent. $\left(1-X^{\beta_{1}}, 1-X^{\beta_{2}}, 1-X^{\alpha_{3}}, \ldots, 1-X^{\alpha_{n}}\right) \subset\left(1-X^{\beta_{1}}, 1-X^{\beta_{2}}, 1-\right.$ $\left.X^{\beta_{3}}, 1-X^{\alpha_{4}}, \ldots, 1-X^{\alpha_{n}}\right) . \quad G=Z \beta_{1} \oplus Z \beta_{2} \oplus Z \beta_{3} \oplus H_{4}$. Continue this process. In $n$-times, we have $\alpha_{n}=m_{n 1} \beta_{1}+m_{n 2} \beta_{2}+\cdots+m_{n n} \beta_{n}, \beta_{n}$ is a ht $(0,0,0, \ldots)$ element of $H_{n} . \quad\left\{\beta_{1}, \ldots, \beta_{n}, \alpha_{n+1}\right\}$ is independent.

$$
\left(1-X^{\beta_{1}}, \ldots, 1-X^{\beta_{n-1}}, 1-X^{\alpha_{n}}\right) \subset\left(1-X^{\beta_{1}}, \ldots, 1-X^{\beta_{n-1}}, 1-X^{\beta_{n}}\right) .
$$




$$
G=Z \beta_{1} \oplus \cdots \oplus Z \beta_{n} \oplus H_{n+1} .
$$

Next, $\alpha_{n+1}=m_{n+1,1} \beta_{1}+\cdots+m_{n+1, n+1} \beta_{n+1}, \beta_{n+1}$ is a ht $(0,0,0, \ldots)$ element of $H_{n+1}$.

$$
\begin{aligned}
G & =Z \beta_{1} \oplus \cdots \oplus Z \beta_{n+1} \oplus H_{n+2} \\
& =Z \beta_{1} \oplus \cdots \oplus Z \beta_{n+1} \oplus Z \beta_{n+2} \oplus \cdots \oplus Z \beta_{n+l} .
\end{aligned}
$$

Suppose $1-X^{\alpha_{n+1}} \in \sqrt{\left(1-X^{\alpha_{1}}\right.}, \ldots, \overline{\left.1-X^{\alpha_{n}}\right)}$. For some $k>0$,

A Must

$$
\left(1-X^{\alpha_{n+1}}\right)^{k} \in\left(1-X^{\alpha_{1}}, \ldots, 1-X^{\alpha_{n}}\right) \subset\left(1-X^{\beta_{1}}, \ldots, 1-X^{\beta_{n}}\right) . \quad \cdots
$$

It follows $\left(1-X^{m_{n+1}, n+1 \beta_{n+1}}\right)^{k} \in\left(1-X^{\beta_{1}}, \ldots, 1-X^{\beta_{n}}\right)$. Setting $X^{\beta_{i}}=X_{i},(1-$ $\left.X_{n+1}^{m_{n+1, n+1}}\right)^{k}=\left(1-X_{1}\right) g_{1}+\cdots+\left(1-X_{n}\right) g_{n}$ for $g_{i} \in A_{Z}\left[X_{1}, \ldots, X_{n}\right]$. This is obviously impossible.

Lemma 2. Assume $G \supset\left\{\alpha_{1}, \ldots, \alpha_{n+1}\right\}$ is independent. Then, in $A_{G}[X]$, $1-X^{x_{n+1}} \bar{E}_{\sqrt{ }}\left(\overline{I-X^{\alpha_{1}}}, \ldots, 1-X^{\left.\alpha_{n}\right)}\right.$.

Proof. Reduce to the case where $G$ is finitely generated.

The next is on the noetherian spectrum $[19](0,2.2 .1)$.

Proposition 3. If $\operatorname{Spec}\left(A_{G}[X]\right)$ is noetherian, then $G$ is of finite torsionfree rank.

Proof. Suppose the contrary. There exists $G \supset\left\{\alpha_{1}, \alpha_{2}, \alpha_{3}, \ldots\right\}$ which is independent. $\quad \mathscr{I}_{n}=\left(1-X^{\alpha_{1}}, \ldots, 1-X^{\alpha_{n}}\right)$. By Lem. 2, it follows $V^{\prime}\left(\mathscr{P}_{1}\right) \supsetneqq V\left(\mathscr{P}_{2}\right)$ $\supsetneq \cdots$.

Proposition 4. If $\operatorname{Spec}\left(A_{G}[X]\right)$ is noetherian, then $\operatorname{Spec}(A)$ is noetherian.

Remark 5. Even if $\operatorname{Spec}(A)$ is noetherian, $\operatorname{Spec}\left(A_{Q}[X]\right)$ need not be so.

A Counter Example. $A=k$, ch. $k=0 . \quad k_{Q}[X] \supset, 1-\overline{X^{1 / 2}}=\mathfrak{Q I}_{i} . \quad \mathfrak{H I}_{i} \subset$ $\mathfrak{H}_{i+1}$. Suppose $\mathfrak{V}_{i}=\mathfrak{V I}_{j}, i<j . \quad 1-X^{1 / 2^{i+1}} \in \sqrt{1-X^{1 / 2}} \cdot \quad\left(1-X^{1 / 2^{i+1}}\right)=(1-$ $\left.X^{1 / 2 i}\right) f . \quad\left(1-X^{1 / 2^{i+1}}\right)^{n-1}=\left(1+X^{1 / 2^{i+1}}\right) f . \quad Y=X^{1 / m}$ for some $m . \quad\left(1-Y^{a}\right)^{n-1}$ $=\left(1+Y^{a}\right) g(Y), g(Y) \in k\left[Y^{a}, Y^{-a}\right]$. This is impossible.

An ideal $\mathfrak{a}$ of $A$ is said to be a regular ideal if $\mathfrak{a}$ contains a non-zerodivisor of $A$. If $A$ satisfies the maximal condition on regular ideals, $A$ is said to be r-noetherian.

Proposition 6. If $A_{G}[X]$ is r-noetherian, the torsion-free rank of $G$ is finite.

Proof. If $G \supset\left\{\alpha_{1}, \alpha_{2}, \alpha_{3}, \ldots\right\}$ is independent, set $\left(1-X^{\alpha}, \ldots, 1-X^{\alpha_{n}}\right)=$ $\mathfrak{a}_{n}$. By Lem. 2, $\mathfrak{a}_{n} \subsetneq \mathfrak{a}_{n+1}$. 
Lemma 7. Let $H$ be a finitely generated subgroup of $G, H=Z \alpha_{1} \oplus \cdots \oplus$ $Z \alpha_{n}$. Then, $\left(1-X^{\alpha_{1}}, \ldots, 1-X^{\alpha_{n}}\right)$ does not depend on $\alpha_{1}, \ldots, \alpha_{n}$.

We set $\left(1-X^{\alpha_{1}}, \ldots, 1-X^{\alpha_{n}}\right)=\mathfrak{a}_{H, G}$.

LemMA 8. Let $H, K$ be finitely generated subgroups of $G$ such that $H \subset K$. Then, $a_{H, G} \subset a_{K, G}$. Let $G^{\prime}$ be a subgroup such that $H \subset K \subset G^{\prime}$ and $a_{H, G^{\prime}}=a_{K, G^{\prime}}$, then $\mathfrak{a}_{H, G}=\mathfrak{a}_{K, G}$.

Lemma 9. Let $G$ be finitely generated of the torsion-free rank $n, H, K$ be subgroups of the torsion-free rank $n$ such that $H \subsetneq K$. Then, $\mathfrak{a}_{H, G} \subsetneq a_{K, G}$.

Proof. If we take suitable basis $\left\{\gamma_{1}^{\prime}, \ldots, \gamma_{n}^{\prime}\right\},\left\{\beta_{1}^{\prime}, \ldots, \beta_{n}^{\prime}\right\}$ of $G, K$ respectively, $\beta_{i}^{\prime}=e_{i}^{\prime} \gamma_{i}^{\prime}, 0<e_{i}^{\prime}$. Take a base $\left\{\alpha_{1}, \ldots, \alpha_{n}\right\}$ of $H$ and a permutation $\sigma$ such that

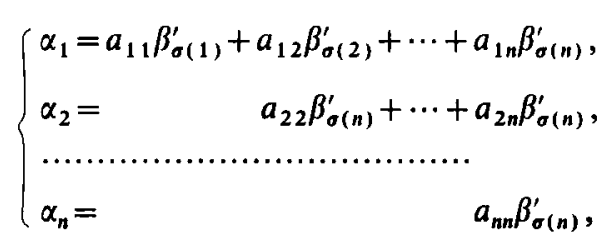

$1 \leq a_{11} \leq a_{22} \leq \cdots \leq a_{n n}$. Then, $a_{n n}>1$. Let $k=\min \left\{i ; a_{i i}>1\right\}$. Set $\theta_{1}^{\prime}=\alpha_{1}, \cdots$, $\theta_{k}^{\prime}=\alpha_{k}, \theta_{k+1}^{\prime}=\beta_{\sigma(k+1)}^{\prime}, \ldots, \theta_{n}^{\prime}=\beta_{\sigma(n)}^{\prime} ; E=Z \theta_{1}^{\prime}+\cdots+Z \theta_{n}^{\prime} ; H \subset E \subseteq K$. There exists a base $\left\{\theta_{1}, \ldots, \theta_{n}\right\}$ of $E ; 1<a_{k}, 0 \leq a_{j}<a_{k}(1 \leq j \leq k-1)$ such that

$$
\left\{\begin{array}{l}
\theta_{1}=\beta_{\sigma(1)}^{\prime}+a_{1} \beta_{\sigma(k)}^{\prime}, \\
\theta_{2}=\beta_{\sigma(2)}^{\prime}+a_{2} \beta_{\sigma(k)}^{\prime}, \\
\ldots \ldots \ldots \ldots \ldots \ldots \ldots \ldots \\
\theta_{k-1}=\beta_{\sigma(k-1)}^{\prime}+a_{k-1} \beta_{\sigma(k)}^{\prime}, \\
\theta_{k}=a_{k} \beta_{\sigma(k)}^{\prime}, \\
\theta_{k+1}=\beta_{\sigma(k+1)}^{\prime}, \\
\ldots \ldots \ldots \ldots \ldots \ldots \ldots \ldots \ldots \ldots \ldots \ldots \ldots \\
\theta_{n}=\beta_{\sigma(n)}^{\prime} .
\end{array}\right.
$$

Set $\beta_{1}=\beta_{\sigma(1)}^{\prime}, \beta_{2}=\beta_{\sigma(2)}^{\prime}, \ldots, \beta_{n}=\beta_{\sigma(n)}^{\prime}$. Set

$$
\left\{\begin{array}{l}
\gamma_{1}=\gamma_{\sigma(1)}^{\prime}, \\
\gamma_{2}=\gamma_{\sigma(2)}^{\prime} / e_{\sigma(1)}^{\prime}, \\
\gamma_{3}=\gamma_{\sigma(3)}^{\prime} /\left(e_{\sigma(1)}^{\prime} e_{\sigma(2)}^{\prime}\right), \\
\cdots \ldots \ldots \ldots \ldots \ldots \ldots \ldots \ldots \ldots \ldots \ldots \ldots \ldots \ldots \ldots \ldots \\
\gamma_{n}=\gamma_{\sigma(n)}^{\prime} /\left(e_{\sigma(1)}^{\prime} \cdots e_{\sigma(n-1)}^{\prime}\right)
\end{array}\right.
$$


$e_{1}=e_{\sigma(1)}^{\prime}, e_{2}=e_{\sigma(1)}^{\prime} e_{\sigma(2)}^{\prime}, e_{3}=e_{\sigma(1)}^{\prime} e_{\sigma(2)}^{\prime}, e_{\sigma(3)}^{\prime}, \ldots, e_{n}=e_{\sigma(1)}^{\prime} \cdots e_{\sigma(n)}^{\prime} ; F=Z \gamma_{1}+\cdots+Z \gamma_{n}$.

$$
\left\{\begin{array}{l}
\theta_{1}=\beta_{1}+a_{1} \beta_{k}, \\
\theta_{2}=\beta_{2}+a_{2} \beta_{k}, \\
\ldots \ldots \ldots \ldots \ldots \ldots \ldots \\
\theta_{k-1}=\beta_{k-1}+a_{k-1} \beta_{k}, \\
\theta_{k}=a_{k} \beta_{k}, \\
\theta_{k+1}=\beta_{k+1}, \\
\cdots \ldots \ldots \ldots \ldots \ldots \\
\theta_{n}=\beta_{n} .
\end{array}\right.
$$

Suppose $\mathfrak{a}_{H, G}=\mathfrak{a}_{K, G}$. Then, $\mathfrak{a}_{H, G}=\mathfrak{a}_{E, G}=\mathfrak{a}_{K, G} ; \mathfrak{a}_{E, F}=\mathfrak{a}_{K, F}$. For $f_{i} \in A_{F}[X]$, it follows

$$
1-X^{\beta_{k}}=\left(1-X^{\theta_{1}}\right) f_{1}+\cdots+\left(1-X^{\theta_{n}}\right) f_{n} .
$$

Set $X^{y_{i}}=X_{i}$.

$$
\begin{aligned}
1-X_{k}^{e_{k}}= & \left(1-X_{1}^{e_{1}} X_{k}^{a_{1} e_{k}}\right) f_{1}+\cdots+\left(1-X_{k=1}^{e_{k} 1} X_{k}^{a_{k-1} e_{k}}\right) f_{k-1} \\
& +\left(1-X_{k}^{q_{k} e_{k}}\right) f_{k}+\left(1-X_{k+1}^{e_{k}+1}\right) f_{k+1}+\cdots+\left(1-X_{n}^{e_{n}}\right) f_{n} .
\end{aligned}
$$

There are a monomial $M$ of $X_{1}, \ldots, X_{n}$ and $F_{i} \in A\left[X_{1}, \ldots, X_{n}\right]$ such that

$$
\begin{aligned}
M\left(1-X_{k}^{e_{k}}\right)= & \left(1-X_{1}^{e_{1}} X_{k}^{a_{1} e_{k}}\right) F_{1}+\cdots+\left(1-X_{k=1}^{e_{k-1}} X_{k}^{a_{k-1} e_{k}}\right) F_{k-1} \\
& +\left(1-X_{k}^{a_{k} e_{k}}\right) F_{k}+\left(1-X_{k+1}^{e_{k+1}}\right) F_{k+1}+\cdots+\left(1-X_{n}^{e_{n}}\right) F_{n} .
\end{aligned}
$$

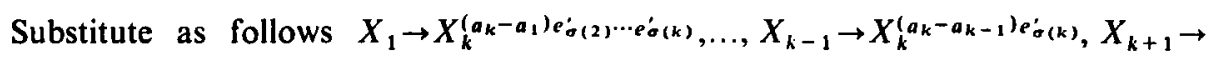
$1, \ldots, X_{n} \rightarrow 1$. Then, we have

$$
X_{k}^{a}\left(1-X_{k}^{e_{k}}\right)=\left(1-X_{k}^{a_{k} e_{k}}\right) F ; 0 \leq a, F \in A\left[X_{k}\right] .
$$

Since $a_{k}>1$, this is obviously impossible.

Proposition 10. If $A_{G}[X]$ is r-noetherian, then $G$ is finitely generated.

Proof. By Prop. 6, the torsion-free rank $n$ of $G$ is finite. Let $\left\{\alpha_{1}, \ldots, \alpha_{n}\right\}$ be independent. $G_{0}=Z \alpha_{1}+\cdots+Z \alpha_{n}$. If $G$ is not finitely generated, there are finitely generated subgroups $G_{i}$ of $G$ such that $G_{0} \subsetneq G_{1} \subsetneq G_{2} \subsetneq \cdots$ and $G=\bigcup_{0}^{\infty} G_{l}$. $G_{l}=Z \alpha_{i 1}+\cdots+Z \alpha_{i n}, \mathfrak{I}_{i}=\left(1-X^{\alpha_{11}}, \ldots, 1-X^{\alpha_{i n}}\right) . \quad$ By Lem. 8, $\mathfrak{I}_{i} \subset \mathfrak{A}_{i+1} . \quad$ Suppose $\mathfrak{A}_{i}=\mathfrak{A}_{i+1}$.

$$
\left\{\begin{array}{l}
1-X^{\alpha_{1+1,1}}=\left(1-X^{\alpha_{11}}\right) f_{11}+\cdots+\left(1-X^{\alpha_{1 n}}\right) f_{1 n}, \\
\cdots \cdots \cdots \cdots \cdots \cdots \cdots \cdots \cdots \cdots \cdots \cdots \cdots \cdots \cdots \cdots \cdots \cdots \cdots \cdots \cdots \cdots \cdots \cdots \cdots \cdots \cdots \cdots \cdots \\
1-X^{a_{1+1, n}}=\left(1-X^{\alpha_{11}}\right) f_{n 1}+\cdots+\left(1-X^{\alpha_{1 n}}\right) f_{n n},
\end{array}\right.
$$


for $f_{j h} \in A_{G}[X]$. There exists $k$ such that $G_{i}, G_{i+1} \subset G_{k}, f_{j h} \in A_{G_{k}}[X]$. This is a contradiction by Lem. 9 . Hence, $\mathfrak{\mathcal { H }}_{i} \subsetneq \mathfrak{P}_{i+1}$. This is a contradiction.

Theorem 11. $A_{G}[X]$ is r-noetherian if and only if $A$ is noetherian and $G$ is finitely generated.

Proof. For ideals $\mathfrak{a} \subsetneq \mathfrak{b}$ of $A,\left(\mathfrak{a}, 1-X^{\alpha}\right) A_{G}[X] \subsetneq\left(\mathfrak{b}, 1-X^{\alpha}\right) A_{G}[X]$ (for fixed $\alpha \neq 0$ ). The rest follows from Prop. 10.

\section{§12. The Dedekind}

The first is on the $\pi$-ring ([13] p. 572).

Proposition 1. If $D_{G}[X]$ is a $\pi$-domain, then so is $D$.

Proof. We confer [13] (46.7). By [24] (3.3), D becomes a Krull domain. Let $p$ be a ht 1 prime of $D$. By [24] (5.1), p $D_{G}[X]$ is a ht 1 prime of $D_{G}[X]$. Hence, $\mathfrak{p}\left(D_{G}[X]: \mathfrak{p} D_{G}[X]\right)=D_{G}[X] . \quad 1=\sum_{1}^{n} p_{i} F_{i}, p_{i} \in \mathfrak{p}, F_{i} \in\left(D_{G}[X]: \mathfrak{p} D_{G}[X]\right) . \quad$ Take $0 \neq$ $p \in \mathfrak{p} . \quad F_{i}=f_{i} / p, f_{i} \in D_{G}[X]$. Let $a_{i}$ be the coefficient of $f_{i}$ of degree 0 . Then, $p\left(a_{1} / p, \ldots, a_{n} / p\right) D=D$. That is, $p$ is invertible.

Lemma 2. Every idempotent of $A_{G}[X]$ belongs to $A$.

Proof. Let $f=f^{2}$. Let $H$ be the subgroup of $G$ generated by the powers of f. $H=Z \alpha_{1} \oplus \cdots \oplus Z \alpha_{n} . \quad X^{\alpha_{i}}=X_{i} . \quad f$ is an idempotent of $A_{Z}\left[X_{1}, \ldots, X_{n}\right] . \quad$ By [32] (3.1), $f \in A_{Z}\left[X_{1}, \ldots, X_{n-1}\right]$. Like this, $f \in A$.

Proposition 3. If $A_{G}[X]$ is a $\pi$-ring, then so is $A$.

Proof. $\quad A_{G}[X]=B_{1} \oplus \cdots \oplus B_{n} \oplus B_{n+1} \oplus \cdots \oplus B_{n+m}, n \geq 0, m \geq 0 ; B_{1}, \ldots, B_{n}$ are $\pi$-domains; $B_{n+1}, \ldots, B_{n+m}$ are special primary rings $([13](46.11)) . \quad 1=e_{1}+\cdots+$ $e_{n+m}, e_{i} \in B_{i} . \quad$ By Lem. $2, e_{i} \in A . \quad B_{i}=\left(e_{i} A\right)_{G}[X] . \quad\left(e_{n+m} A\right)_{G}[X]=B_{n+m}$ is a local ring. This is a contradiction. Hence, $m=0$. By Prop. $1, e_{1} A, \ldots, e_{n} A$ are $\pi$ domains. $A=e_{1} A \oplus \cdots \oplus e_{n} A$. Hence, $A$ is a $\pi$-ring.

THEOREM 4. $A_{G}[X]$ is a $\pi$-ring if and only if $A=\mathfrak{a}_{1} \oplus \cdots \oplus \mathfrak{a}_{n},\left(\mathfrak{a}_{i}\right)_{G}[X]$ is a $\pi$-domain.

Proof. The necessity follows from the proof of Prop. 3. The sufficiency. [13] (46.11).

Remark 5. Even if $A$ is a $\pi$-ring, $A_{2}[X]$ need not a $\pi$-ring.

A Counter Example. $A=Q[x], x^{2}=0 . \quad$ If $A_{Z}[X]$ is a $\pi$-ring, in Th. 4 , $1=e_{1}+\cdots+e_{n}, e_{i} \in a_{i}$. Since $e_{i}$ is an idempotent, $e_{i}=1$. Hence, $n=1$. $A_{Z}[X]$ becomes a $\pi$-domain. This is a contradiction. 
Proposition 6. If $A_{G}[X]$ is a $\pi-r i n g$, then $G$ is of type $(0,0,0, \ldots)$.

Proof. In the proof of Prop. 3, $\left(e_{i} A\right)_{G}[X]$ is a $\pi$-domain. By [13] (46.7), $\left(e_{i} A\right)_{G}[X]$ is a Krull domain. By $[24], G$ is of type $(0,0,0, \ldots)$.

The next is on the Dedekind ring [18].

Proposition 7. If $A_{G}[X]$ is a Dedekind ring, then so is $A$.

Proposition 8. $A_{G}[X]$ is a Dedekind ring if and only if $A_{G}[X]$ is a Krull ring and a prüfer ring.

Lemma 9. Let $(A, \mathfrak{p})$ be a local ring, $\mathfrak{P}=\left(\mathfrak{p}, 1-X^{\alpha} ; \alpha \in G\right)$. If $A_{G}[X]_{\mathfrak{B}}$ is. a Dedekind ring, then so is $A$.

Proof. Let a be a regular ideal. Then $a A_{G}[X]_{\mathfrak{B}}$ is a regular ideal of $A_{G}[X]_{\mathfrak{B}}$. $\mathfrak{n} A_{G}[X]_{\mathbb{F}}=\left(f_{1}, \ldots, f_{n}\right) A_{G}[X]_{\mathfrak{B}}, f_{i} \in A_{G}[X]$. The image $f_{i}(1)$ of $f$ under the canonical homomorphism $A_{G}[X] \rightarrow A$ belongs to $a . \quad a=\left(f_{1}(1) \ldots, f_{n}(1)\right) A$ is finitely generated. Hence, $A$ satisfies the ascending chain condition on regular ideals. Next, let a be a finitely generated regular ideal of $A$. Set $\mathfrak{B}=\left(A_{G}[X]_{\mathfrak{B}}: \mathfrak{a} A_{G}[X]_{\mathfrak{B}}\right)$. Then, $\mathfrak{a} A_{G}[X]_{\mathfrak{P}} \cdot \mathfrak{B}=A_{G}[X]_{\mathfrak{p}}$. Let $a \in \mathfrak{a}$ be a regular element of $A$. $\mathfrak{a} A_{G}[X]_{\mathfrak{\beta}} \cdot\left(h_{1}, \ldots\right.$ $\left.h_{n}\right) A_{G}[X]_{\mathfrak{Y}}=a A_{G}[X]_{\mathfrak{P}}$ for $h_{i} \in A_{G}[X]$. There exists the canonical homomorphism $0: A_{G}[X]_{\mathcal{B}} \rightarrow A$. Then, $\mathrm{b}=\theta\left(\left(h_{1}, \ldots, h_{n}\right) A_{G}[X]_{\mathfrak{k}}\right)$ is a regular ideal of $A . \quad \mathfrak{a b}=a A$. Hence, $\boldsymbol{a}$ is invertible.

The next is on the almost Dedekind rings [22] (2.15).

Proposition 10. If $A_{G}[X]$ is an almost Dedekind ring, then so is $A$.

Proof. Let $\mathfrak{p}$ be a regular maximal ideal of $A . \quad \mathbb{P}=\left(\mathfrak{p}, 1-X^{\alpha} ; x \in G\right) A_{G}[X]$ is a regular maximal ideal of $A_{G}[X]$. Hence, $A_{G}[X]_{\mathfrak{B}}$ is a Dedekind ring. Set $\mathfrak{P}^{\prime}=\left(\mathfrak{p}_{p}, 1-X^{\alpha} ; \alpha \in G\right)\left(A_{\mathfrak{p}}\right)_{G}[X]$. Then, $A_{G}[X]_{\mathfrak{B}} \cong\left(A_{p}\right)_{G}[X]_{\mathfrak{F}}$. By Lem. $9, A_{p}$ is a Dedekind ring.

\section{§13. The rational functions rings}

Proposition 1. If $D_{G}(X)_{\mathrm{loc}}$ is a Dedekind domain, then so is $D$.

Proof. By the proof of $\S 2$, Prop. 2, $D$ is integrally closed. By [26] $\S 4$, Th. 10, $D$ is an almost Dedekind domain. Let $0 \neq d \in D$. Let in be a maximal ideal of $D$ containing $d . \quad m D_{G}(X)_{\text {loc }}$ is a maximal ideal of $D_{G}(X)_{\text {loc }}$ containing $d$ ([26] $\S 7$, Prop. 6). And $m D_{G}(X)_{\text {loc }} \cap D=\mathfrak{m}$ ([26] $\$ 7$, Prop. 2). Hence, $d$ belongs to only finitely many maximal ideals of $D$. Hence, $D$ is a Dedekind domain.

Remark 2. Even if $D$ is a Dedekind domain, $C\left(D_{Z}(X)_{\text {loc }}\right)$ need not be isomorphic with $C(D)$. 
A Counter Example. Let $D$ be a non-factorial Dedekind domain. $D_{\mathrm{Z}}(X)_{\text {loc }}$ is a PID ([21] p. 74, $\left.16(\mathrm{~g})\right)$.

Proposition 3. Let $D$ be a Dedekind domain. Then $D_{G}(X)_{\text {loc }}$ is a PID.

Proof. Let $\mathfrak{A}$ be an ideal of $D_{G}(X)_{\text {loc. }}$ Suppose $\mathfrak{A}$ is not finitely generated. Then, $\left(\xi_{1}\right) \subsetneq\left(\xi_{1}, \xi_{2}\right) \subsetneq\left(\xi_{1}, \xi_{2}, \xi_{3}\right) \subsetneq \cdots \subset \mathfrak{A}$. By [26] $\$ 7$, Prop. 7(2), $D_{G}(X)_{\text {loc }}$ is bezout. Hence, $\left(\xi_{1}, \ldots, \xi_{i}\right)=f_{i} D_{G}(X)_{\text {loc }}$ for $f_{t} \in D_{G}[X] . \quad f_{i}=f_{i+1}(h / g), h / g \in$ $D_{G}(X)_{\text {loc }}$. By [26] $\$ 2$, Th. 11, $A_{f_{i}}=A_{f_{i}} A_{g}=A_{f_{i g}}=A_{f_{i+1} h}=A_{f_{i+1}} A_{h h}$. Since $h / g$ is non-unit of $D_{G}(X)_{\mathrm{loc}}, A_{f_{1+1}} A_{h} \subsetneq A_{f_{1+1}}$. Hence, $A_{f_{1}} \subsetneq A_{f_{2}} \subsetneq \cdots$. A contradiction. Hence, $D_{G}(X)_{\text {loc }}$ is noetherian. By [26] $\S 7$, Prop. $7(2), D_{G}(X)_{\text {loc }}$ is a PID.

Proposition 4. Assume $D$ be a local domain such that $D_{G}(X)_{10 c}$ is factorial. Then, $D$ is factorial.

Proof. (1) $D$ is integrally closed. Let $E$ be the integral closure of $D$. By [26] $\$ 6$, Prop. 9, $E_{G}(X)_{\text {loc }}$ is integral over $D_{G}(X)_{\text {loc }}$. Hence, $E_{G}(X)_{\text {loc }}=D_{G}(X)_{\text {loc }}$. Hence, $E=D$. (2) Every nonunit of $D$ is a nonunit of $D_{G}(X)_{\text {loc }}$ (3) Every element of $D$ is a finite product of irreducible elements of $D$. (4) Every irreducible element of $D$ is irreducible in $D_{G}(X)_{\text {loc }}$. Let $a$ be an irreducible element of $D$. Let $a=$ $(f / g)\left(f^{\prime} / g^{\prime}\right)$ be a decomposition in $D_{G}(X)_{\text {loc }}$. Since $A_{f f^{\prime}}=a D, A_{f}, A_{f^{\prime}}$ are principal ideals of $D$ ([26] §6, Prop. 12). Set $A_{f}=b D, A_{f^{\prime}}=b^{\prime} D$. By [26] §6, Prop. 1 (4), $b b^{\prime} D=A_{f} A_{f^{\prime}}=A_{f f^{\prime}}=a D . \quad a=b b^{\prime} c$ for $c \in D$. Since $a$ is irreducible in $D$, $A_{f}=D$ or $A_{f^{\prime}}=D$. Hence, $f / g$ or $f^{\prime} / g^{\prime}$ is a unit of $D_{G}(X)_{\text {loc }}$. (5) If $a, b \in D$ are associated in $D_{G}(X)_{\text {loc }}$, then they are associated in $D$. (6) $D$ is factorial.

Proposition 5. Assume $A$ be a regular ring such that $A_{G}(X)_{\text {loc }}$ is noetherian local. Then, $A_{G}(X)_{\text {loc }}$ is a regular local ring.

Proof. By [26] §7, Prop. 6, $A$ is local. By [26] $\$ 7$, Prop. 2, $A$ is noetherian. Set $\operatorname{dim} A=d ; \operatorname{dim} A_{G}(X)_{\mathrm{loc}} \geq d$. The maximal ideal $\mathrm{m}$ of $A$ is generated by $d$ elements $x_{1}, \ldots, x_{d} . \quad \mathfrak{m} A_{G}(X)_{\text {loc }}=\left(x_{1}, \ldots, x_{d}\right) A_{G}(X)_{\text {loc }}$. Hence, $d \geq$ $\operatorname{dim} A_{G}(X)_{\text {loc. }}$ Hence, $\operatorname{dim} A_{G}(X)_{\text {loc }}=d$.

Proposition 6. Assume $R_{G}(X)_{\text {loc }}$ is noetherian local for a ring $R$. Then the multiplicity of $R_{G}(X)_{\mathrm{loc}}$ is that of $R$.

Proof. By [26] $\$ 7$, Prop. 2, Prop. 6, $R$ is noetherian local. Let $m$ be the maximal ideal of $R, R^{*}=R_{G}(X)_{\text {loc }}$ By [26] $\$ 7$, Prop. $1, R^{*}$ is a faithfully flat $R$-module. Set length $R / m\left(\mathrm{~m}^{i} / \mathrm{m}^{i+1}\right)=l$.

$$
\begin{aligned}
& \left(\mathrm{m}^{i} R^{*}\right) /\left(\mathrm{m}^{i+1} R^{*}\right) \cong\left(\mathrm{m}^{i} / \mathrm{m}^{i+1}\right) \otimes_{R} R^{*} \cong(\underbrace{R / \mathrm{m} \oplus \cdots \oplus R / \mathrm{m})}) \\
& \otimes_{R} R^{*} \cong \underbrace{R^{*} / \mathrm{m} R^{*} \oplus \cdots \oplus R^{*} / \mathrm{m} R^{*}} .
\end{aligned}
$$

Hence, length $R^{*} / m R^{*}\left(m^{i} R^{*}\right) /\left(m^{i+1} R^{*}\right)=$ length ${ }_{R / m^{1}} n^{i} / m^{i+1}$. Hence, 


$$
\text { length } R^{*} R^{*} / \mathrm{mi}^{i+1} R^{*}=\text { length } R / \mathrm{m}^{i+1} \text {. }
$$

Proposition 7. Let $(A, \mathrm{~m})$ be a noetherian local ring. Then, $\bigcap_{0}^{\infty} \mathrm{m}^{i} A_{\mathrm{G}}(X)_{\mathrm{loc}}=0$.

Proof. Let $f / g \in \cap \mathrm{m}_{i} A_{G}(X)_{\text {loc }}$. Let $H$ be the subgroup of $G$ generated by the powers of $f, g$. By [7] Lem. $1, A_{G}[X]$ is a faithfully flat $A_{H}[X]$-module. Hence, $A_{G}(X)_{\text {loc }}$ is a flat $A_{H}(X)_{\text {loc }}$-module $([19](0,6.3 .2)) . A_{H}(X)_{\mathrm{loc}} \subseteq A_{G}(X)_{\mathrm{loc}}$. By [29] (18.3), $\mathrm{m}^{i} A_{\mathrm{G}}(X)_{\mathrm{loc}} \cap A_{H}(X)_{\mathrm{loc}}=\mathrm{m}^{i} A_{H}(X)_{\mathrm{loc}}$. Hence $f / g \in \mathrm{m}^{i} A_{H}(X)_{\mathrm{loc}}$ for all $i$. Since $A_{H}(X)_{\text {loc }}$ is noetherian, $\cap \mathrm{m}^{i} A_{H}(X)_{\mathrm{loc}}=0$. Hence, $f / g=0$.

Remark 8. Even if $A$ and $A_{G}(X)_{\text {loc }}$ are noetherian local, $G$ need not be of finite torsion-free rank.

A Counter Example. $\quad A=k$ a field, $G=\underbrace{Z \oplus \cdots}_{\mathbb{N}_{0}}$.

Proposition 9. Let $A$ be a noetherian local ring. Assume $\operatorname{dim} A_{G}(X)_{\text {loc }}$ $=\operatorname{dim} A$ for every free $G$, then $\operatorname{dim} A_{G}(X)_{\text {loc }}=\operatorname{dim} A$ for any $G$.

Proof. Let $\left\{\alpha_{\lambda} ; \Lambda\right\}$ be a maximal independent subset of $G . \quad K=\sum_{\lambda} Z \alpha_{\lambda}$. $\operatorname{dim} A_{K}(X)_{\text {loc }}=\operatorname{dim} A=n$. ht p $A_{G}[X] \leq \mathrm{htp} A_{K}[X]=n$. Hence, $\operatorname{dim} A_{\mathrm{G}}\left(X^{\lambda}\right)_{\text {loc }}=$ $\operatorname{dim} A$.

\section{\$14. The others}

Proposition 1. Let $\mathfrak{q}$ be a p-primary in $A$. Then, $\mathfrak{q} A_{G}[X]$ is $\mathfrak{p} A_{G}[X]-$ primary in $A_{G}[X]$.

Proof. Let $f, g \in A_{G}[X], f g \in \mathfrak{q}^{e}=\mathfrak{q} A_{G}[X], g \bar{\epsilon} \mathfrak{q}^{e}$. We may suppose $\mathfrak{q}=0$. Since $f$ is a zerodivisor of $A_{G}[X], a f=0$ for $0 \neq a \in A$ ([26] $\$ 9$, Prop. 5). $f=$ $\sum a_{\alpha} X^{\alpha} . \quad a_{\alpha} a=0 . \quad a_{\alpha} \in \mathfrak{p} . \quad$ Hence, $f \in \mathfrak{p}^{e}=\mathfrak{p} A_{G}[X]$.

The next is on the primitive rings [31] $\S 12$.

RemarK 2. $A_{G}[X]$ is never a primitive ring.

Proof. [31] (32.3).

THEOREM 3. $A_{G}[X]$ is arithmetical if and only if $A$ is von Neumann regular, $G \subset Q$.

Proof. The necessity. By [13] p. 321, 20, $A_{G}[X]$ is a prüfer ring. By [16] (3.1), $A$ is von Neumann regular, $G \subseteq Q$. The sufficiency. Let $M$ be a maximal ideal of $A_{\mathfrak{G}}[X], M \cap A=\mathfrak{p}, A \mid \mathfrak{p}=S . \quad \mathfrak{B}=M_{S}$ is a maximal ideal of $\left(A_{\mathfrak{p}}\right)_{G}[X]$ $=A_{\mathfrak{G}}[X]_{s} . \quad A_{\mathfrak{p}}=k$ is a field. Let $0 \neq f, g \in \mathfrak{P} . \quad$ Let $H$ be the subgroup generated by the powers of $f, g . \quad H=Z \alpha$. Set $\mathfrak{B} \cap k_{H}[X]=\mathfrak{P}^{\prime} . \quad k_{H}[X]_{\mathfrak{B}^{\prime}}$ is a discrete valua- 
tion ring. Hence, $f$ divides $g$ or $g$ divides $f$ in $k_{H}[X]_{\mathfrak{B}^{\prime}}$. Assume $f$ divides $g$. Then, $g \in f k_{G}[X]_{\mathfrak{B}} . \quad k_{G}[X]_{\mathfrak{F}}$ is a chained ring $\left([13]\right.$ p. 184,8). Since $A_{G}[X]_{M}$ $=k_{G}[X]_{\mathfrak{R}}, A_{G}[X]$ is an arithmetical ring $([13]$ p. 321,19$)$.

REMARK 4. Even if $f$ is a unit of $A_{G}[X]$, where $G$ is totally ordered, the leading coefficient need not be a unit of $A$.

We confer [25] (1.1).

Proposition 5. If $A_{G}[X]$ is a multiplication ring, then $A$ is von Neumann regular and $G \cong Z$.

Proof. [16] (3.1).

Proposition 6. If $A_{G}[X]$ is a multiplication ring, then so is $A$.

Proof. Let $\mathfrak{b} \subset \mathfrak{a}$ be ideals of $A . \quad b A_{G}[X]=\mathfrak{a} A_{G}[X] \cdot \mathfrak{B}$ for an ideal $\mathfrak{B}$ of $A_{\mathrm{G}}[X]$. Let $\mathrm{c}$ be the set of coefficients of elements of $\mathfrak{B}$ of degree 0 . Then, $\mathfrak{b}=$ ac.

THEOREM 7. $A_{G}[X]$ is a multiplication ring if and only if $A$ is a finite direct sum of fields and $G \cong Z$.

Proof. The necessity. By Prop. 5, $A$ is von Neumann regular and $G \cong Z$. Let $\mathfrak{p}$ be a prime ideal of $A . \quad \mathfrak{B}=(\mathfrak{p}, 1-X)$ is a ht 1 prime of $A_{Z}[X]$. Hence, $\mathfrak{P}$ is a finitely generated ideal (cf. the proof of [3] $3(6.1)$ ). $\quad \mathbb{P}=\left(f_{1}, \ldots, f_{n}\right)$ for $f_{i} \in$ $A_{Z}[X] . \quad f_{i}(1) \in \mathfrak{p} . \quad$ Let $a \in \mathfrak{p} . \quad a=\sum f_{i} g_{i}, g_{i} \in A_{Z}[X] . \quad a=\sum f_{i}(1) g_{i}(1) . \quad p=\left(f_{1}(1)\right.$, $\left.\ldots, f_{n}(1)\right)$. Hence, $A$ is noetherian.

The next is on the QQR-property ([13] p. 339, 2).

Proposition 8. $D_{G}[X]$ has $Q Q R$-property if and only if $D$ is a field and $G \hookrightarrow Q$.

Proof. The necessity. Let $E$ be the integral closure of $D . E_{G}[X]$ has also the QQR-property. By $[24](4.4), E_{G}[X]$ is integrally closed. By [13] p. 340, $4, E_{G}[X]$ is prüfer. By [16], $E$ is a field and $G G Q$. The sufficiency is similar.

Proposition 9. (1) If $A_{G}[X]$ is lasker, then so is $A$. (2) If $A_{G}[X]$ is strongly lasker, then so is $A$.

Remark 10. Even if $k$ is a field, $G$ of type $(0,0,0, \ldots), k_{G}[X]$ need not be lasker.

$\Lambda$ Counter Example. $G=Z \oplus Z \oplus \cdots, \quad k_{G}[X]=k_{Z}\left[X_{1}, X_{2}, X_{3}, \ldots\right]=D$. $M_{1}=\left\{1-X_{1}\right\}, M_{2}=\left\{1-X_{2}, 1-X_{3}\right\}, M_{3}=\left\{1-X_{4}, \ldots, 1-X_{7}\right\}, \ldots . \quad$ I $=\left(1-X_{1}\right.$, $\left.\left(1-X_{2}\right)\left(1-X_{3}\right),\left(1-X_{4}\right) \cdots\left(1-X_{7}\right),\left(1-X_{8}\right) \cdots\left(1-X_{15}\right), \ldots\right)$. Take $g_{i} \in M_{i}$, let $\mathfrak{P}=\left(g_{1}, g_{2}, g_{3}, \ldots\right)$. $\mathfrak{P}$ is a prime ideal containing $\mathfrak{T}$. $\mathfrak{P}$ is a minimal prime ideal 
containing $\mathfrak{H}$. Such $\mathfrak{B}$ exist infinitely many. Hence, $\mathscr{H}$ cannot be a finite intersection of primary ideals.

Example 11. $D=k\left[X_{1}, X_{2}, X_{3}, \ldots\right]$ is not lasker.

Proposition 12. If $D_{G}[X]$ has the $Q R$-property, so does $D$.

Proposition 13. $D_{G}[X]$ has the QR-property if and only if $D$ is a field and $G G Q$.

Proof. The necessity. $D_{G}[X]$ is a prüfer domain. By $[16], D$ is a field and $G \hookrightarrow Q$. The sufficiency. By $[16], D_{G}[X]$ is bezout. By $[13](27.5), D_{G}[X]$ has the QR-property.

If every overring $E$ of $D$ (i.e. $D \subset E \subset K$ ) is a finite intersection of quotient rings of $D, D$ is said to have FQR-property.

Proposition 14. $D_{G}[X]$ has the FQR-property if and only if $D_{G}[X]$ has the $Q R$-property.

Proof. Prop. 13, Prop. 8.

$=\left({ }_{1}\right.$, The next is on the irreducible rings ([31] p. 61).

Proposition 15. If $A_{G}[X]$ is irreducible, then sa is $A$.

The next is on the root closed ([13] p. 173).

Proposition 16. If $A_{G}[X]$ is root closed, then so is $A$.

Remark 17. Even if $A$ is root closed, $A_{Z}[X]$ need not be so.

A Counter Example. $A=Z /(4) . \quad(2 /(1+X))^{2} \in A_{Z}[X]$.

Remark 18. Fix $G$. Assume $D$ be a domain of transcendental degree I over a field. There is not a field such that $D \cong k_{G}[X]$. Then $D$ need not be $G$ strongly invariant.

A ring $A$ is said to be $G$-strongly invariant provided whenever $\sigma: A_{G}\left[X_{1}, \ldots\right.$, $\left.X_{n}\right] \cong B_{G}\left[Y_{1}, \ldots, Y_{n}\right]$ is an isomorphism, then $\sigma(A)=B$.

A Counter Example. $D=Q_{Z}[x], x$ an indeterminate. $G=Z \oplus Z$.

Remark 19. Assume $\boldsymbol{A}$ is a Dedekind domain which is not $G$-strongly invariant. Then, there need not exist $s \in A$ and a field $k$ such that $A[1 / s]=k_{G}[X]$.

A Counter Example. $A=Q_{Z}[x], x$ an indeterminate. $G=Z \oplus Z$. If $A[1 / s]=k_{Q}[X], 1=$ transc. $\quad \operatorname{degree}_{Q} A[1 / s]=$ transc. degree $_{Q} k_{G}[X] \geq 2$. A contradiction.

The next is on the strongly invariant [11] $(0.3)$. 
Proposition 20. If $A_{G}[X]$ is strongly invariant, then so is $A$.

Proof. Let $B$ be a ring and $\sigma: A\left[X_{1}, \ldots, X_{n}\right] \cong B\left[Y_{1}, \ldots, Y_{n}\right]$. We get a natural homomorphism $\sigma^{\prime}:\left(A\left[X_{1}, \ldots, X_{n}\right]\right)_{G}[X] \cong\left(B\left[Y_{1}, \ldots, Y_{n}\right]\right)_{G}[X]$. Hence, $\left(A_{G}[X]\right)\left[X_{1}, \ldots, X_{n}\right] \cong\left(B_{G}[X]\right)\left[Y_{1}, \ldots, Y_{n}\right]$. Hence, $\sigma^{\prime}\left(A_{G}[X]\right)=B_{G}[X]$. Hence, $\sigma(A)=B$.

The next is on the coherence [4] $\S 2$, exerc. 12) $\alpha$ ).

Proposition 21. If $A_{G}[X]$ is coherent, then so is $A$.

Proof. $A_{G}[X]$ is a faithfully flat $A$-module ([26] $\S 9$, Prop. 1). Let a be any cardinal number. The direct product $\Pi A_{G}[X]$ of the number $\mathfrak{a}$ of $A_{G}[X]$ is flat as $A$-module (e.g. [27] (3.B)). Let $\left(a_{n}^{1}\right)_{n \in a},\left(a_{n}^{2}\right)_{n \in a}, \ldots,\left(a_{n}^{m}\right)_{n \in a}$ be a finite number of elements of $\prod_{a} A ; a_{1}, \ldots, a_{m} \in A$ such that

$$
a_{1}\left(a_{n}^{1}\right)+\cdots+a_{m}\left(a_{n}^{m}\right)=(0) .
$$

By [4] I, § 2, Cor. 1 of Prop. 13,

$$
\left(a_{n}^{i}\right)=a_{1 i}\left(f_{n}^{1}\right)+\cdots+a_{l i}\left(f_{n}^{l}\right), \quad \sum a_{j i} a_{i}=0,
$$

$\left(f_{n}^{j}\right) \in \prod A_{G}[X], a_{j i} \in A$. Let $b_{n}^{j}$ be the constant term of $f_{n}^{i}$. Then, $\left(a_{n}^{i}\right)=$ $\sum a_{j i}\left(b_{n}^{j}\right)$. Hence, $\prod_{a} A$ is a flat $A$-module.

The next is on the hereditary [8] I, §5.

Proposition 22. If $A_{G}[X]$ is hereditary, then so is $A$.

Proof. Set $\mathfrak{N}=\left(1-X^{\alpha} ; \alpha \in G\right) A_{\mathrm{G}}[X]$. We have a natural onto homomorphism $\gamma: A_{G}[X] \rightarrow A$. Let $\mathfrak{a}$ be an ideal of $A ; Q^{\prime}, Q^{\prime \prime}$ be $A$-modules; $\alpha: \mathfrak{a} \rightarrow Q^{\prime \prime}$ an $A$-homomorphism; $\beta: Q^{\prime} \rightarrow Q^{\prime \prime}$ an onto $A$-homomorphism. $Q^{\prime}, Q^{\prime \prime}$ become $A_{G}[X]$-modules. $\beta$ is an $A_{G}[X]$-homomorphism. $\alpha$ is also an $A_{G}[X]$-homomorphism. There exists an $A_{G}[X]$-homomorphism $\gamma \mid \mathfrak{a} A_{G}[X]: \mathfrak{a} A_{G}[X] \rightarrow \mathfrak{a}$. Hence, there exists an $A_{G}[X]$-homomorphism $\delta: \mathfrak{a} A_{G}[X] \rightarrow Q^{\prime}$ such that $\beta \delta=\alpha\left(\gamma \mid \mathfrak{a} A_{G}[X]\right)$. $\beta(\delta \mid \mathfrak{a})=\alpha . \quad \delta \mid \mathfrak{a}$ is an $A$-homomorphism. Hence, $\mathfrak{a}$ is a projective $A$-module.

Proposition 23. If $A_{G}[X]$ is semi-hereditary, then so is $A$.

Proof. Similar with that of Prop. 22.

Proposition 24. $A_{G}[X]$ is semi-hereditary if and only if $A$ is von Neumann regular and $G G Q$.

Proof. The necessity. By [17] Th. 20, $A_{G}[X]$ is prüfer. By [16], $A$ is von Neumann regular and $G \hookrightarrow Q$. The sufficiency. $A_{G}[X]$ is a prüfer ring and the nilradical of $A$ is zero. By [26] $\S 3$, Th. 3, the nilradical of $A_{G}[X]$ is also zero. Hence, the nilradical of $A_{G}(X)$ is also zero. Hence, $A_{G}(X)$ is von Neumann regular. 
The next is on the condition $(M)([40]$ p. 15$)$.

Proposition 25. $D_{G}[X]$ satisfies the $(M)$ if and only if $D$ satisfies $(M)$ and $G$ is of type $(0,0,0, \ldots)$.

Proof. The necessity. Let $a_{i} \in D, a_{i} D \subset a_{i+1} D, i=1,2,3, \ldots$. There exists $n$ such that $a_{i} D_{G}[X]=a_{i+1} D_{G}[X]$ for all $i \geq n$. It follows $a_{i} D=a_{i+1} D$ for all $i \geq n$. As the proof of $[24](2.3), G$ is seen of type $(0,0,0, \ldots)$. The sufficiency. Let $0 \neq f_{i} \in D_{G}[X], f_{i} D_{G}[X] \subset f_{i+1} D_{G}[X], i=1,2,3, \ldots, K_{G}[X]$ is a Krull domain ([24](3.3)). Hence, there exists $n$ such that $f_{i} K_{G}[X]=f_{i+1} K_{G}[X]$ for all $i \geq n$. We may suppose $n=1$. It follows $f_{i}=f_{i+1} a_{i}, a_{i} \in D$. Set $o\left(f_{1}\right)=\alpha$ and let $c_{i}$ be the coefficient of $X^{\alpha}$ in $f_{i} \cdot \quad c_{i} D \subset c_{i+1} D$. There exists $m$ such that $c_{i} D=c_{i+1} D$ for all $i \geq m$. Hence, $a_{i}$ is a unit for any $i \geq m$. Hence, $f_{i} D_{G}[X]=f_{i+1} D_{G}[X]$ for all $i \geq m$.

Proposition 26. If $A_{G}[X]$ satisfies $(M)$, then so does $A$ and $G$ is of type $(0,0,0, \ldots)$.

Proof. Let $a_{1}, a_{2}, \ldots \in A, a_{i} A \subset a_{i+1} A$ for $i=1,2,3, \ldots$ There exists $n$ such that $a_{i} A_{G}[X]=a_{i+1} A_{G}[X]$ for all $i \geq n$. $a_{i+1}=a_{i} b_{i}, b_{i} \in A$ for all $i \geq n$. Hence, $a_{i} A=a_{i+1} A$ for all $i \geq n$. Hence, $A$ satisfies $(M)$. For a prime number $p$ and $0 \neq \alpha \in G$, suppose $p^{n+1} \alpha_{n+1}=\alpha$ is solvable. $\left(1-X^{\alpha_{n-1}}\right) \subsetneq\left(1-X^{\alpha_{n}}\right)$. Hence, $A_{G}[X]$ does not satisfy $(M)$. The rest is similar.

The next is on the $M$-ring [36].

Proposition 27. $D_{G}[X]$ is a $M$-ring if and only if $D$ is a field and $G \cong Z$.

Proof. The necessity. By [36] 2, Th. 1, it follows $\operatorname{dim} D_{G}[X] \leq 1$. If $D$ is not a field, take a maximal ideal $\mathfrak{p}$ of $D$. Since $D_{G}[X] / \mathfrak{p} D_{G}[X] \cong(D / \mathfrak{p})_{G}[X]$, $\mathfrak{p} D_{G}[X]$ is not a maximal ideal of $D_{G}[X]$. Hence, $\operatorname{dim} D_{G}[X]>1$. A contradiction. Hence, $D=K$ is a field. By [24] Prop. 4.4, [37] I, Th. 6, $K_{G}[X]$ is a Dedekind domain. By $[16], G \cong Z$. The sufficiency. $K_{Z}[X]$ is an integrally closed Mori-ring. By [36] 2, Cor. of Th. $4, K_{Z}[X]$ is a $M$-ring.

Remark 28. Even if $G$ is free, every homogeneous ideal a of $k_{G}[X, Y]$ need not be a finite intersection of primary ideals.

The homogeneous ideal of $k_{G}[X, Y]$ is an ideal generated by homogeneous elements.

A Counter Example. $G=Z \oplus Z \oplus \ldots . \quad X^{\left(0, \ldots, 0, \frac{1}{i}, 0, \ldots .\right)}=X_{i}, Y^{\left(0, \ldots, 0, \frac{1}{i}, 0, \ldots\right)}$ $=Y_{i} . \quad k_{G}[X, Y]=k_{Z}\left[X_{1}, X_{2}, \ldots ; Y_{1}, Y_{2}, \ldots\right] . \quad$ Set $\mathfrak{N}=\left(X_{1}-Y_{1},\left(X_{2}-Y_{2}\right)\left(X_{3}-\right.\right.$ $\left.\left.Y_{3}\right),\left(X_{4}-Y_{4}\right) \cdots\left(X_{7}-Y_{7}\right),\left(X_{8}-Y_{8}\right) \cdots\left(X_{15}-Y_{15}\right), \ldots\right)$. The fact that $\mathfrak{A}$ is not the finite intersection follows as the proof of Remark 10. 
A ring $A$ is said to be $G$-invariant provided that whenever $A_{G}\left[X_{1}, \ldots, X_{n}\right] \cong$ $B_{G}\left[Y_{1}, \ldots, Y_{n}\right]$ for a ring $B$, then $A \cong B$.

Remark 29. Assume $D$ is a domain of transcendence degree 1 over a field and $G$ be free. Then, $D$ need not be $G$-invariant.

A Counter Example. Let $k$ be a field, $x$ an indeterminate, $D=k_{z}[x]$, $G=\underbrace{Z \oplus Z}_{\aleph_{0}}, E=k$.

\section{References}

[1] Abhyanker, Eakin, Heinzer, On the uniqueness of the coefficient ring in a polynomial ring, J. of Algebra 23 (1972), 310-342.

[2] Akizuki, Nagata, Modern Algebra, Kyoritsu, Tokyo, 1957.

[3] D. Anderson, Multiplication ideals, multiplication rings, and the ring $R(X)$, Canad. J. Math. 28 (1976), 760-768.

[4] N. Bourbaki, Algèbre commutative, chap. 1, 2, Hermann, Paris, 1961.

[5] N. Bourbaki, Algèbre, chap. 6, 7, Hermann, Paris, 1964.

[6] N. Bourbaki, Algèbre commutative, chap. 7, Hermann, Paris, 1965.

[7] Brewer, Costa, Lady, Prime ideals and localization in commutative group rings, J. of Algebra 34 (1975), 300-308.

[8] Cartan, Eilenberg, Homological algebra, Princeton Univ. Press, 1956.

[9] I. Connell, On the group ring, Canad. J. Math. 15 (1963), 650-685.

[10] E. Davis, Overrings of commutative rings, I, Noetherian overrings, Trans. Amer. Math. Soc., 104 (1962), 52-61.

[11] Eakin, Heinzer, A cancellation probrem for rings, Conference on commutative algebra, Springer Lecture note 311, 1973.

[12] R. Fossum, The divisor class group of a Krull domain, Springer-Verlag, Ergeb. Math. 74, 1973.

[13] R. Gilmer, Multiplicative ideal theory, Marcel Dekker, New York, 1972.

[14] R. Gilmer, The $n$-generator property for commutative rings, Proc. Amer. Math. Soc., 38 (1973), 477-482.

[15] Gilmer, Parker, Divisibility properties in semigroup rings, Michigan Math. J. 21 (1974), 65-86.

[16] Gilmer, Parker, Semigroup rings as prüfer rings, Duke Math. J. 41 (1974), 219-230.

[17] M. Griffin, Prüfer rings with zero divisors, J. Reine Angew. Math. 239/240 (1969), 5567.

[18] M. Griffin, Valuation theory and multiplication rings, Queens Univ. Preprint, 1970.

[19] A. Grothendieck, Éléments de géometrie algébrique l, Publ. Math., I. H. E. S., 1960.

[20] A. Grothendieck, Éléments de geometrie algebrique 4-1, Publ. Math., I. H. E. S., 1964.

[21] I. Kaplansky, Commutative rings, Univ. Chicago Press, 1974.

[22] R. Kennedy, A generalization of Krull domains to rings with zero divisors, Thesis, Univ. Missouri Kansas, 1973.

[23] D. Lantz, Preservation of local properties and chain conditions in commutative group rings, Pacific J. Math., 63 (1976), 193-199.

[24] R. Matsuda, On algebraic properties of infinite group rings, Bull, Fac. Sci., Ibaraki Univ. 7 (1975), 29-37.

[25] R. Matsuda, Notes on power series rings, Bull. Fac. Sci., Ibaraki Univ. 7 (1975), 
17-27.

[26] R. Matsuda, Infinite group rings II, Bull. Fac. Sci., Ibaraki Univ. 8 (1976), 43-66.

[27] H. Matsumura, Commutative algebra, Benjamin, New York, 1970.

[28] M. Nagata, A remark on the unique factorization theorem, J. Math. soc. Japan 9 (1957), 143-145.

[29] M. Nagata, Local rings, Interscience, New York, 1962.

[30] M. Nagata, Theory of commutative rings, Kinokuniya, Tokyo, 1974.

[31] Nakayama, Azumaya, Algebra II, Iwanami, Tokyo, 1954.

[32] M. Parmenter, Isomorphic group rings, Canad. Math. Bull. 18 (1975), 567-576.

[33] E. Pirtle, A note on almost Dedekind domains, J. Sci. Hiroshima Univ. 32 (1968), 441447.

[34] E. Pirtle, Families of Valuations and semigroups of fractionary ideal classes, Trans. Amer. Math. Soc., 144 (1969), 427-439.

[35] E. Pirtle, On a generalization of Krull domains, J. of Algebra 14 (1970), 485-492.

[36] J. Querré, Sur les anneaux réflexifs, C. R. Acad. Sc. Paris 279 (1974), 45-47.

[37] N. Raillard, Sur les anneaux de Mori, C. R. Acad. Sc. Paris 280 (1975), 1571-1573.

[38] L. Ratliff, Chain conjectures and H-domains, Conference on Commutative algebra, Springer Lecture note 311, 1973.

[39] L. Ratliff, Four notes on saturated chains of prime ideals, J. of Algebra 39 (1976), 75-93.

[40] P. Samuel, Lectures on unique factorization domains, Tata, 1964.

[41] P. Samuel, About euclidean rings, J. of Algebra 19 (1971), 282-301.

[42] J.-P. Soublin, Anneaux et modules cohérents, J. of Algebra 15 (1970), 455-472.

[43] A. Zaks, Half factorial domains, Bull. Amer. Math. Soc., 82 (1976), 721-723.

[44] Zariski, Samuel, Commutative algebra I, Van Nostrand, Princeton, 1958.

[45] Zariski, Samuel, Commutative algebra II, Van Nostrand, Princeton, 1960. 